\title{
Tin-Free Generation of Alkyl Radicals from Alkyl 4-Pentynyl Sulfides via Homolytic Substitution at the Sulfur Atom
}

\author{
Giorgio Bencivenni, Tommaso Lanza, Rino Leardini, Matteo Minozzi, * Daniele Nanni, * \\ Piero Spagnolo, * and Giuseppe Zanardi \\ Dipartimento di Chimica Organica “A. Mangini”, Università di Bologna, Viale Risorgimento 4, I-40136 Bologna, Italy. \\ minus@ms.fci.unibo.it \\ Supporting information
}

Table of contents:

Page 1: General Remarks and Syntheses of Precursors.

Page 4: Syntheses and Characterizations of Sulfides 1a-i.

Page 6: Reactions of Sulfides 1a-f.

Page 8: Reactions of Sulfides 1g-i.

Page 10: NMR Spectra. 
General Remarks. ${ }^{1} \mathrm{H}$ and ${ }^{13} \mathrm{C}$ NMR spectra were recorded in $\mathrm{CDCl}_{3}$ solutions using tetramethylsilane as internal standard. Coupling constants are given in $\mathrm{Hz}$. IR spectra were recorded in $\mathrm{CHCl}_{3}$ solutions or as liquid films. Mass spectra were usually recorded using a ThermoFisher - Focus DSQ system. Column chromatography was performed on ICN silica gel (63-200, $60 \AA$ ) by gradual elution with $n$ hexane / diethyl ether. All the following chemicals were purchased from Aldrich and used without further purification: 1-bromododecane 11a, 1-(2-bromopropyl)benzene 11b, 1-(bromomethyl)-4methoxybenzene 11d, dodecane-1-thiol 12a, 1-adamantanethiol 12c, (4-methoxyphenyl)methanethiol 12d, 1-(3-chloropropyl)benzene 18a, 1-(3-bromopropyl)benzene 18b, 1-(3-iodopropyl)benzene 18c, 3-phenylpropan-1-ol, 2-bromoacetyl bromide 19, 5-bromopentyl acetate, 5-chloropent-1-yne, thiophenol, 1,1'-azobis(cyclohexanecarbonitrile) (ACCN).

\section{Precursors.}

SH 1-Phenylpropane-2-thiol (12b). ${ }^{1}$ Thiol $\mathbf{1 2 b}$ was prepared according to the previously reported procedure. MS: m/z (rel. inten.) 152 (23), 118 (4), 92 (100), 91 (89), 65 (19), 61 (75).

$\mathrm{TsO}_{\mathrm{Ph}}$ 3-Phenylpropyl 4-methylbenzenesulfonate (18d). ${ }^{2}$ The target compound was prepared starting from 3-phenylpropan-1-ol according to the previously reported procedure. MS: $m / z$ (rel. inten.) 290 (1), 119 (12), 118 (96), 117 (100), 91 (46), 65 (20).

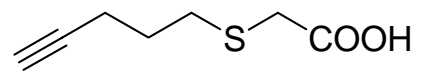

2-(Pent-4-ynylthio)acetic acid (13). Potassium hydroxide (2.2 mmol) was added at $0{ }^{\circ} \mathrm{C}$ to a solution of 2 -mercaptoacetic acid $(1 \mathrm{mmol})$ in degassed methanol $(5 \mathrm{~mL})$. After $20 \mathrm{~min} 5$-chloropent-1-yne $(1.1 \mathrm{mmol})$ was added. The resulting mixture was then heated under nitrogen between $40^{\circ} \mathrm{C}$ and $50^{\circ} \mathrm{C}$ for $2 \mathrm{~h}$. The solution was subsequently evaporated under reduced pressure, the residue was dissolved in water, and the solution acidified with hydrochloric acid. The solution was extracted with dichloromethane and then dried over $\mathrm{MgSO}_{4}$. The crude product was purified by column chromatography on silica gel using $n$-hexane / diethyl ether as eluant to give the target compound 13 in almost quantitative yield. Oil; IR (neat) $v_{\max }\left(\mathrm{cm}^{-1}\right) 3500,3291,2936,1718$, 1426; ${ }^{1} \mathrm{H}$ NMR (400 MHz) $\delta 1.84(2 \mathrm{H}, \mathrm{m}) 1.98\left(1 \mathrm{H}, \mathrm{t} J_{t}=2.7\right), 2.34\left(2 \mathrm{H}, \mathrm{td}, J_{t}=7.0, J_{d}=2.7\right), 2.8(2$ $\mathrm{H}, \mathrm{m}), 3.27$ (2 H, s), 8.41 (1 H, bs); ${ }^{13} \mathrm{C}$ NMR $(100 \mathrm{MHz}) \delta 17.3\left(\mathrm{CH}_{2}\right), 27.5,31.5,33.3,69.2(\mathrm{C}), 83.1$, 176.4 (C); MS: $m / z$ (rel. inten.) 158 (24), 113 (45), 99 (100), 65 (95). HRMS calcd for $\mathrm{C}_{7} \mathrm{H}_{10} \mathrm{O}_{2} \mathrm{~S}$ : 158.0402; found: 158.0396.

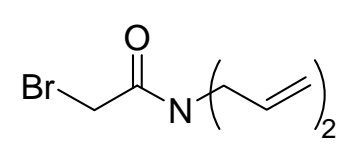

$\mathrm{N}, \mathrm{N}$-Diallyl-2-bromoacetamide (14). ${ }^{3}$ Amide 14 was prepared according to the previously reported procedure. ${ }^{1} \mathrm{H}$ NMR $(300 \mathrm{MHz}) \delta 3.87(2 \mathrm{H}, \mathrm{s}), 4.68(4 \mathrm{H}$, dt, $\left.J_{d}=5.8, J_{t}=1.3\right), 5.29\left(2 \mathrm{H}, \mathrm{dq}, J_{d}=10.4, J_{q}=1.3\right), 5.38\left(2 \mathrm{H}, \mathrm{dq}, J_{d}=17.1\right.$, $\left.J_{q}=1.3\right), 5.93\left(2 \mathrm{H}, \mathrm{ddt}, J_{d l}=17.1, J_{d 2}=10.4, J_{q}=5.8\right)$.

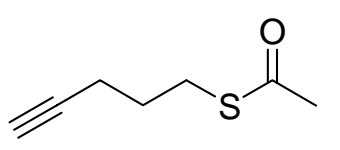

S-Pent-4-ynyl ethanethioate (10). ${ }^{4}$ Thioester 10 was prepared according to the previously reported procedure. ${ }^{1} \mathrm{H}$ NMR $(400 \mathrm{MHz}) \delta 1.81\left(2 \mathrm{H}\right.$, quint, $\left.J_{q}=7.1\right)$, $1.98\left(1 \mathrm{H}, \mathrm{t}, J_{t}=2.6\right), 2.28\left(2 \mathrm{H}, \mathrm{td}, J_{t}=7.0, J_{d}=2.6\right), 2.34(3 \mathrm{H}, \mathrm{s}), 2.98\left(2 \mathrm{H}, \mathrm{t}, J_{t}\right.$ $=7.0)$.

\footnotetext{
${ }^{1}$ Reitz, D. B.; Beak, P.; Farney, R. F.; Helmick, L. S. J. Am. Chem. Soc. 1978, 100, 5428.

${ }^{2}$ Amenta, D. S.; Morton, A. D.; Gilje, J. W.; Edelmann, F. T.; Fischer, A.; Blourock, S. J. Organomet. Chem. 2006, 691, 5065.

${ }^{3}$ Guo, W.; Li, J. J.; Wang, Y. A.; Peng, X. J. Am. Chem. Soc. 2003, 125, 3901.

${ }^{4}$ Benati, L; Calestani, G.; Leardini, R.; Minozzi, M.; Nanni, D.; Spagnolo, P.; Strazzari, S. Org. Lett. 2003,5 , 1313.
} 


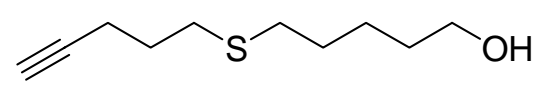

5-(Pent-4-ynylthio)pentan-1-ol (15). S-Pent-4-ynyl ethanethioate $10(1.0 \mathrm{mmol})$ was added at $0{ }^{\circ} \mathrm{C}$ to a solution of potassium hydroxide $(2.2 \mathrm{mmol})$ in degassed methanol $(5 \mathrm{~mL})$. After $10 \mathrm{~min}$, 5-bromopentyl acetate $(1.1 \mathrm{mmol})$ was added. The resulting solution was stirred overnight under nitrogen at room temperature and then additionally stirred for $2 \mathrm{~h}$ at $50{ }^{\circ} \mathrm{C}$. The solution was then evaporated under reduced pressure, the residue was dissolved in water, and the solution acidified with hydrochloric acid. The solution was extracted with dichloromethane and then dried over $\mathrm{MgSO}_{4}$. The crude product was purified by column chromatography on silica gel using $n$-hexane / diethyl ether as eluant to give the target compound $\mathbf{1 5}$ in $80 \%$ yield. Oil; IR $\left(\mathrm{CHCl}_{3}\right) v_{\max }\left(\mathrm{cm}^{-1}\right) 3454,3308,2939,2862 ;{ }^{1} \mathrm{H}$ NMR $(400 \mathrm{MHz}) \delta 1.36(1 \mathrm{H}, \mathrm{bs})$, 1.43-1.51 (2 H, m), 1.55-1.67 (4 H, m), 1.76-1.84 (2 H, m), $1.97\left(1 \mathrm{H}, \mathrm{t}, J_{t}=2.7\right), 2.33\left(2 \mathrm{H}, \mathrm{td}, J_{t}=7.0\right.$, $\left.J_{d}=2.7\right), 2.53\left(2 \mathrm{H}, \mathrm{t}, J_{t}=7.5\right), 2.63\left(2 \mathrm{H}, \mathrm{t}, J_{t}=7.0\right), 3.65\left(2 \mathrm{H}, \mathrm{t}, J_{t}=6.5\right) ;{ }^{13} \mathrm{C} \mathrm{NMR}(100 \mathrm{MHz}) \delta 17.5$ $\left(\mathrm{CH}_{2}\right)$, 25.0 $\left(\mathrm{CH}_{2}\right), 28.3\left(\mathrm{CH}_{2}\right), 29.4\left(\mathrm{CH}_{2}\right), 30.8\left(\mathrm{CH}_{2}\right), 32.0\left(\mathrm{CH}_{2}\right), 32.3\left(\mathrm{CH}_{2}\right), 62.7\left(\mathrm{CH}_{2}\right), 68.4,83.5$ (C); MS: $m / z$ (rel. inten.) $113\left(\mathrm{M}^{+}-73,6\right), 101$ (11), 100 (100), 67 (18). Anal. calcd for $\mathrm{C}_{10} \mathrm{H}_{18} \mathrm{OS}$ : C, 64.46; H, 9.74. Found: C, 64.21; H, 9.70.

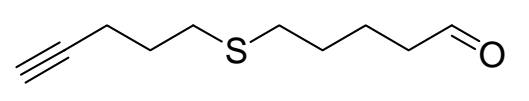

5-(Pent-4-ynylthio)pentanal (16). A dichloromethane (DCM, 2.5 $\mathrm{mL})$ solution of DMSO $(11.0 \mathrm{mmol})$ was added slowly at $-78{ }^{\circ} \mathrm{C}$ to a DCM $(10 \mathrm{~mL})$ solution of oxalyl chloride $(6.5 \mathrm{mmol})$. After stirring for $15 \mathrm{~min}$, a DCM $(5 \mathrm{~mL})$ solution of 5-(pent-4-ynylthio)pentan-1-ol 15 (5 mmol) was added to the above solution. After stirring at $-78{ }^{\circ} \mathrm{C}$ for $1 \mathrm{~h}, 11 \mathrm{mmol}$ of TEA were added at $-78{ }^{\circ} \mathrm{C}$. The reaction mixture was slowly warmed to room temperature, quenched with saturated aqueous ammonium chloride, extracted with DCM, dried over magnesium sulfate, and concentrated. Purification by column chromatography on silica gel ( $n$-hexane / diethyl ether) afforded $4.2 \mathrm{mmol}(82 \%)$ of the desired aldehyde $\mathbf{1 6}$ as a pale yellow oil. IR (neat) $v_{\max }\left(\mathrm{cm}^{-1}\right)$ 3290, 2938, 2862, 2725, 1722, 1432; ${ }^{1} \mathrm{H}$ NMR (400 MHz) $\delta 1.59-1.68$ (2 H, m), $1.70-$ $1.84(4 \mathrm{H}, \mathrm{m}), 1.97\left(1 \mathrm{H}, \mathrm{t}, J_{t}=2.7\right), 2.33\left(2 \mathrm{H}, \mathrm{td}, J_{t}=7.0, J_{d}=2.7\right), 2.47\left(2 \mathrm{H}, \mathrm{td}, J_{t}=7.2, J_{d}=1.7\right)$, $2.54\left(2 \mathrm{H}, \mathrm{t}, J_{t}=7.2\right), 2.63\left(2 \mathrm{H}, \mathrm{t}, J_{t}=7.2\right), 9.78\left(1 \mathrm{H}, \mathrm{t}, J_{t}=1.7\right) ;{ }^{13} \mathrm{C} \mathrm{NMR}(100 \mathrm{MHz}) \delta 17.4\left(\mathrm{CH}_{2}\right)$, $21.1\left(\mathrm{CH}_{2}\right), 28.2\left(\mathrm{CH}_{2}\right), 28.9\left(\mathrm{CH}_{2}\right), 30.8\left(\mathrm{CH}_{2}\right), 31.6\left(\mathrm{CH}_{2}\right), 43.3\left(\mathrm{CH}_{2}\right), 68.9,83.4(\mathrm{C}), 202.1 ; \mathrm{MS}: \mathrm{m} / \mathrm{z}$ (rel. inten.) $155\left(\mathrm{M}^{+}-29,3\right), 127$ (4), 113 (9), 100 (100), 85 (9), 67 (15). Anal. calcd for $\mathrm{C}_{10} \mathrm{H}_{16} \mathrm{OS}$ : C, 65.17; H, 8.75. Found: C, 65.11; H, 8.72.

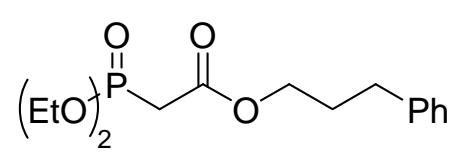

3-Phenyl-propyl (diethylphosphono)acetate (19). Diethylphosphonoacetic acid (2.2 mL of $1 \mathrm{M}$ solution in DCM, $2.2 \mathrm{mmol})$ and DCC (2.2 $\mathrm{mL}$ of a $1 \mathrm{M}$ solution in DCM, $2.2 \mathrm{mmol}$ ) were added to a solution of 3-phenylpropan-1-ol (2 mmol) in DCM (6 mL). The reaction mixture was stirred for $15 \mathrm{~min}$, filtered through a sintered glass, and evaporated. The residue was purified by flash chromatography (50\% to 70\% ethyl acetate / petroleum ether) to give $\mathbf{1 9}$ as a colorless liquid (90\%). IR (neat) $v_{\max }\left(\mathrm{cm}^{-1}\right)$ 2984, 2933, 1736, 1271, 1117, 1052, 1025, 971; ${ }^{1} \mathrm{H}$ NMR $(400 \mathrm{MHz}) \delta 1.35(6 \mathrm{H}$, td, $\left.J_{t}=7.1, J_{d}=0.5\right), 1.95-2.02(2 \mathrm{H}, \mathrm{m}), 2.68-2.74(2 \mathrm{H}, \mathrm{m}), 2.97\left(2 \mathrm{H}, \mathrm{d},{ }^{2} J_{P-H}=21.3\right), 4.14-4.22(6 \mathrm{H}$, $\mathrm{m}), 7.17-7.22(3 \mathrm{H}, \mathrm{m}), 7.26-7.31(2 \mathrm{H}, \mathrm{m}) ;{ }^{13} \mathrm{C} \mathrm{NMR}(100 \mathrm{MHz}) \delta 16.30\left(\mathrm{~d},{ }^{3} J_{P-C}=5.6\right), 30.05\left(\mathrm{CH}_{2}\right)$, $31.93\left(\mathrm{CH}_{2}\right), 34.32\left(\mathrm{CH}_{2}, \mathrm{~d},{ }^{3} J_{P-C}=134\right), 62.62\left(\mathrm{CH}_{2}, \mathrm{~d},{ }^{2} J_{P-C}=6.5\right), 64.80\left(\mathrm{CH}_{2}\right), 126.01,128.37$, 128.42, 140.98 (C), 165.80 (C, d, ${ }^{2} J_{P-C}=5.9$ ); MS: $m / z$ (rel. inten.) 314 (2), 197 (8), 179 (14), 151 (11), 119 (12), 118 (100), 117 (77), 91 (22). Anal. calcd for $\mathrm{C}_{15} \mathrm{H}_{23} \mathrm{O}_{5} \mathrm{P}$ : C, 57.32; H, 7.38. Found: C, 57.22; H, 7.36. 
General procedure for the synthesis of sulfides 1a,b,c,d,f.

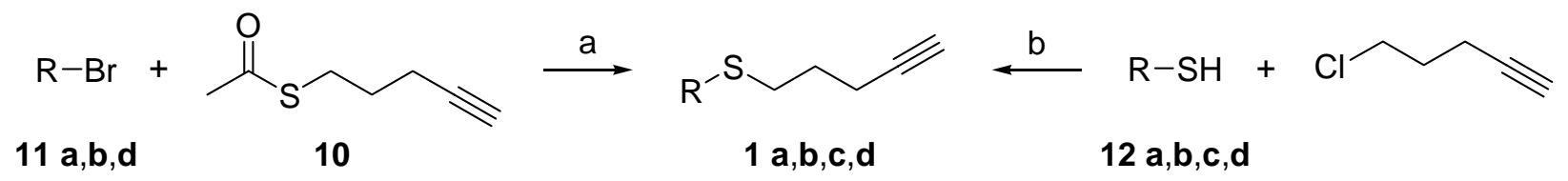

\section{Method A.}

To a degassed solution of $\mathrm{KOH}(2.2 \mathrm{mmol})$ in $\mathrm{MeOH}(5 \mathrm{~mL})$ was added $S$-pent-4-ynyl ethanethioate $10(1.1 \mathrm{mmol})$ and the resulting mixture was stirred at $0{ }^{\circ} \mathrm{C}$ for $15 \mathrm{~min}$ under a nitrogen atmosphere. The proper bromide $\mathbf{1 1}$ was then added and the mixture was stirred for $2 \mathrm{~h}$ at $50^{\circ} \mathrm{C}$. The solution was then evaporated under reduced pressure, the residue was dissolved in water and the solution acidified with hydrochloric acid. The solution was extracted with dichloromethane and then dried over $\mathrm{MgSO}_{4}$. Purification by column chromatography on silica gel ( $n$-hexane / diethyl ether) afforded the desired sulfide $\mathbf{1}$ in good yield.

This method was applied to the preparation of sulfides $\mathbf{1 a}, \mathbf{b}, \mathbf{d}$ starting from the corresponding bromides 11a,b,d.

\section{Method B.}

To a degassed solution of $\mathrm{KOH}(2.2 \mathrm{mmol})$ in $\mathrm{MeOH}(5 \mathrm{~mL})$ was added the proper thiol 12 followed (after $15 \mathrm{~min}$ ) by 5-chloropent-1-yne $(1.1 \mathrm{mmol})$. The resulting mixture was stirred for $2 \mathrm{~h}$ at $50{ }^{\circ} \mathrm{C}$. The solution was then evaporated under reduced pressure, the residue was dissolved in water and the solution acidified with hydrochloric acid. The solution was extracted with dichloromethane and then dried over $\mathrm{MgSO}_{4}$. Purification by column chromatography on silica gel ( $n$-hexane / diethyl ether) afforded the desired sulfide $\mathbf{1}$ in good yield.

This method was applied to the preparation of sulfides 1a-d starting from the corresponding thiols 12a-d.

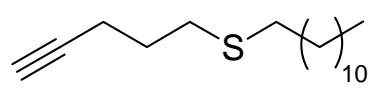

Dodecyl(pent-4-ynyl)sulfane (1a). Colorless liquid (Method A: 95\% yield; Method B: 98\% yield); IR (neat) $v_{\max }\left(\mathrm{cm}^{-1}\right) 3313,2924,2853,1466 ;{ }^{1} \mathrm{H}$ NMR (400 MHz) $\delta 0.88(3 \mathrm{H}, \mathrm{t}, J=7.1), 1.21-1.42(18 \mathrm{H}, \mathrm{m}), 1.54-1.62(2$ H,m), $1.80(2 \mathrm{H}, \mathrm{m}), 1.96(1 \mathrm{H}, \mathrm{t}, J=2.7), 2.32\left(2 \mathrm{H}, \mathrm{td}, J_{t}=7.0, J_{d}=2.6\right), 2.51(2 \mathrm{H}, \mathrm{m}), 2.62(2 \mathrm{H}$, m); ${ }^{13} \mathrm{C}$ NMR (100 MHz) $\delta$ 14.1, $17.5\left(\mathrm{CH}_{2}\right), 22.7\left(\mathrm{CH}_{2}\right), 28.3\left(\mathrm{CH}_{2}\right), 28.9\left(\mathrm{CH}_{2}\right), 29.2\left(\mathrm{CH}_{2}\right), 29.3$ $\left(\mathrm{CH}_{2}\right)$, $29.5\left(\mathrm{CH}_{2}\right), 29,58\left(\mathrm{CH}_{2}\right), 29.61\left(\mathrm{CH}_{2}\right), 29.63\left(\mathrm{CH}_{2}\right), 29.67\left(\mathrm{CH}_{2}\right), 30.8\left(\mathrm{CH}_{2}\right), 31.9\left(\mathrm{CH}_{2}\right), 32.1$ ( $\left.\mathrm{CH}_{2}\right)$, 68.8, 83.6 (C); MS: $\mathrm{m} / z$ (rel. inten.) 155 (M $\mathrm{M}^{+}-113$, 4), 113 (8), 101 (10), 100 (100), 67 (6), 55 (10). Anal. calcd for $\mathrm{C}_{17} \mathrm{H}_{32} \mathrm{~S}$ : C, 76.05; H, 12.01. Found: C, 75.94; H, 12.00.

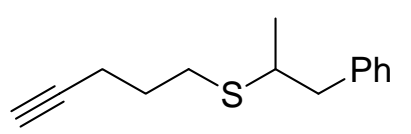

Pent-4-ynyl(1-phenylpropan-2-yl)sulfane (1b). Colorless oil (Method A: 93\% yield; Method B: 98\% yield); IR (neat) $v_{\max }\left(\mathrm{cm}^{-1}\right) 3296,3027,2958$, 2924, 1453; ${ }^{1} \mathrm{H}$ NMR (400 MHz) $\delta 1.23\left(3 \mathrm{H}, \mathrm{d}, J_{d}=6.6\right), 1.75-1.82(2 \mathrm{H}$, m), $1.97\left(1 \mathrm{H}, \mathrm{t}, J_{t}=2.7\right), 2.30\left(2 \mathrm{H}, \mathrm{td}, J_{t}=7.0, J_{d}=2.7\right), 2.64\left(3 \mathrm{H}, \mathrm{t}, J_{t}=7.3\right), 2.96-3.02(2 \mathrm{H}, \mathrm{m})$, 7.16-7.24 (3 H, m), 7.27-7.32 (2 H, m); ${ }^{13} \mathrm{C}$ NMR (100 MHz) $\delta 17.6\left(\mathrm{CH}_{2}\right), 20.7,28.5\left(\mathrm{CH}_{2}\right), 29.4$ $\left(\mathrm{CH}_{2}\right), 41.4\left(\mathrm{CH}_{2}\right), 43.8,68.9,83.6(\mathrm{C}), 126.3,128.3,129.2,139.5$ (C); MS: $m / z$ (rel. inten.) 218 (2), 217 (9), 176 (49), 127 (48), 100 (68), 93 (48), 91 (100), 77 (36), 65 (34). HRMS calcd for $\mathrm{C}_{14} \mathrm{H}_{18} \mathrm{~S}$ : 218.1129; found: 218.1121 . 


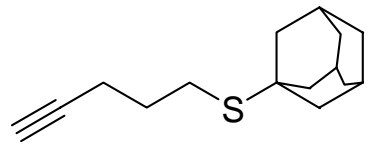

Pent-4-ynyl(1-adamantyl)sulfane (1c). Colorless oil (Method B: 95\% yield); IR (neat) $v_{\max }\left(\mathrm{cm}^{-1}\right) 3308,2904,2849,1449 ;{ }^{1} \mathrm{H}$ NMR $(400 \mathrm{MHz}) \delta 1.65-1.73$ $(6 \mathrm{H}, \mathrm{m}), 1.78(2 \mathrm{H}, \mathrm{m}), 1.87$ (6 H, bd, $\left.J_{d}=2.6\right), 1.97\left(1 \mathrm{H}, \mathrm{t}, J_{t}=2.6\right), 2.04$ (3 $\mathrm{H}, \mathrm{bs}), 2.32\left(2 \mathrm{H}, \mathrm{td}, J_{t}=6.9, J_{d}=2.6\right), 2.63\left(2 \mathrm{H}, \mathrm{t}, J_{t}=7.5\right) ;{ }^{13} \mathrm{C}$ NMR (150 MHz) $\delta 17.8\left(\mathrm{CH}_{2}\right), 24.5\left(\mathrm{CH}_{2}\right), 29.1\left(\mathrm{CH}_{2}\right)$, 29.7, $36.3\left(\mathrm{CH}_{2}\right), 43.6\left(\mathrm{CH}_{2}\right), 44.3(\mathrm{C})$, 68.8, 83.7(C); MS: $m / z$ (rel. inten.) 234 (1), 206 (62), 135 (100). Anal. calcd for $\mathrm{C}_{15} \mathrm{H}_{22} \mathrm{~S}$ : C, 76.86; H, 9.46. Found: C, 76.58; H, 9.44.

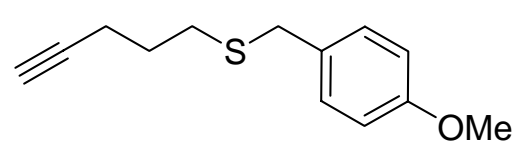

(4-Methoxyphenyl)(pent-4-ynyl)sulfane (1d). Colorless oil (Method A: 95\% yield; Method B: 95\% yield); IR (neat) $v_{\max }\left(\mathrm{cm}^{-1}\right) 3292$, 2934, 2835, 1610, 1510, 1439, 1249; ${ }^{1} \mathrm{H}$ NMR (400 MHz) $\delta 1.76(2$ $\mathrm{H}, \mathrm{m}), 1.94$ (1 H, t, $\left.J_{t}=2.6\right), 2.28\left(2 \mathrm{H}, \mathrm{td}, J_{t}=6.9, J_{d}=2.7\right), 2.51$ (2 $\mathrm{H}, \mathrm{t}, J_{t}=7.1$ ), 3.66 (2 H, s), $3.79(3 \mathrm{H}, \mathrm{s}), 6.84$ (part of AA'BB' system, $2 \mathrm{H}, J=8.8$ ), 7.22 (part of AA'BB' system, $2 \mathrm{H}, J=8.8) ;{ }^{13} \mathrm{C}$ NMR (100 MHz) $\delta 17.5\left(\mathrm{CH}_{2}\right), 28.0\left(\mathrm{CH}_{2}\right), 30.0\left(\mathrm{CH}_{2}\right), 35.5\left(\mathrm{CH}_{2}\right)$, 55.2, 68.8, 83.5 (C), 113.8, 129.8, 130.3 (C), 158.6 (C); MS: m/z (rel. inten.) 220 (21), 173 (15), 121 (100). HRMS calcd for $\mathrm{C}_{13} \mathrm{H}_{16} \mathrm{OS}$ : 220.0922; found: 220.0912.

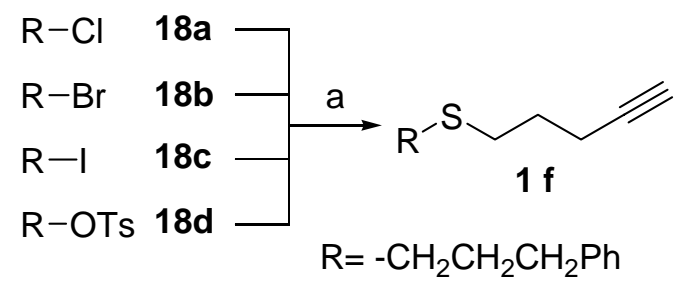

Sulfide 1f was prepared (by Method A) starting from the corresponding chlorinated, brominated, iodinated, and tosilated alcane (18a-d) in order to test the versatility of the procedure. In all cases, the yield of the target sulfide after chromatographic purification (silica gel, $n$-hexane / ethyl ether 95:5 v/v) was almost quantitative.

$\overbrace{\mathrm{Sh}}^{\text {(Pent-4-ynyl)(3-phenylpropyl)sulfane (1f). Colorless oil (Method A: }}$ NMR (400 MHz) $\delta 1.78(2 \mathrm{H}, \mathrm{m}), 1.91(2 \mathrm{H}, \mathrm{m}), 1.96\left(1 \mathrm{H}, \mathrm{t}, J_{t}=2.6\right), 2.32\left(2 \mathrm{H}, \mathrm{td}, J_{t}=7.0, J_{d}=2.6\right)$, $2.53\left(2 \mathrm{H}, \mathrm{dd}, J_{d 1}=7.2, J_{d 2}=7.4\right), 2.62\left(2 \mathrm{H}, \mathrm{dd}, J_{d 1}=7.0, J_{d 2}=7.4\right), 2.72\left(2 \mathrm{H}, \mathrm{dd}, J_{d 1}=7.2, J_{d 2}=7.7\right)$, 7.16-7.21 (3 H, m), 7.26-7.30 (2 H, m); ${ }^{13} \mathrm{C}$ NMR (100 MHz) $\delta 17.5\left(\mathrm{CH}_{2}\right), 28.3\left(\mathrm{CH}_{2}\right), 30.8\left(\mathrm{CH}_{2}\right)$, $31.1\left(\mathrm{CH}_{2}\right), 31.4\left(\mathrm{CH}_{2}\right), 34.8\left(\mathrm{CH}_{2}\right), 68.9$, $83.5(\mathrm{C}), 125.9,128.3,128.4,141.5(\mathrm{C}) ; \mathrm{MS}: \mathrm{m} / z$ (rel. inten.) 218 (3), 176 (6), 118 (43), 117 (84), 100 (100), 91 (37). HRMS calcd for $\mathrm{C}_{14} \mathrm{H}_{18} \mathrm{~S}$ : 218.1129; found: 218.1121.

The preparation of sulfides 1e,g,h was achieved by coupling the starting 2-(pent-4ynylthio)acetic acid 13, via the intermediate acyl chloride, with the corresponding alcohol or amine.

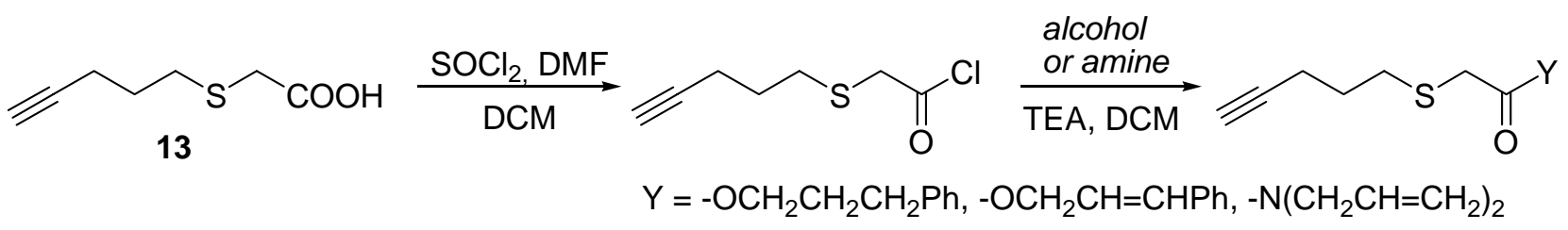




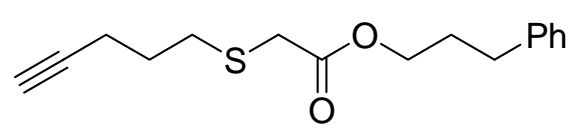

3-Phenylpropyl 2-(pent-4-ynylthio)acetate (1e). An anhydrous DCM (10 mL) solution of 2-(pent-4-ynylthio)acetic acid 13 (2 $\mathrm{mmol})$, freshly distilled $\mathrm{SOCl}_{2}(2.2 \mathrm{mmol})$, and two drops of dry DMF was stirred overnight at room temperature under a nitrogen atmosphere. The intermediate $S$-pent4-ynyl chloromethanethioate was directly added - using a cannula - to a solution of 3-phenylpropan-1ol $(2.2 \mathrm{mmol})$ and TEA $(2.4 \mathrm{mmol})$ in DCM $(10 \mathrm{~mL})$. The resulting solution was stirred for additional $2 \mathrm{~h}$, then water was added and the mixture was extracted twice with DCM. The combined organic phases were dried over $\mathrm{MgSO}_{4}$. Purification by column chromatography on silica gel ( $n$-hexane / diethyl ether) afforded $1.55 \mathrm{mmol}(78 \%)$ of the target sulfide $\mathbf{1 e}$ as a colorless oil. IR (neat) $v_{\max }\left(\mathrm{cm}^{-1}\right)$ 3294, 2939, 1738, 1487; ${ }^{1} \mathrm{H}$ NMR (400 MHz) $\delta 1.84(2 \mathrm{H}, \mathrm{m}), 1.95\left(1 \mathrm{H}, \mathrm{t}, J_{t}=2.7\right), 1.95-2.05(2 \mathrm{H}$, m), $2.33\left(2 \mathrm{H}, \mathrm{td}, J_{t}=6.9, J_{d}=2.7\right), 2.68-2.73(2 \mathrm{H}, \mathrm{m}), 2.75-2.79(2 \mathrm{H}, \mathrm{m}), 3.21(2 \mathrm{H}, \mathrm{s}), 4.16(2 \mathrm{H}, \mathrm{t}$, $\left.J_{t}=6.5\right), 7.16-7.22(3 \mathrm{H}, \mathrm{m}), 7.26-7.31(2 \mathrm{H}, \mathrm{m}) ;{ }^{13} \mathrm{C}$ NMR $(100 \mathrm{MHz}) \delta 17.4\left(\mathrm{CH}_{2}\right), 27.7\left(\mathrm{CH}_{2}\right), 30.1$ $\left(\mathrm{CH}_{2}\right), 31.5\left(\mathrm{CH}_{2}\right), 32.1\left(\mathrm{CH}_{2}\right), 33.6\left(\mathrm{CH}_{2}\right), 64.6\left(\mathrm{CH}_{2}\right), 69.1(\mathrm{C}), 83.2,126.0,128.4,128.5,141.0(\mathrm{C})$, 170.5 (C); MS: $m / z$ (rel. inten.) 276 (2), 158 (3), 119 (13), 118 (100), 99 (9), 91 (84). HRMS calcd for $\mathrm{C}_{16} \mathrm{H}_{20} \mathrm{O}_{2} \mathrm{~S}: 276.1184$; found: 276.1178 .

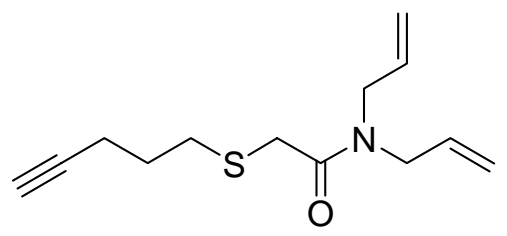

$N, N$-Diallyl-2-(pent-4-ynylthio)acetamide (1g). An anhydrous DCM solution (10 mL) of 2-(pent-4-ynylthio)acetic acid 13 (2 mmol), freshly distilled $\mathrm{SOCl}_{2}(2.2 \mathrm{mmol})$, and two drops of dry DMF was stirred overnight at room temperature under nitrogen. The intermediate S-pent-4-ynyl chloromethanethioate was directly added - using a cannula - to a solution of diallylamine $(2.2 \mathrm{mmol})$ and TEA $(2.4 \mathrm{mmol})$ in DCM $(10 \mathrm{~mL})$. The resulting solution was stirred for additional $2 \mathrm{~h}$, then water was added and the mixture was extracted twice with DCM. The combined organic phases were dried over $\mathrm{MgSO}_{4}$. Purification by column chromatography on silica gel ( $n$-hexane / diethyl ether) afforded $1.7 \mathrm{mmol}(85 \%)$ of the target sulfide $1 \mathrm{~g}$ as a pale yellow oil. IR (neat) $v_{\max }\left(\mathrm{cm}^{-1}\right)$ 3299, 3082, 2931, 1638, 1444, 1442, 927; ${ }^{1} \mathrm{H}$ NMR (400 $\mathrm{MHz}) \delta 1.86(2 \mathrm{H}, \mathrm{m}), 1.96\left(1 \mathrm{H}, \mathrm{t}, J_{t}=2.6\right), 2.32\left(2 \mathrm{H}, \mathrm{td}, J_{t}=6.9, J_{d}=2.6\right), 2.78\left(2 \mathrm{H}, \mathrm{t}, J_{t}=7.1\right), 3.27$ (2 H, s), 3.96-4.01 (4 H, m), 5.13-5.24 (4 H, m), 5.71-5.86 (2 H, m); ${ }^{13} \mathrm{C} \mathrm{NMR} \mathrm{(100} \mathrm{MHz)} \delta 17.3$ $\left(\mathrm{CH}_{2}\right), 27.7\left(\mathrm{CH}_{2}\right), 30.9\left(\mathrm{CH}_{2}\right), 32.9\left(\mathrm{CH}_{2}\right), 47.8\left(\mathrm{CH}_{2}\right), 49.7\left(\mathrm{CH}_{2}\right), 68.9(\mathrm{C}), 83.3,116.7\left(\mathrm{CH}_{2}\right), 117.1$ $\left(\mathrm{CH}_{2}\right), 132.8,132.9,169.2$ (C); MS: $m / z$ (rel. inten.) $139\left(\mathrm{M}^{+}-98,31\right), 138$ (32), 124 (32), 56 (25), 41 (100). Anal. calcd for $\mathrm{C}_{13} \mathrm{H}_{19}$ NOS: C, 65.78; H, 8.07. Found: C, 65.64; H, 8.05. Compound 1g was also prepared in comparable yields by reacting bromoamide 14 with thiolester 10 according to Method A.

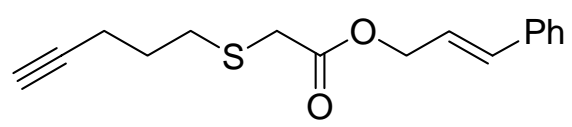

Cinnamyl 2-(pent-4-ynylthio)acetate (1h). An anhydrous DCM $(10 \mathrm{~mL})$ solution of 2-(pent-4-ynylthio)acetic acid 13 (2 mmol), freshly distilled $\mathrm{SOCl}_{2}(2.2 \mathrm{mmol})$, and two drops of dry DMF was stirred overnight at room temperature under nitrogen. The intermediate S-pent-4-ynyl chloromethanethioate was directly added - using a cannula - to a solution of cinnamyl alcohol (2.2 $\mathrm{mmol})$ and TEA $(2.4 \mathrm{mmol})$ in DCM $(10 \mathrm{~mL})$. The resulting solution was stirred for additional $2 \mathrm{~h}$, then water was added and the mixture was extracted twice with DCM. The combined organic phases were dried over $\mathrm{MgSO}_{4}$. Purification by column chromatography on silica gel ( $n$-hexane / diethyl ether) afforded $1.6 \mathrm{mmol}(80 \%)$ of the target sulfide $\mathbf{1 h}$ as a pale yellow oil. IR (neat) $v_{\max }\left(\mathrm{cm}^{-1}\right) 3293,2937$, 1734, 1270, 1125; ${ }^{1} \mathrm{H}$ NMR (400 MHz) $\delta 1.83(2 \mathrm{H}, \mathrm{m}), 1.94\left(1 \mathrm{H}, \mathrm{t}, J_{t}=2.6\right), 2.32\left(2 \mathrm{H}, \mathrm{td}, J_{t}=7.0, J_{d}\right.$ = 2.6), $2.77\left(2 \mathrm{H}, \mathrm{t}, J_{t}=7.4\right), 3.27(2 \mathrm{H}, \mathrm{s}), 4.79\left(2 \mathrm{H}, \mathrm{dd}, J_{d 1}=6.6, J_{d 2}=1.3\right), 6.29\left(1 \mathrm{H}, \mathrm{dt}, J_{d}=15.9, J_{t}\right.$ = 6.6), 6.69 (1 H, bd, $\left.J_{d}=15.9\right), 7.24-7.41(5 \mathrm{H}, \mathrm{m}) ;{ }^{13} \mathrm{C}$ NMR (100 MHz) $\delta 17.4\left(\mathrm{CH}_{2}\right), 27.6\left(\mathrm{CH}_{2}\right)$, $31.5\left(\mathrm{CH}_{2}\right)$, $33.6\left(\mathrm{CH}_{2}\right), 65.9\left(\mathrm{CH}_{2}\right), 69.1(\mathrm{C}), 83.2,122.6,126.6,128.2,128.6,134.7,136.0(\mathrm{C}), 170.2$ 
(C); MS: $m / z$ (rel. inten.) $117\left(\mathrm{M}^{+}-157,100\right), 115$ (30), 91 (12). Anal. calcd for $\mathrm{C}_{16} \mathrm{H}_{18} \mathrm{O}_{2} \mathrm{~S}$ : C, 70.04; H, 6.61. Found: C, 70.00; H, 6.58.

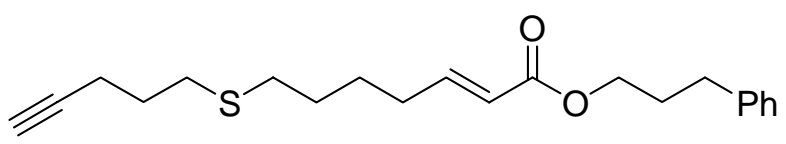

3-Phenylpropyl 7-(pent-4-ynylthio)hept-2-enoate (1i). 3-Phenylpropyl (diethylphosphono)acetate 19 (4.0 mmol) was added at room temperature under nitrogen to a dry THF (12 mL) suspension of sodium hydride $(4.0 \mathrm{mmol})$, followed by addition of a THF (2 mL) solution of 5-(pent-4-ynylthio)pentanal 16 $(4.0 \mathrm{mmol})$. The solution was stirred for $1.5 \mathrm{~h}$. After being quenched into water, the aqueous layer was extracted with chloroform $(3 \times 5 \mathrm{~mL})$. The combined organic phases were dried over $\mathrm{MgSO}_{4}$. Purification by column chromatography on silica gel ( $n$-hexane / diethyl ether) afforded $1.6 \mathrm{mmol}$ (80\%) of the target sulfide $\mathbf{1 i}$ as a pale yellow oil. IR $\left(\mathrm{CDCl}_{3}\right) v_{\max }\left(\mathrm{cm}^{-1}\right) 3293,2935,2858,1718$, 1654, 1454, 1266; ${ }^{1} \mathrm{H}$ NMR (400 MHz) $\delta 1.52-1.68$ (4 H, m), 1.80 (2 H, quint, $J_{\text {quint }}=7.0$ ), 1.95-2.03 (3 H, m), $2.23\left(2 \mathrm{H}, \mathrm{qd}, J_{q}=6.8, J_{d}=1.5\right), 2.32$ ( $\left.2 \mathrm{H}, \mathrm{td}, J_{t}=6.9, J_{d}=2.5\right), 2.53\left(2 \mathrm{H}, \mathrm{t}, J_{t}=6.9\right), 2.62$ (2 H, dd, $\left.J_{d 1}=7.4, J_{d 2}=6.9\right), 2.71\left(2 \mathrm{H}\right.$, dd, $\left.J_{d 1}=7.4, J_{d 2}=8.1\right), 4.15\left(2 \mathrm{H}, \mathrm{t}, J_{t}=6.5\right), 5.84\left(1 \mathrm{H}, \mathrm{dt}, J_{d}=\right.$ 15.7, $\left.J_{t}=1.7\right), 6.94\left(1 \mathrm{H}, \mathrm{dt}, J_{d}=15.7, J_{t}=6.9\right), 7.16-7.22(3 \mathrm{H}, \mathrm{m}), 7.26-7.31(2 \mathrm{H}, \mathrm{m}) ;{ }^{13} \mathrm{C}$ NMR $(100$ MHz) $\delta 17.5\left(\mathrm{CH}_{2}\right), 28.1\left(\mathrm{CH}_{2}\right), 28.3\left(\mathrm{CH}_{2}\right), 29.0\left(\mathrm{CH}_{2}\right), 30.2\left(\mathrm{CH}_{2}\right), 30.8\left(\mathrm{CH}_{2}\right), 31.7\left(\mathrm{CH}_{2}\right), 31.7$ $\left(\mathrm{CH}_{2}\right), 32.2\left(\mathrm{CH}_{2}\right), 63.5\left(\mathrm{CH}_{2}\right), 68.9(\mathrm{C}), 83.5,121.5,125.9,128.4,128.4,141.2(\mathrm{C}), 148.8,166.6(\mathrm{C})$; MS: $m / z$ (rel. inten.) 344 (2), 181 (6), 153 (5), 141 (5), 118 (29), 117 (39), 100 (100), 91 (62). HRMS calcd for $\mathrm{C}_{21} \mathrm{H}_{28} \mathrm{O}_{2} \mathrm{~S}$ : 344.1810; found: 344.1803.

\section{General Procedure for the Reactions of Sulfides 1a-f.}

A degassed toluene solution $(16.0 \mathrm{~mL})$ of thiophenol $(1.1 \mathrm{mmol})$ and ACCN $(0.15 \mathrm{mmol})$ was added by siringe pump over 2 hours under a nitrogen atmosphere to a boiling, degassed toluene solution (10.0 $\mathrm{mL})$ containing the appropriate sulfide 1a-f $(1.0 \mathrm{mmol})$. The resulting mixture was refluxed for additional $1 \mathrm{~h}$, until virtual disappearance of the starting materials. The solution was then evaporated under reduced pressure and the crude product was purified by column chromatography on silica gel, using $n$-hexane / diethyl ether as eluant, to give the desidered reduced product $\mathbf{4 a - f}$. Reaction products 4b and 4f are identical: for spectral data of $\mathbf{4 f}$, see data of $\mathbf{4 b}$; copies of NMR spectral data of 1-propylbenzene obtained from both $\mathbf{1 b}$ and $\mathbf{1 f}$ are reported separately below.

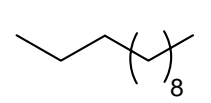

Dodecane (4a). Colorless liquid; ${ }^{1} \mathrm{H}$ NMR (600 MHz) $\delta 0.87$ (6 H, t, $\left.J_{t}=7.0\right), 1.21-1.27$ $(20 \mathrm{H}, \mathrm{m}) ;{ }^{13} \mathrm{C}$ NMR $(150 \mathrm{MHz}) \delta 14.1,22.7\left(\mathrm{CH}_{2}\right), 29.4\left(\mathrm{CH}_{2}\right), 29.6\left(\mathrm{CH}_{2}\right), 29.7$ $\left(\mathrm{CH}_{2}\right), 31.9\left(\mathrm{CH}_{2}\right)$; MS: $m / z$ (rel. inten.) 170 (6), 127 (4), 113 (4), 85 (32), 71 (67), 57 (100), 43 (51).

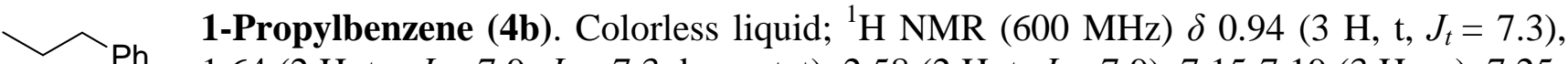
1.64 ( $2 \mathrm{H}, \mathrm{tq}, J_{t}=7.9, J_{q}=7.3$, br sextet), 2.58 ( $\left.2 \mathrm{H}, \mathrm{t}, J_{t}=7.9\right), 7.15-7.19(3 \mathrm{H}, \mathrm{m}), 7.25-$ 7.29 (2 H, m); ${ }^{13} \mathrm{C}$ NMR (150 MHz) $\delta$ 13.8, $24.6\left(\mathrm{CH}_{2}\right), 38.1\left(\mathrm{CH}_{2}\right), 125.6,128.2,128.4,142.7(\mathrm{C})$.

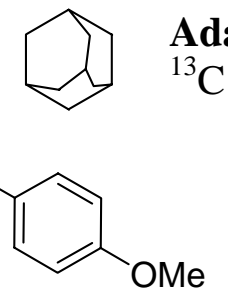

Adamantane (4c). White solid; ${ }^{1} \mathrm{H}$ NMR (400 MHz) $\delta$ 1.73-1.77 (12 H, m), 1.87 (4 H, bs); ${ }^{13} \mathrm{C}$ NMR (100 MHz) $\delta$ 28.3, $37.8\left(\mathrm{CH}_{2}\right)$.

1-Methoxy-4-methylbenzene (4d). Liquid; ${ }^{1} \mathrm{H}$ NMR (400 MHz) $\delta 2.28$ (3 H, s), 3.77 (3 H, s), 6.80 (2 H, part of AA'BB', $J=8.7)$, 7.08 (2 H, part of AA'BB', $J=8.7) ;{ }^{13} \mathrm{C}$ NMR (100 MHz) $\delta$ 20.4, 55.2, 113.7, 129.8 (C), 129.9, 157.4 (C). 


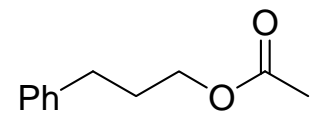

3-Phenylpropyl acetate (4e). ${ }^{5}$ Oil; ${ }^{1} \mathrm{H}$ NMR (400 MHz) $\delta$ 1.92-2.00 (2 H, m), 2.05 (3 H, s), 2.69 (2 H, t, $\left.J_{t}=7.4\right), 4.09$ (2 H, t, $\left.J_{t}=6.7\right)$, 7.17-7.22 (3 H, m), 7.26-7.31 (2 H, m); ${ }^{13} \mathrm{C}$ NMR (100 MHz) $\delta$ 20.9, $30.2\left(\mathrm{CH}_{2}\right), 32.2\left(\mathrm{CH}_{2}\right), 63.8$ $\left(\mathrm{CH}_{2}\right), 126.0,128.3,128.4,141.2$ (C), 171.1 (C); MS: m/z (rel. inten.) 178 (1), 118 (75), 117 (100), 91 (38), 43 (37).

\section{General Procedure for the Reactions of Sulfides 1g-i.}

A degassed toluene solution $(16.0 \mathrm{~mL})$ of thiophenol $(1.1 \mathrm{mmol})$ and ACCN $(0.15 \mathrm{mmol})$ was added by siringe pump over 4 hours under a nitrogen atmosphere to a boiling, degassed toluene solution (10.0 $\mathrm{mL})$ containing the appropriate sulfide $1 \mathrm{~g}-\mathbf{i}(1.0 \mathrm{mmol})$. The resulting mixture was refluxed for additional $1 \mathrm{~h}$, until virtual disappearance of the starting materials. The solution was then evaporated under reduced pressure and the crude product was purified by column chromatography on silica gel, using $n$-hexane / diethyl ether as eluant, to give the desidered cyclized product 6-8.

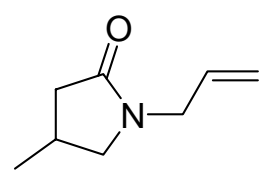

1-Allyl-4-methylpyrrolidin-2-one (6). ${ }^{6}$ Elution with $n$-hexane / diethyl ether 70/30 v/v gave $0.75 \mathrm{mmol}$ of the target compound as a pale yellow oil (75\%); IR (neat) $v_{\max }\left(\mathrm{cm}^{-1}\right)$ 2961, 2872, 1690, 1440, 1419; ${ }^{1} \mathrm{H}$ NMR $(400 \mathrm{MHz}) \delta 1.13\left(3 \mathrm{H}, \mathrm{d}, J_{d}=\right.$ 6.8), $2.05\left(1 \mathrm{H}\right.$, dd, $\left.J_{d 1}=16.5, J_{d 2}=6.8\right), 2.39-2.49(1 \mathrm{H}, \mathrm{m}), 2.57\left(1 \mathrm{H}, \mathrm{dd}, J_{d l}=\right.$ 16.5, $\left.J_{d 2}=8.5\right), 2.92\left(1 \mathrm{H}, \mathrm{dd}, J_{d 1}=9.7, J_{d 2}=6.0\right), 3.46\left(1 \mathrm{H}, \mathrm{bt}, J_{t}=7.8\right), 3.88(2 \mathrm{H}$, $\left.\mathrm{d}, J_{d}=6.0\right), 5.15-5.21(2 \mathrm{H}, \mathrm{m}), 5.67-5.78(1 \mathrm{H}, \mathrm{m}) ;{ }^{13} \mathrm{C}$ NMR $(100 \mathrm{MHz}) \delta 19.8,26.2,39.3\left(\mathrm{CH}_{2}\right), 45.0$ $\left(\mathrm{CH}_{2}\right), 54.0\left(\mathrm{CH}_{2}\right), 117.7\left(\mathrm{CH}_{2}\right), 132.4,174.2$ (C); MS: m/z (rel. inten.) 139 (45), 124 (13), 112 (7), 96 (12), 70 (100), 41 (41).

4-Benzyl-dihydrofuran-2(3H)-one (7). ${ }^{7}$ Elution with $n$-hexane / diethyl ether 80/20 $\mathrm{v} / \mathrm{v}$ gave $0.75 \mathrm{mmol}$ of the target compound as a pale yellow oil (75\%); IR (neat) $v_{\max }$ $\left(\mathrm{cm}^{-1}\right)$ 2971, 2923, 1777; ${ }^{1} \mathrm{H}$ NMR $(400 \mathrm{MHz}) \delta 2.29\left(1 \mathrm{H}, \mathrm{dd}, J_{d l}=17.6, J_{d 2}=6.9\right)$, 2.60 ( $\left.1 \mathrm{H}, \mathrm{dd}, J_{d 1}=17.5, J_{d 2}=8.1\right), 2.76\left(1 \mathrm{H}, \mathrm{d}, J_{d}=3.8\right), 2.78\left(1 \mathrm{H}, \mathrm{d}, J_{d}=2.1\right), 2.80-$ $2.91(1 \mathrm{H}, \mathrm{m}), 4.03\left(1 \mathrm{H}, \mathrm{dd}, J_{d 1}=9.2, J_{d 2}=6.0\right)$, $4.33\left(1 \mathrm{H}, \mathrm{dd}, J_{d 1}=9.2, J_{d 2}=6.9\right)$, 7.13-7.17 (2 H, m), 7.22-7.27 (1 H, m), 7.29-7.35 (2 H, m); ${ }^{13} \mathrm{C}$ NMR (100 MHz) $\delta 34.2\left(\mathrm{CH}_{2}\right), 37.1$, $38.9\left(\mathrm{CH}_{2}\right), 72.6\left(\mathrm{CH}_{2}\right), 126.8,128.6,128.8,138.2$ (C), 176.8 (C); MS: $m / z$ (rel. inten.) 176 (24), 117 (12), 92 (59), 91 (100), 65 (13).

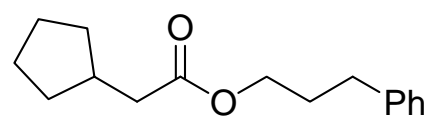

3-Phenylpropyl 2-cyclopentylacetate (8). Purification was performed on basic alumina using as eluant a solution of $n$-hexane / diethyl ether $80 / 20$ $\mathrm{v} / \mathrm{v}$. The chromatography column gave $0.70 \mathrm{mmol}$ of the target compound as a colorless oil (70\%); IR $\left(\mathrm{CDCl}_{3}\right) v_{\max }\left(\mathrm{cm}^{-1}\right)$ 2959, 1726; ${ }^{1} \mathrm{H}$ NMR (400 MHz) $\delta$ 1.38-1.78 (8 H, m), 1.95-2.04 (2 H, m), 2.18-2.29 (1 H, m), 2.66-2.74 (3 H, m), 4.10-4.27 (3 H, m), 7.16-7.23 (3 H, m), 7.27-7.32 (2 H, m); ${ }^{13} \mathrm{C}$ NMR (100 MHz) $\delta 25.6\left(\mathrm{CH}_{2}\right), 26.5\left(\mathrm{CH}_{2}\right), 28.7$ $\left(\mathrm{CH}_{2}\right), 30.2\left(\mathrm{CH}_{2}\right), 32.1\left(\mathrm{CH}_{2}\right), 43.5,64.8\left(\mathrm{CH}_{2}\right), 126.1,128.4,128.5,140.9(\mathrm{C}), 175.2(\mathrm{C})$; MS: $\mathrm{m} / z$ (rel. inten.) 118 ( $\left.\mathrm{M}^{+}-128,100\right), 117$ (44), 91 (32), 81 (34), 69 (7). Compound 8 partially decomposes upon chromatography on both silica gel and (to a minor extent) basic alumina: chromatographic separation must be therefore as fast as possible. Due to decomposition problems during purification and absence of molecular ion in the mass spectrum, for compound $\mathbf{8}$ we could obtain neither good

\footnotetext{
${ }^{5}$ Chandrasekhar, S.; Ramachandar, T.; Reddy, M. V.; Takhi, M. J. Chem. Soc. 2000, 65, 4729.

${ }^{6}$ Ozaki, S.; Matsushita, H.; Ohmori, H. J. Chem. Soc., Perkin Trans. 1, 1993, 2339.

${ }^{7}$ Van Heerden, P. S.; Bezuidenhoudt, B. C. B; Ferriera, D. Tetrahedron 1996, 52, 12313.
} 
elemental analysis nor accurate mass determination. The structure is however clearly confirmed by the ${ }^{1} \mathrm{H}$ and ${ }^{13} \mathrm{C}$ NMR spectra reported above.<smiles>CCCCOCCCC(=O)OCCCc1ccccc1</smiles>

3-Phenylpropyl 4-butoxybutanoate (9). A degassed toluene solution $(13.0 \mathrm{~mL})$ of thiophenol $(1.1 \mathrm{mmol})$ and ACCN $(0.15$ mmol) was added by siringe pump over 2 hours under a nitrogen atmosphere to a boiling degassed toluene solution $(13.0 \mathrm{~mL})$ containing 3-phenylpropyl 2-(pent-4-ynylthio)acetate 1e $(1.0 \mathrm{mmol})$ and 1-(vinyloxy)butane $(5.0 \mathrm{mmol})$. The resulting mixture was refluxed for additional $1 \mathrm{~h}$, until virtual disappearance of the starting materials. The solution was then evaporated under reduced pressure and the crude product was purified by column chromatography on silica gel using increasing $n$-hexane / diethyl ether gradients to give 0.4 mmol of 9 as a yellow oil (40\%). IR (neat) $v_{\max }\left(\mathrm{cm}^{-1}\right)$ 2978, 2931, 1742; ${ }^{1} \mathrm{H}$ NMR (400 MHz) $\delta 0.91$ (3 H, t, $\left.J_{t}=7.3\right), 1.31-1.43$ (2 H, m), 1.50-1.58 (2 H, m), 1.86-2.00 (4 H, m), 2.40 (2 H, t, $\left.J_{t}=7.4\right), 2.69\left(2 \mathrm{H}, \mathrm{t}, J_{t}=7.7\right), 3.40(2 \mathrm{H}$, $\left.\mathrm{t}, J_{t}=6.7\right), 3.40\left(2 \mathrm{H}, \mathrm{t}, J_{t}=6.2\right), 4.09\left(2 \mathrm{H}, \mathrm{t}, J_{t}=6.7\right), 7.16-7.22(3 \mathrm{H}, \mathrm{m}), 7.25-7.31(2 \mathrm{H}, \mathrm{m}) ;{ }^{13} \mathrm{C}$ NMR (100 MHz) $\delta$ 13.9, $19.3\left(\mathrm{CH}_{2}\right), 25.1\left(\mathrm{CH}_{2}\right), 30.2\left(\mathrm{CH}_{2}\right), 31.1\left(\mathrm{CH}_{2}\right), 31.8\left(\mathrm{CH}_{2}\right), 32.1\left(\mathrm{CH}_{2}\right), 63.6$ $\left(\mathrm{CH}_{2}\right)$, $69.6\left(\mathrm{CH}_{2}\right), 70.7\left(\mathrm{CH}_{2}\right), 126.0,128.4,128.4,141.2(\mathrm{C}), 173.6(\mathrm{C}) ; \mathrm{MS}: \mathrm{m} / z$ (rel. inten.) 278 (1), 119 (18), 118 (100), 117 (59), 91 (31), 57 (8). Anal. calcd for $\mathrm{C}_{17} \mathrm{H}_{26} \mathrm{O}_{3}$ : C, 73.34; H, 9.41. Found: C, 73.19; H, 9.38. 


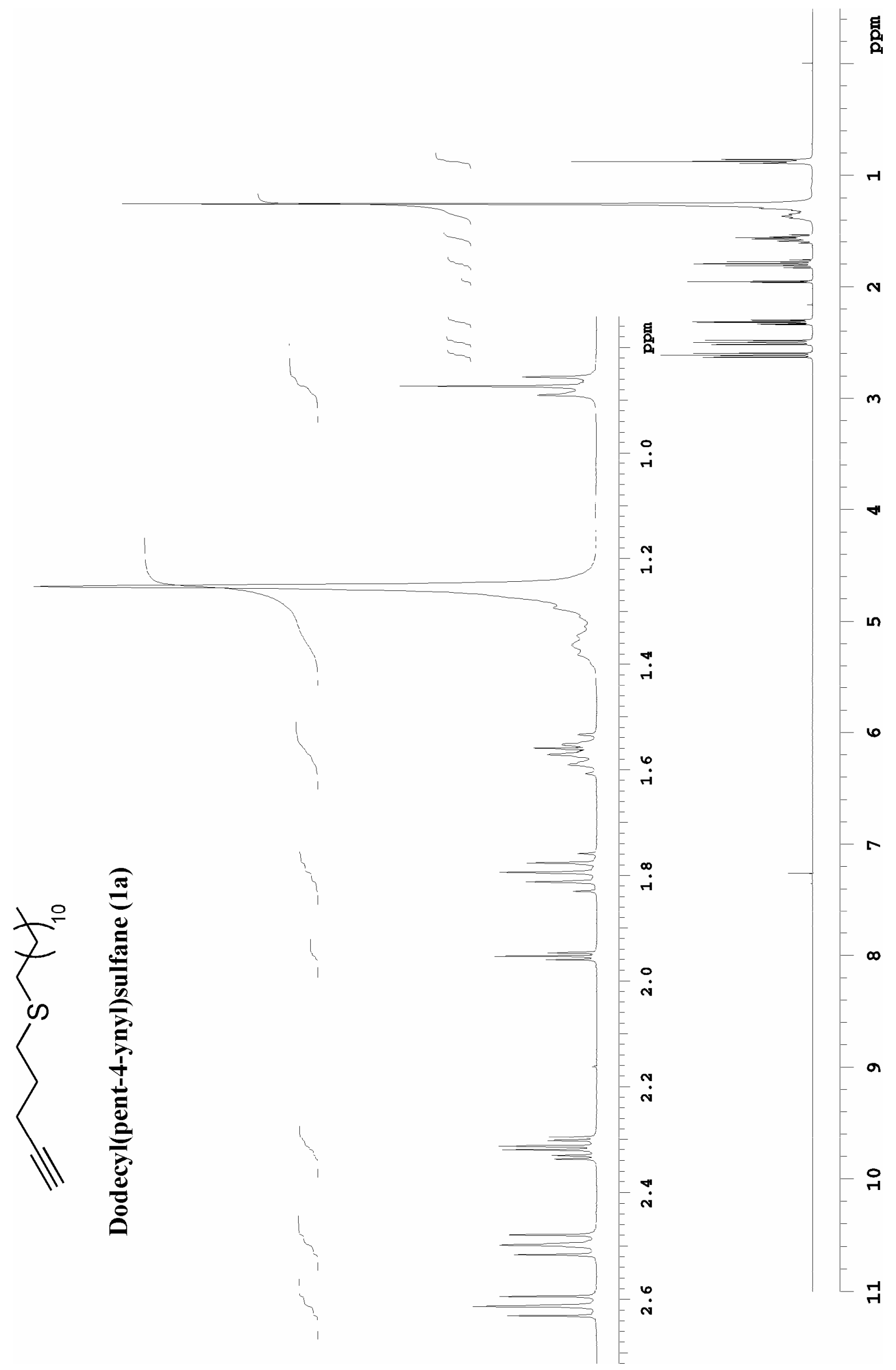




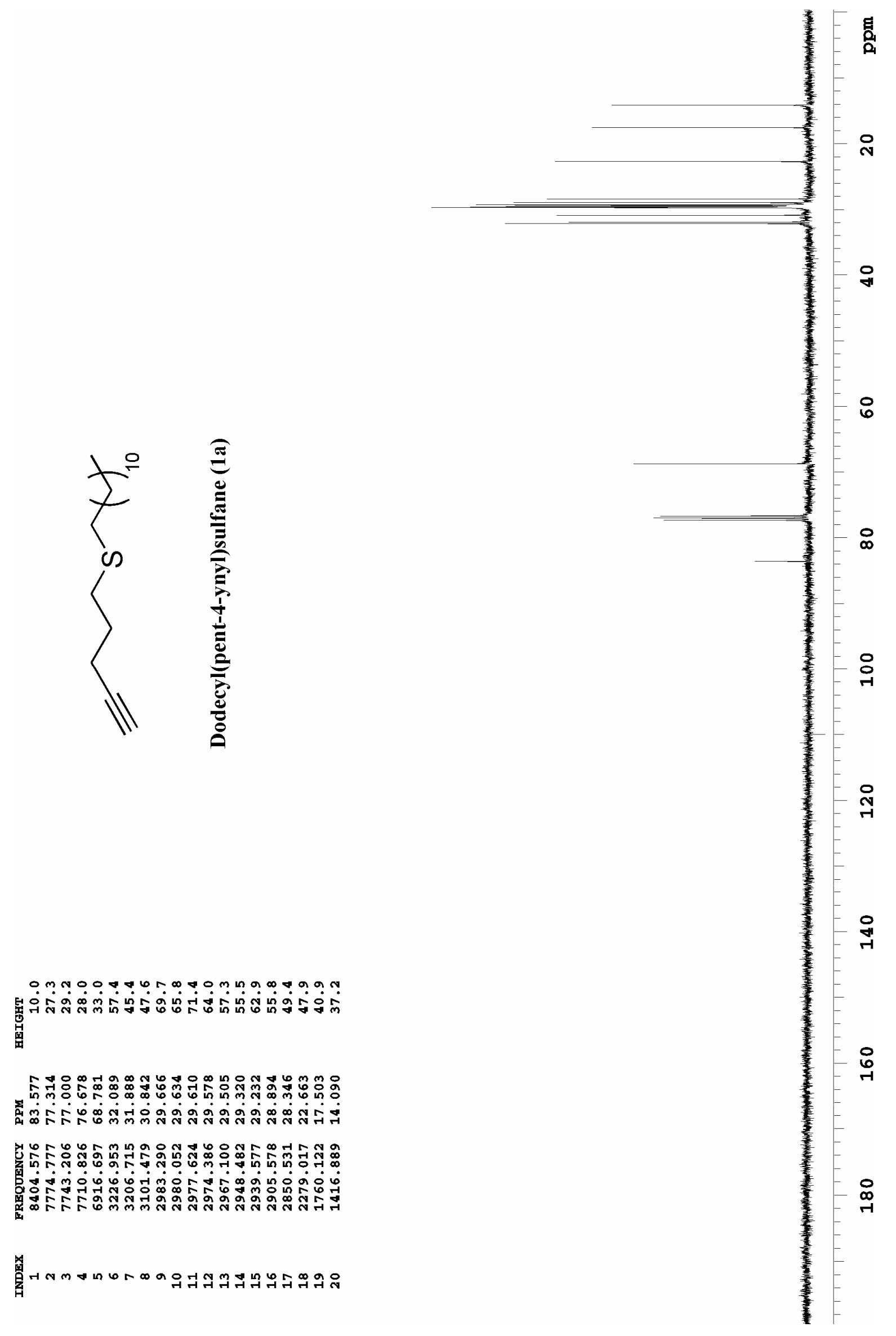




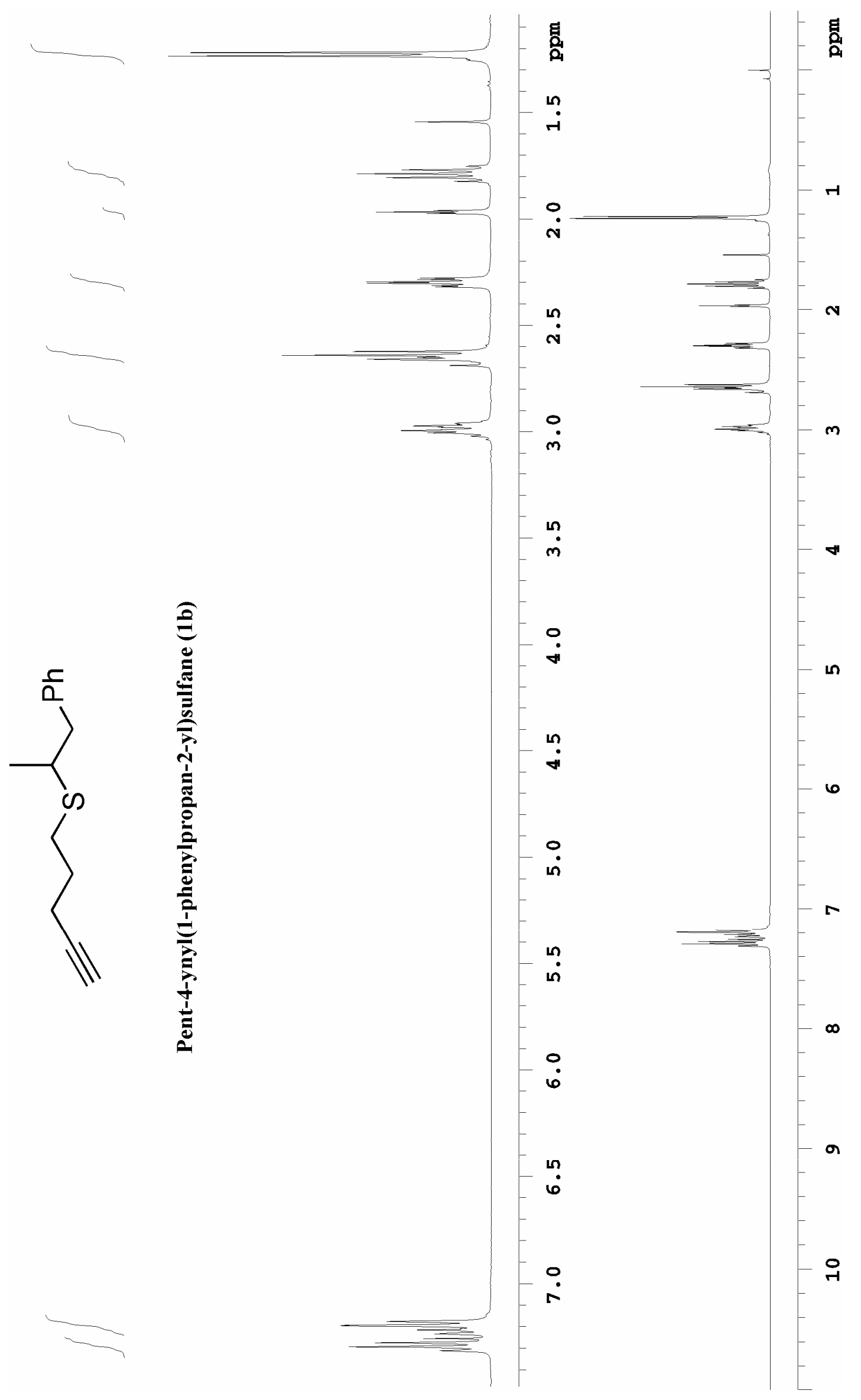




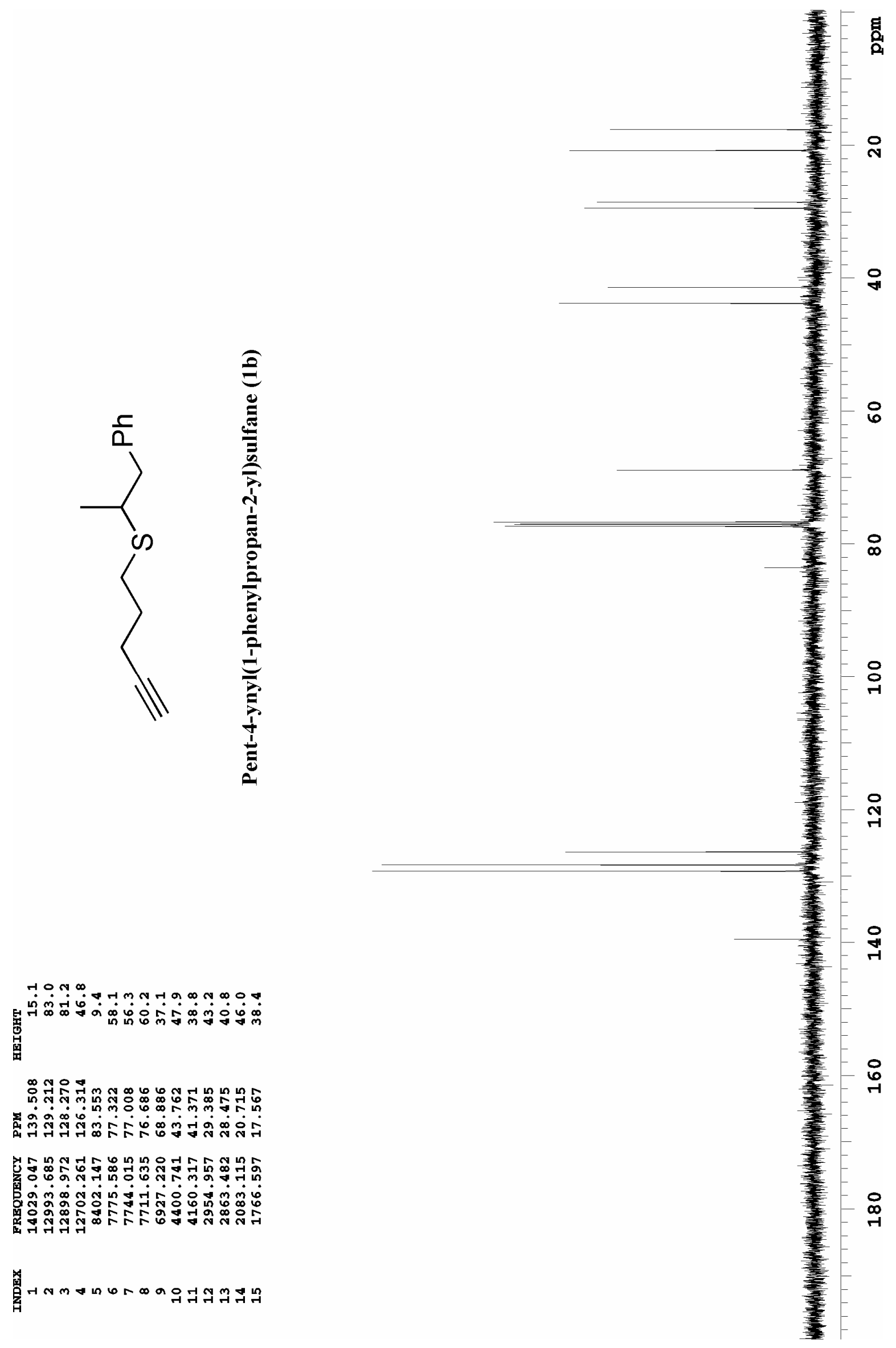




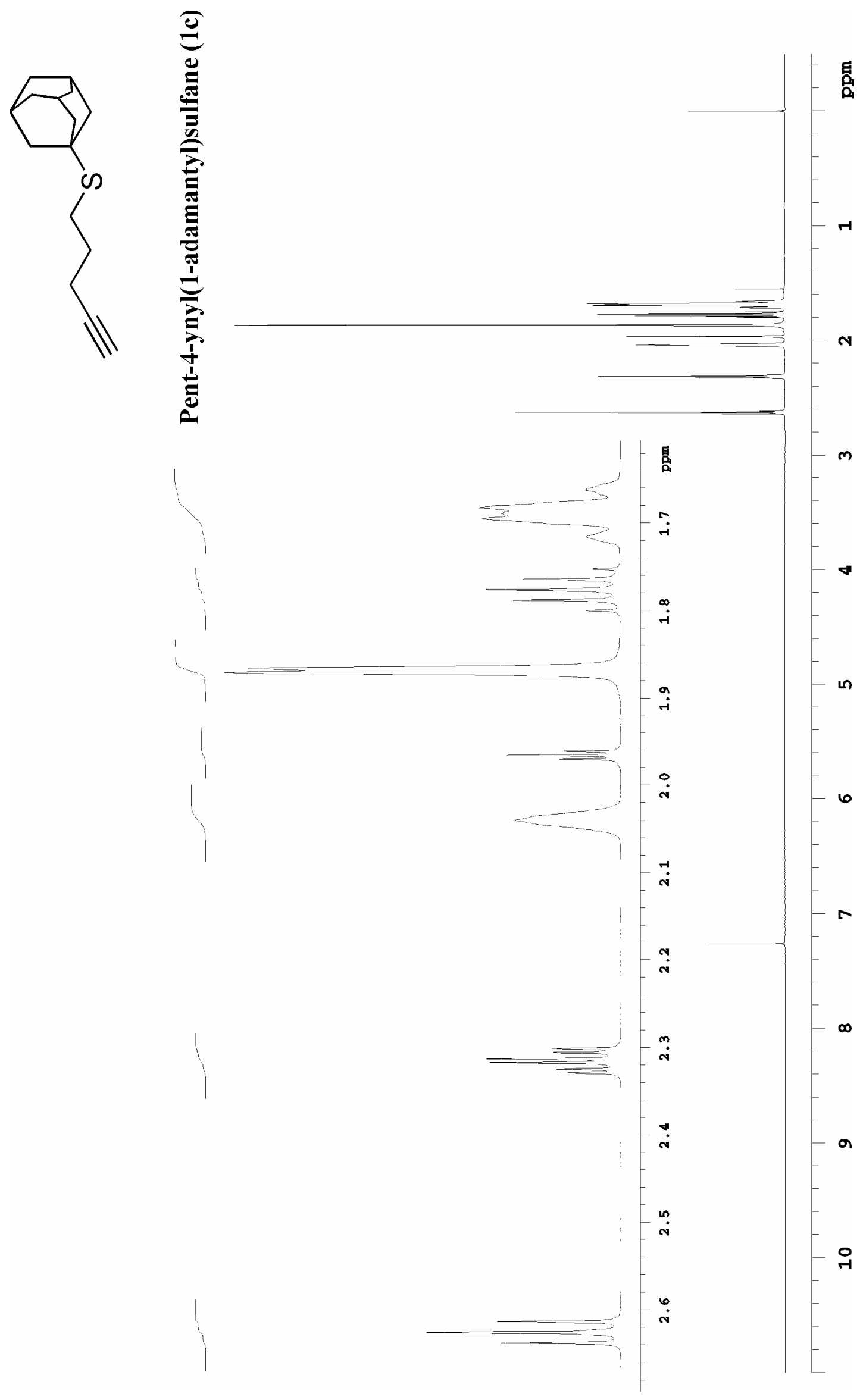




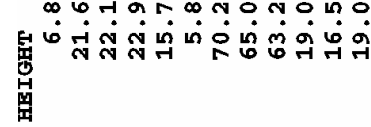

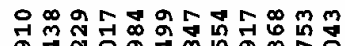

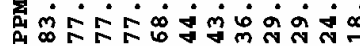

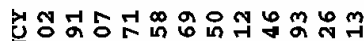

일

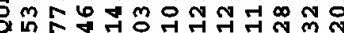

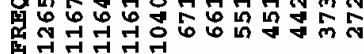

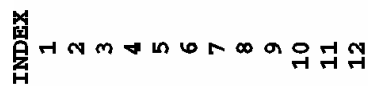

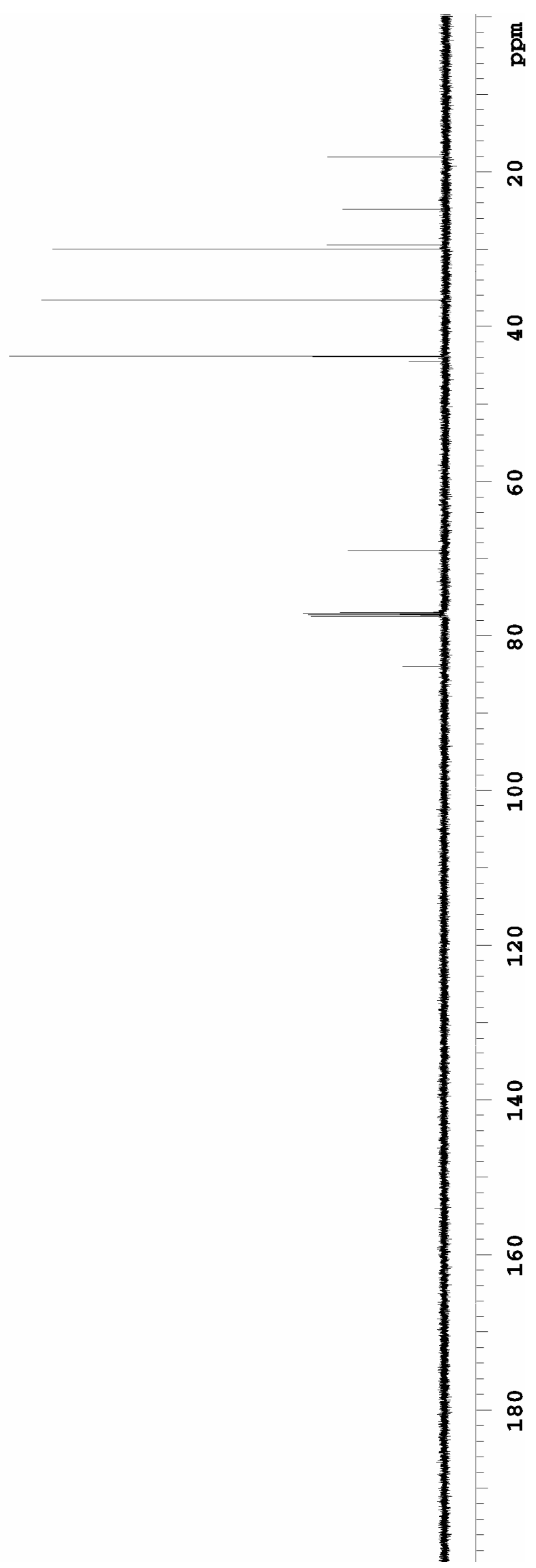

8

$\stackrel{8}{\circ}$

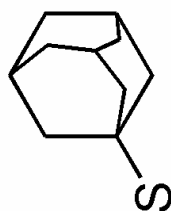

$<$

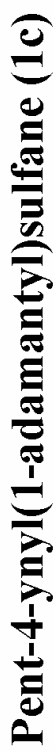

ฉ

疋

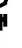

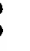

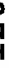

욤 


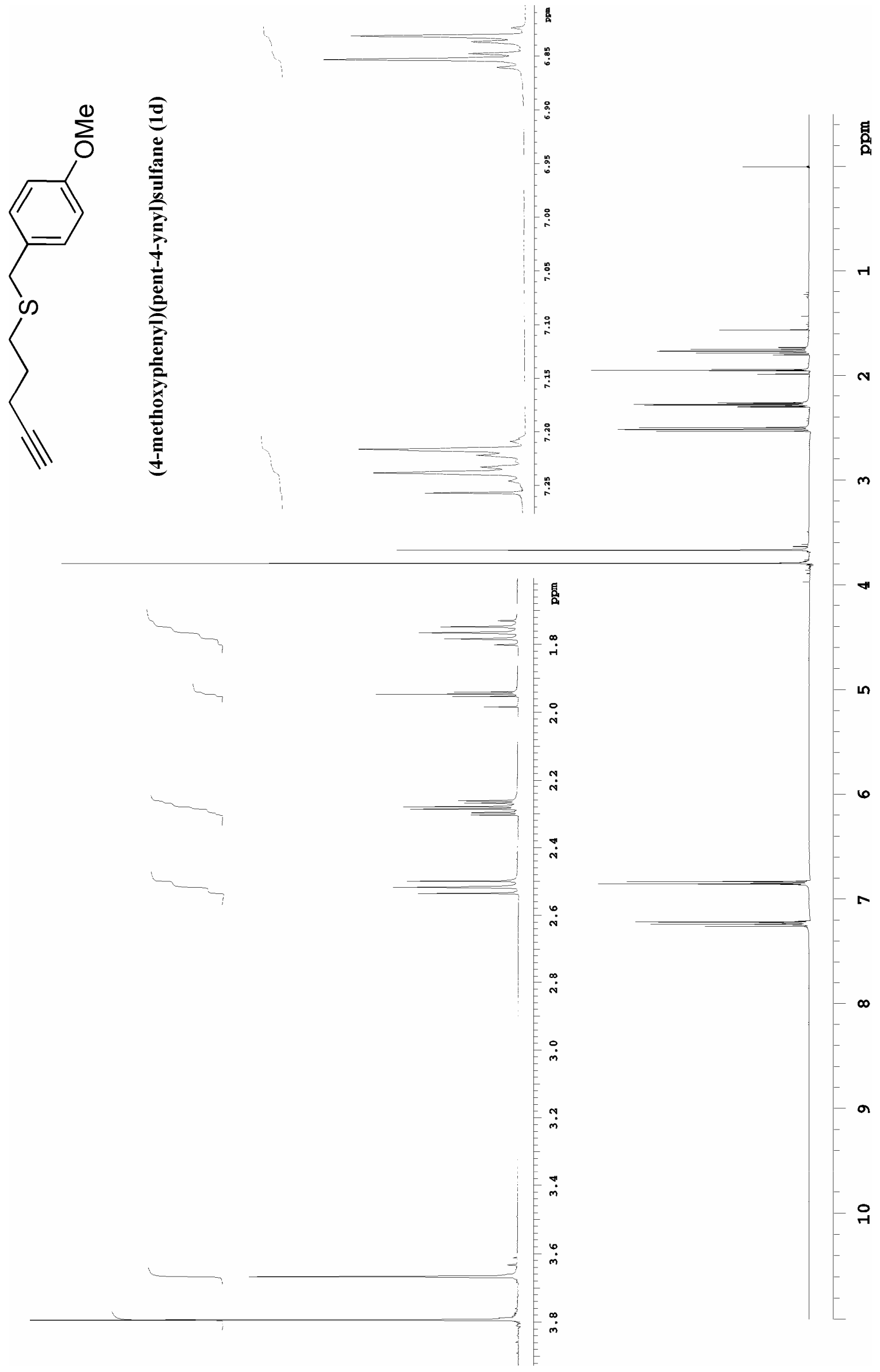




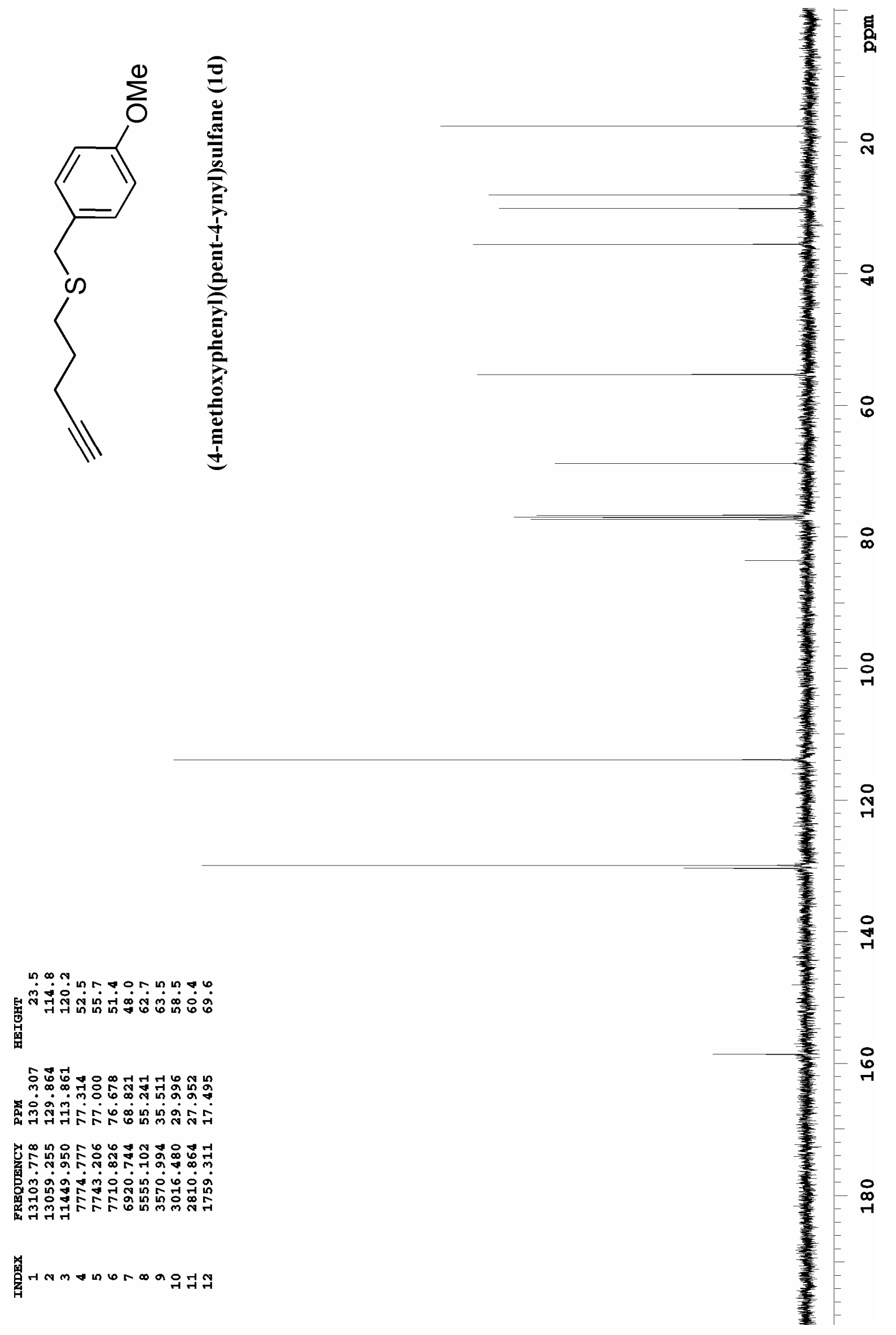




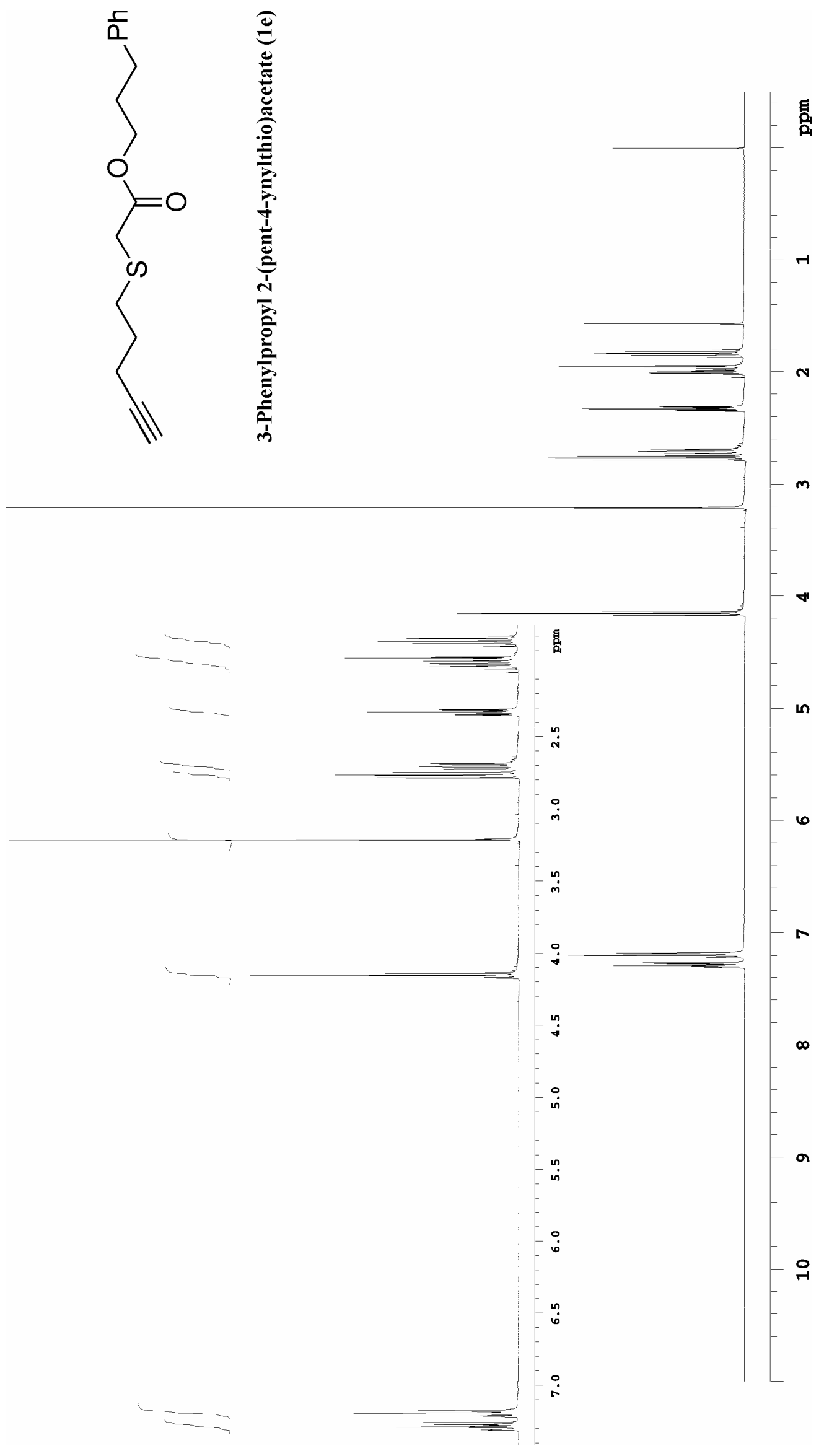




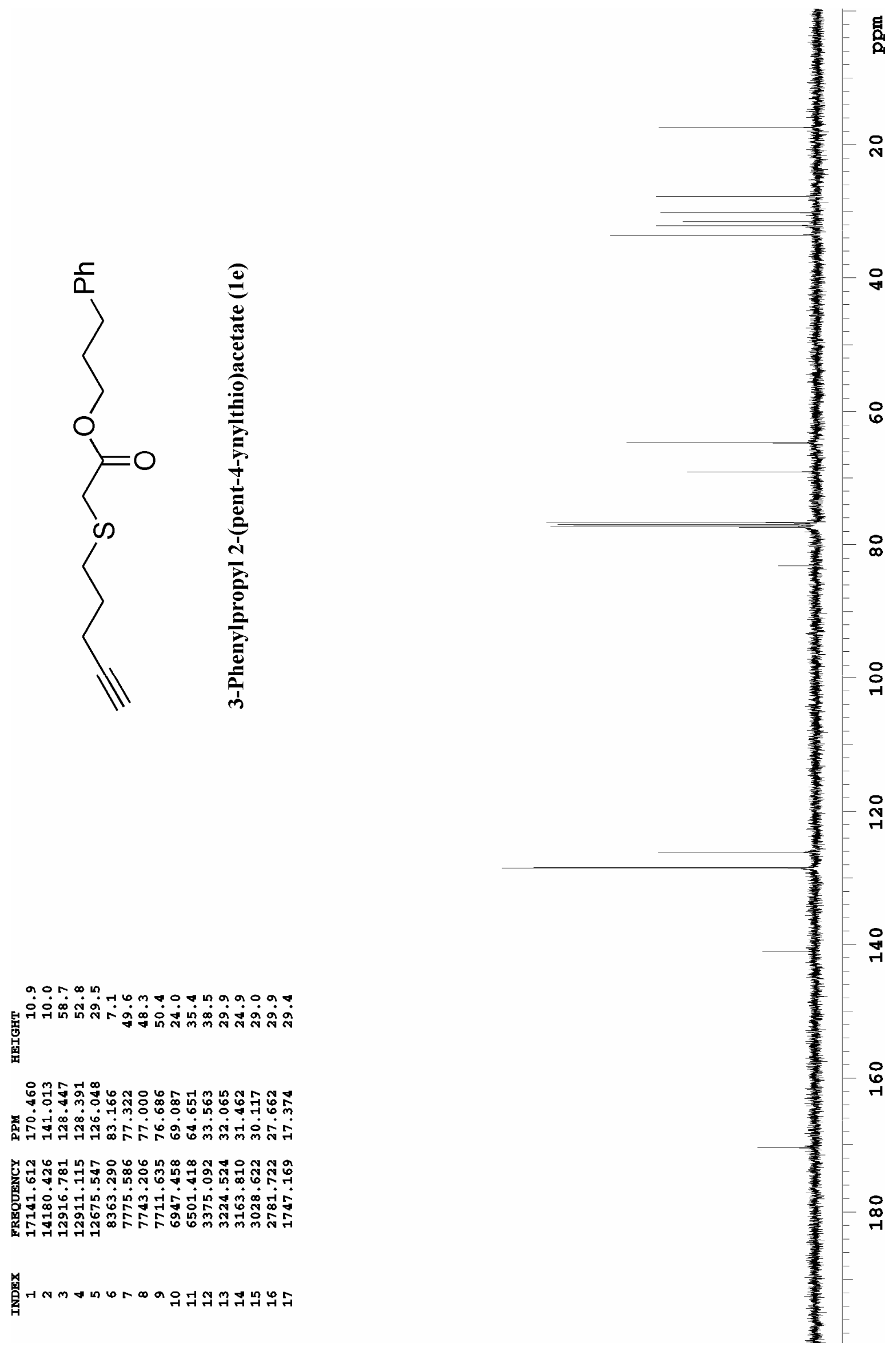




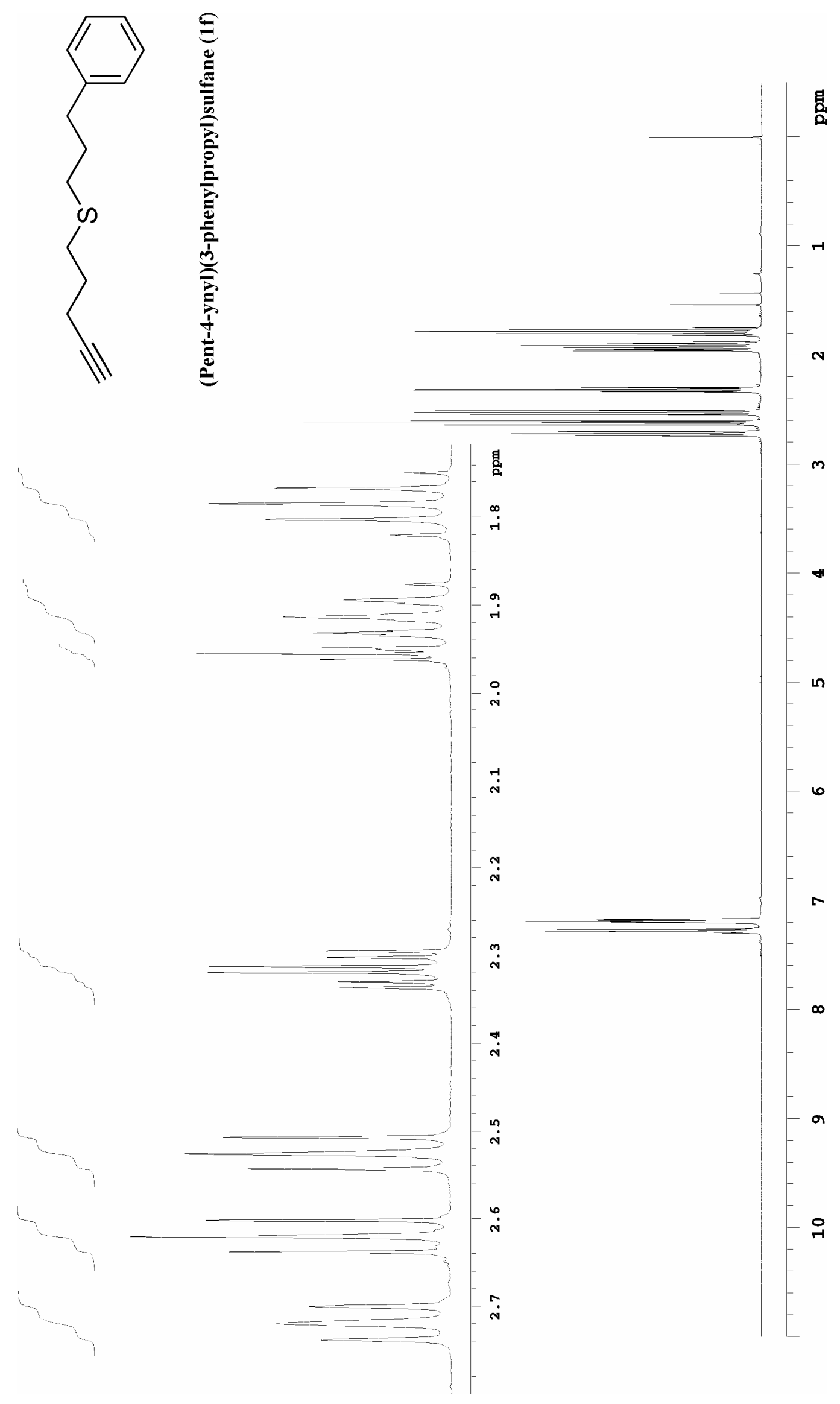




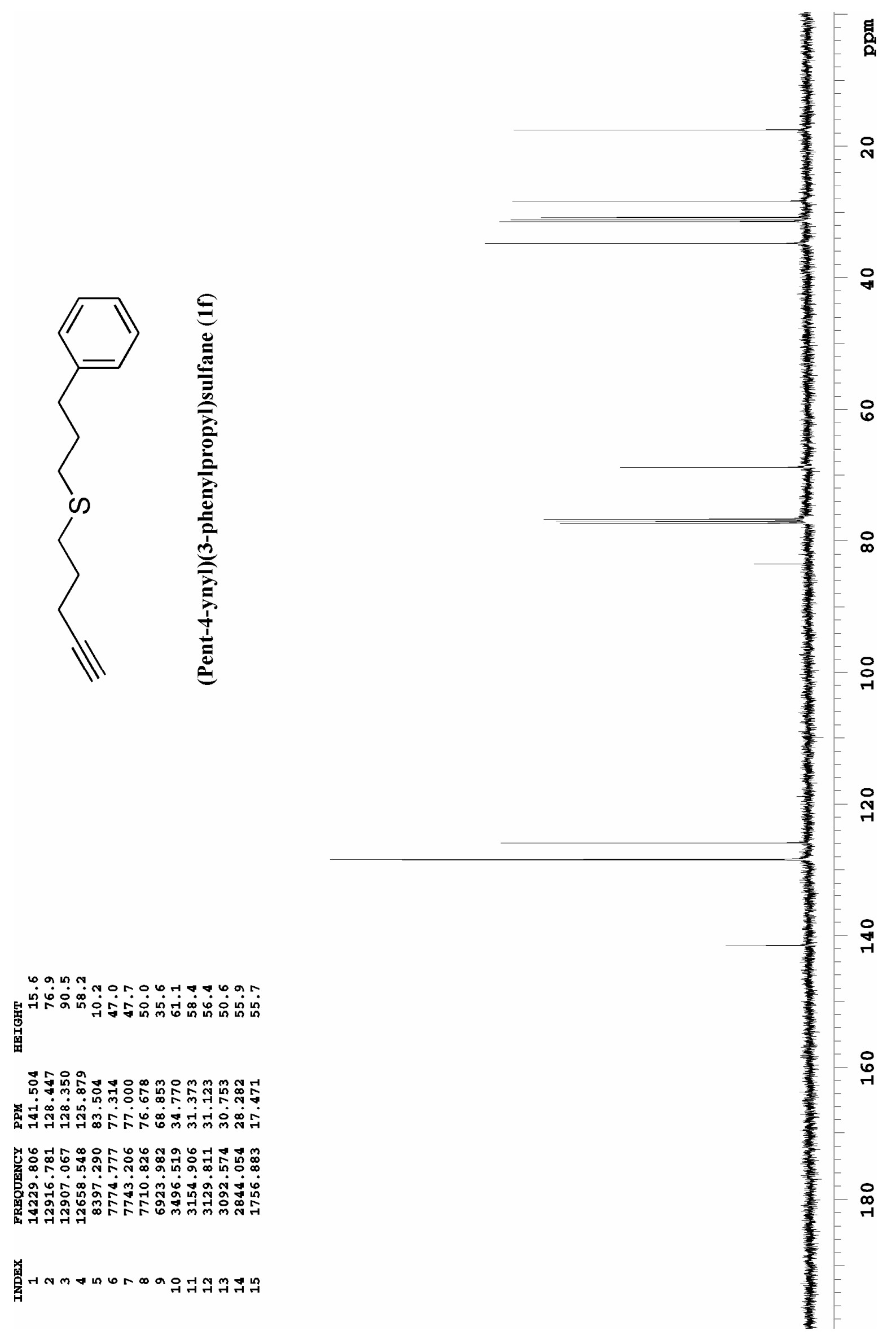




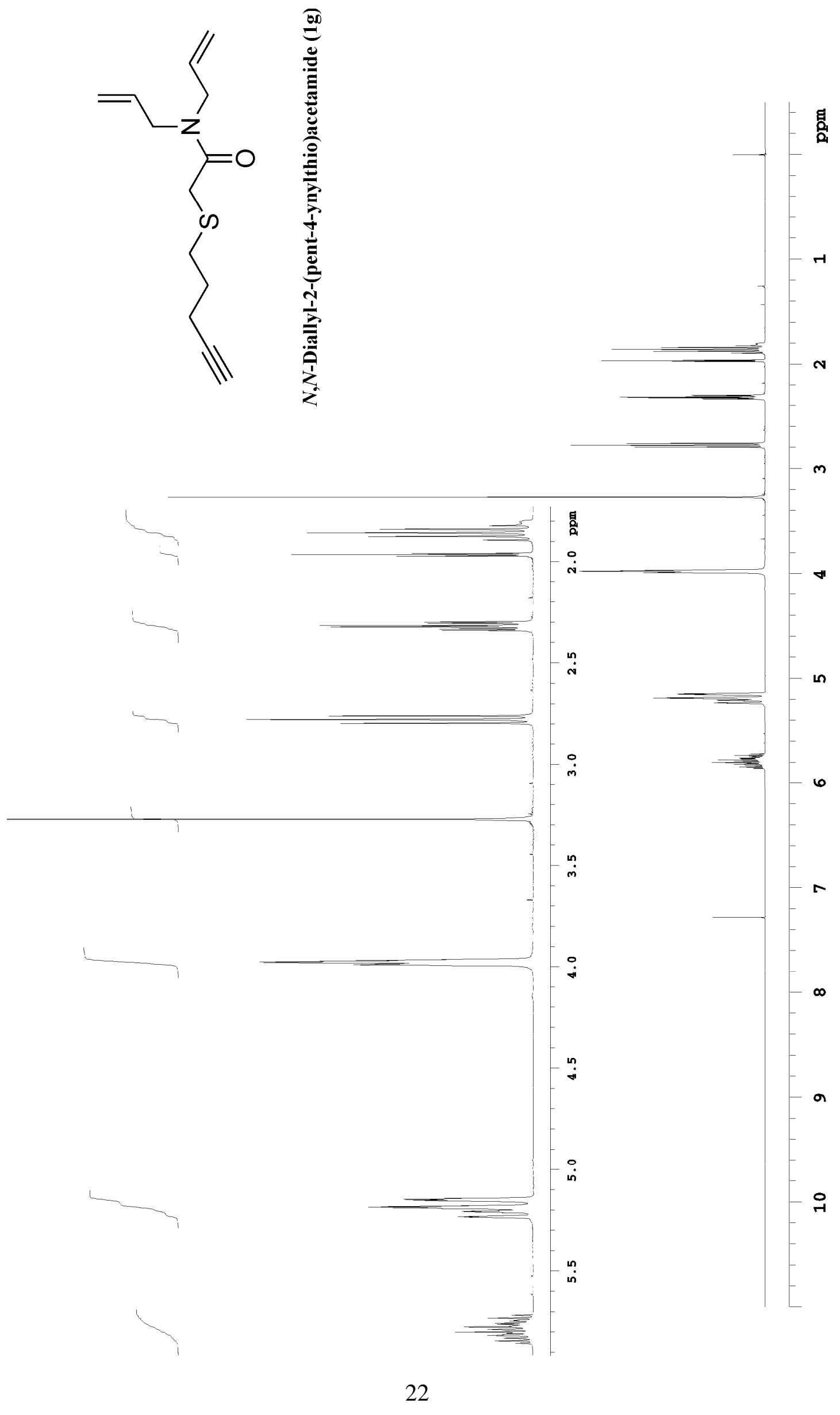



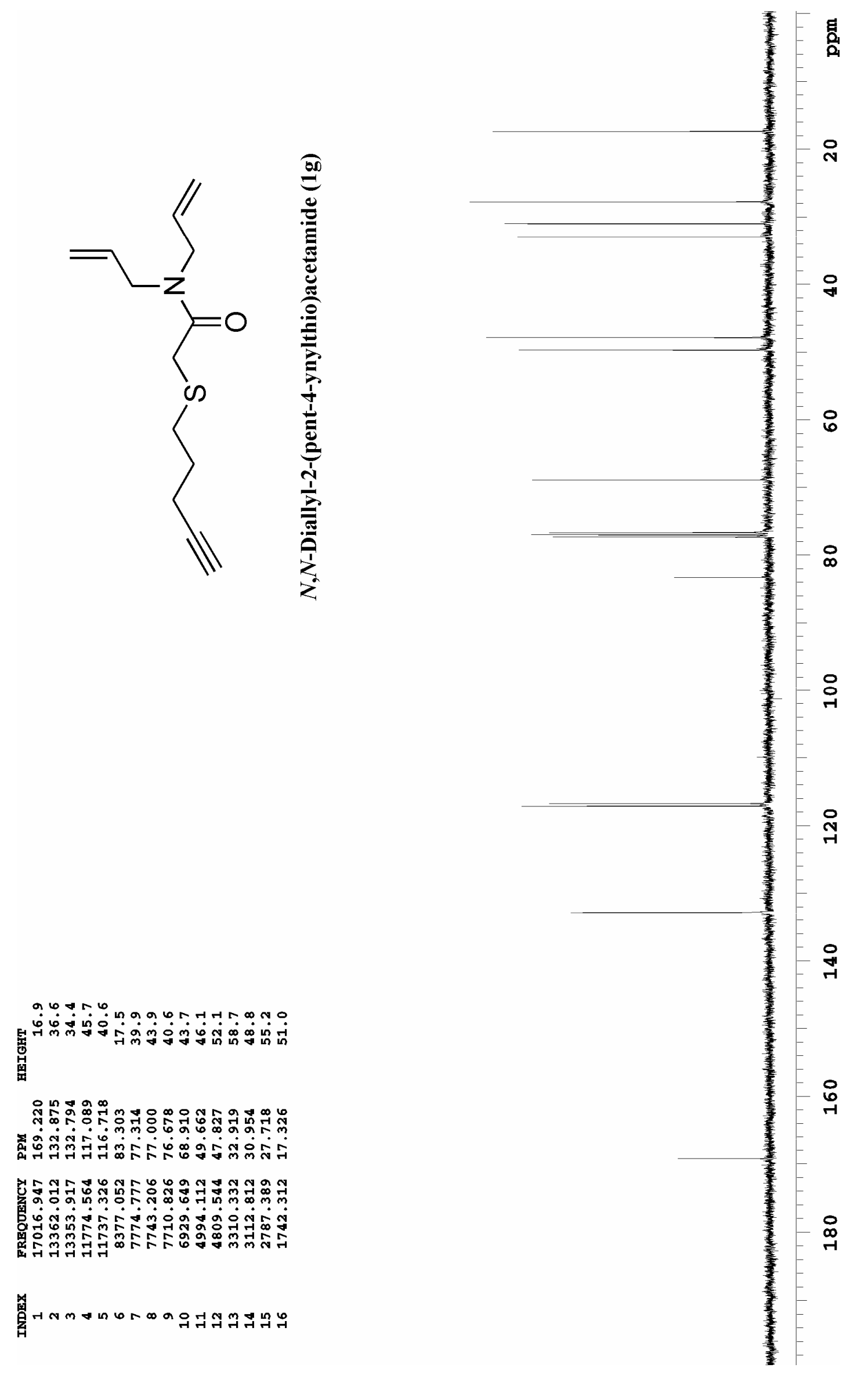


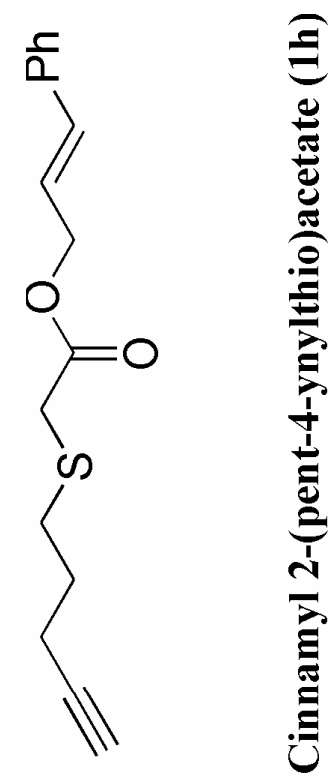

בُ

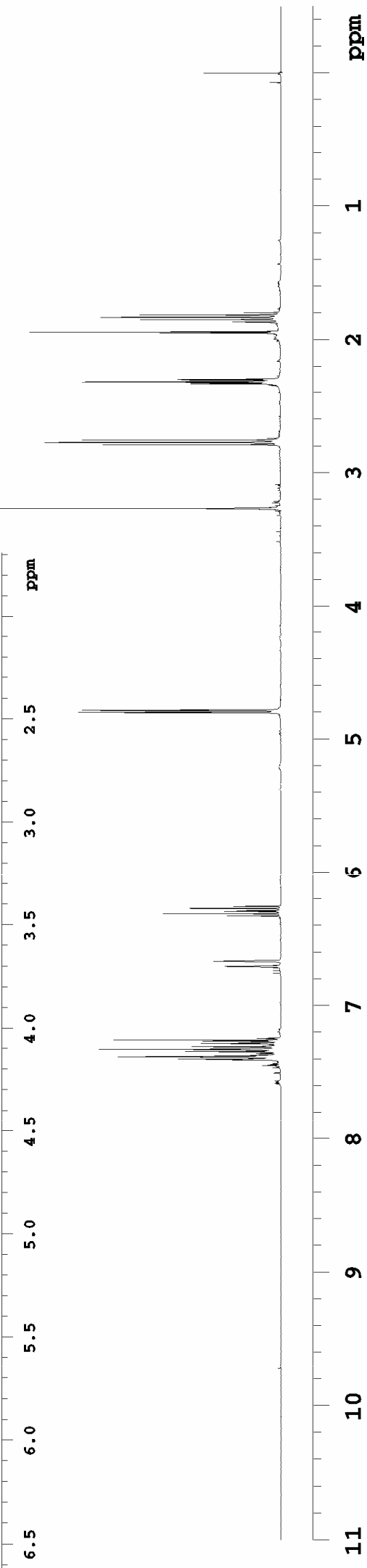




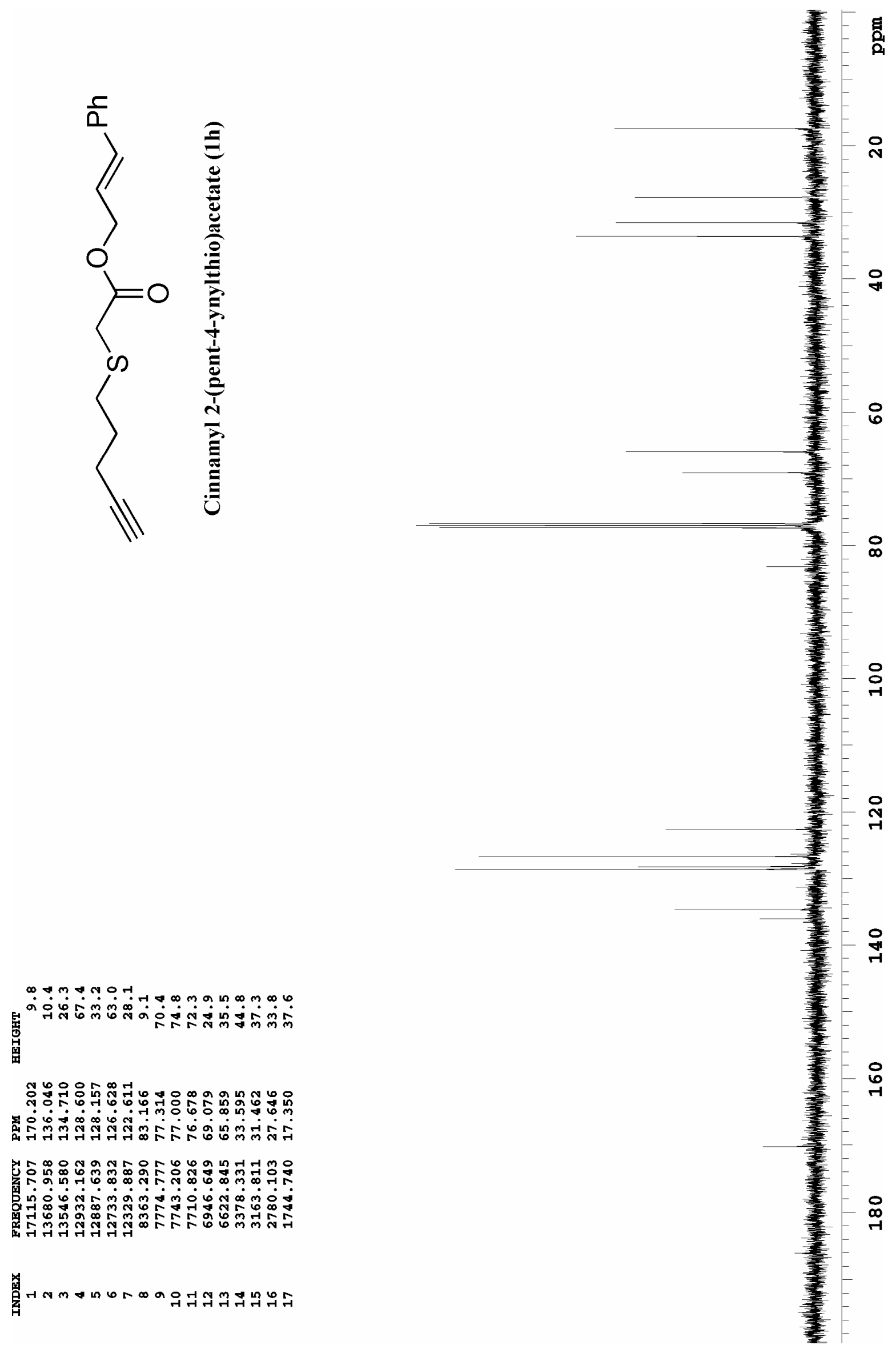




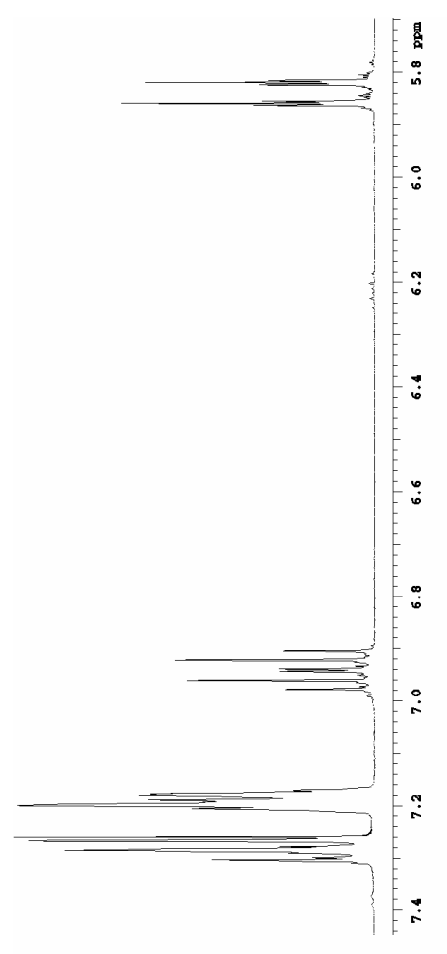

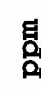

N
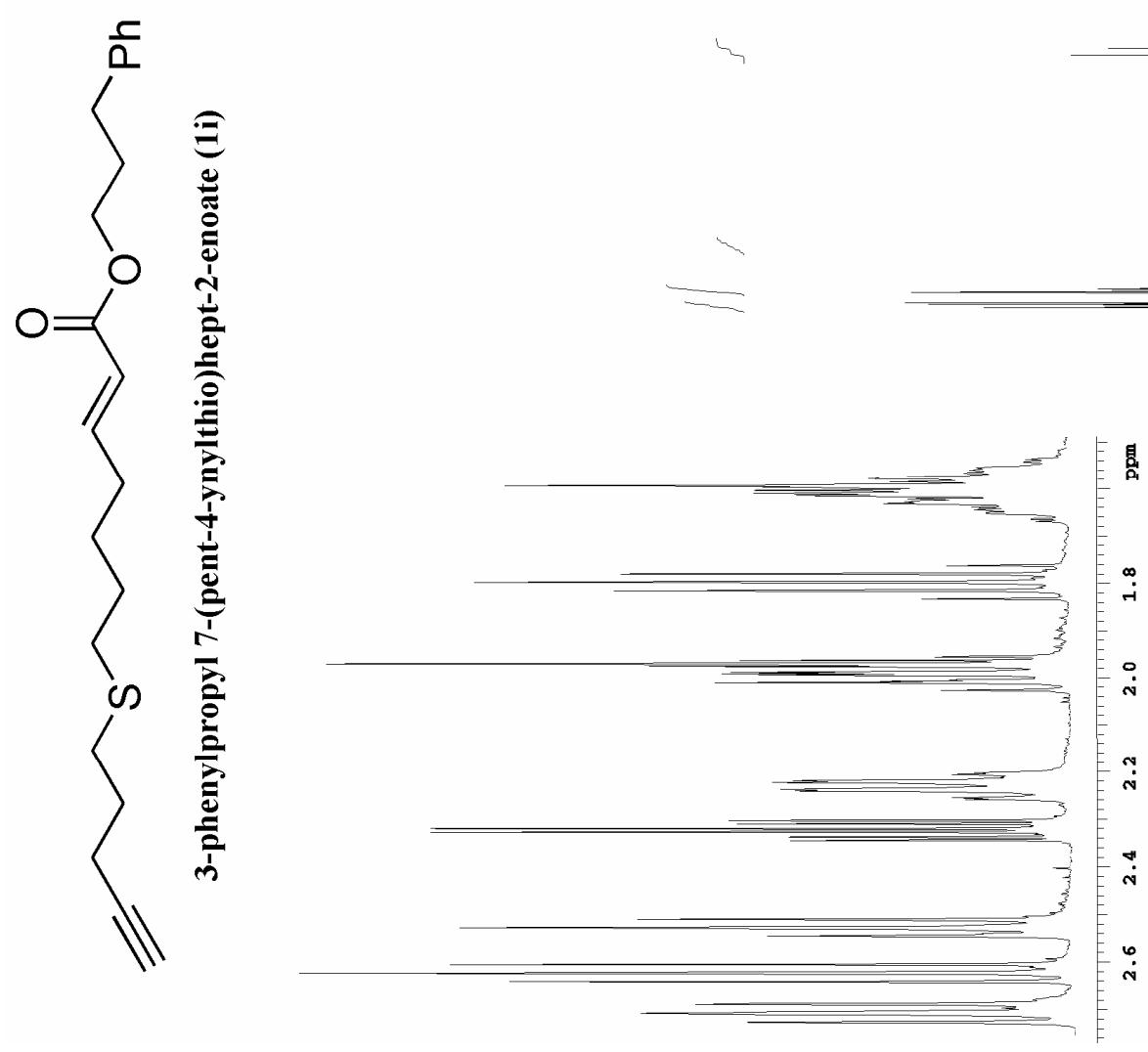

$\infty$

a

우 


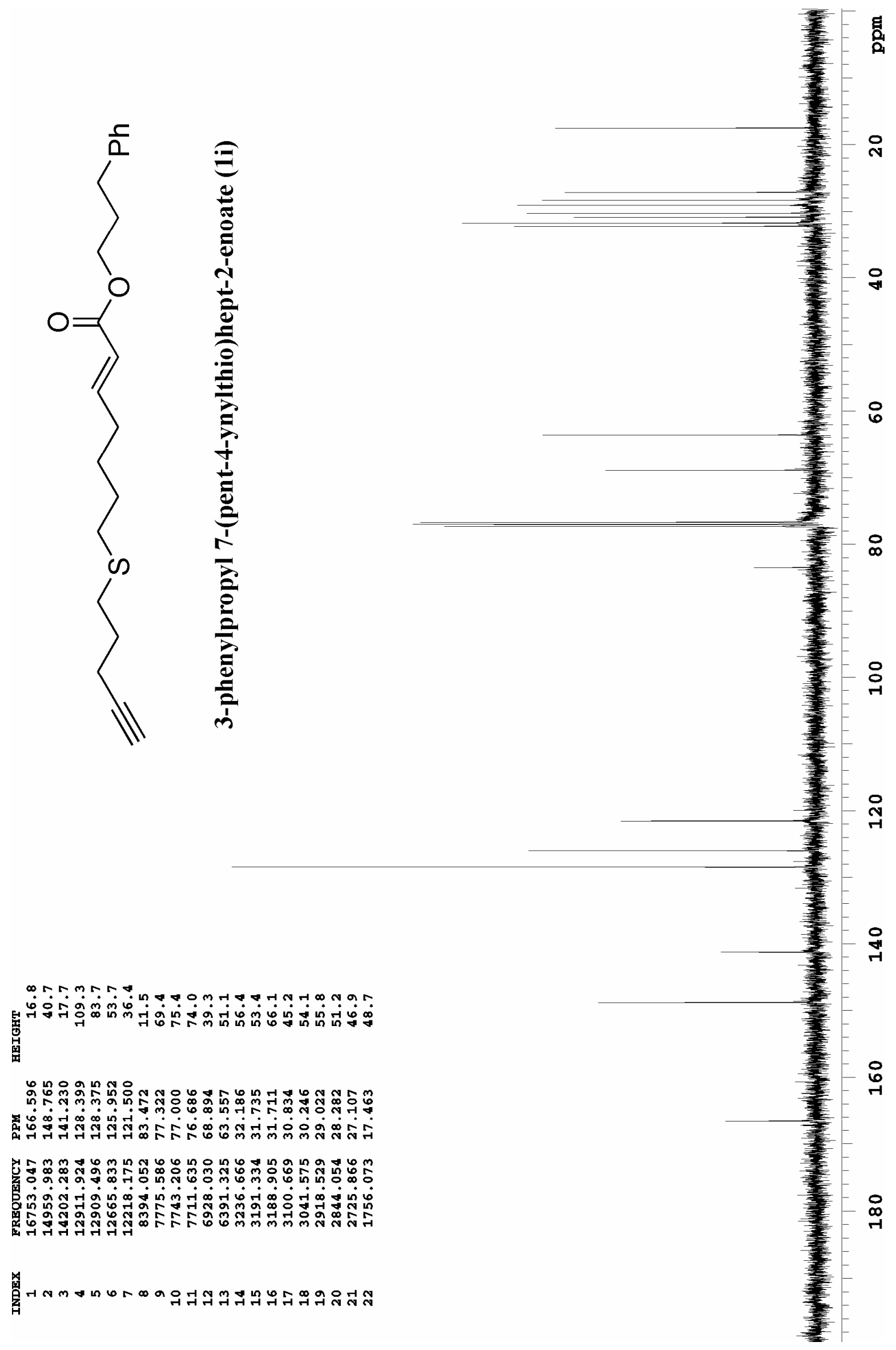




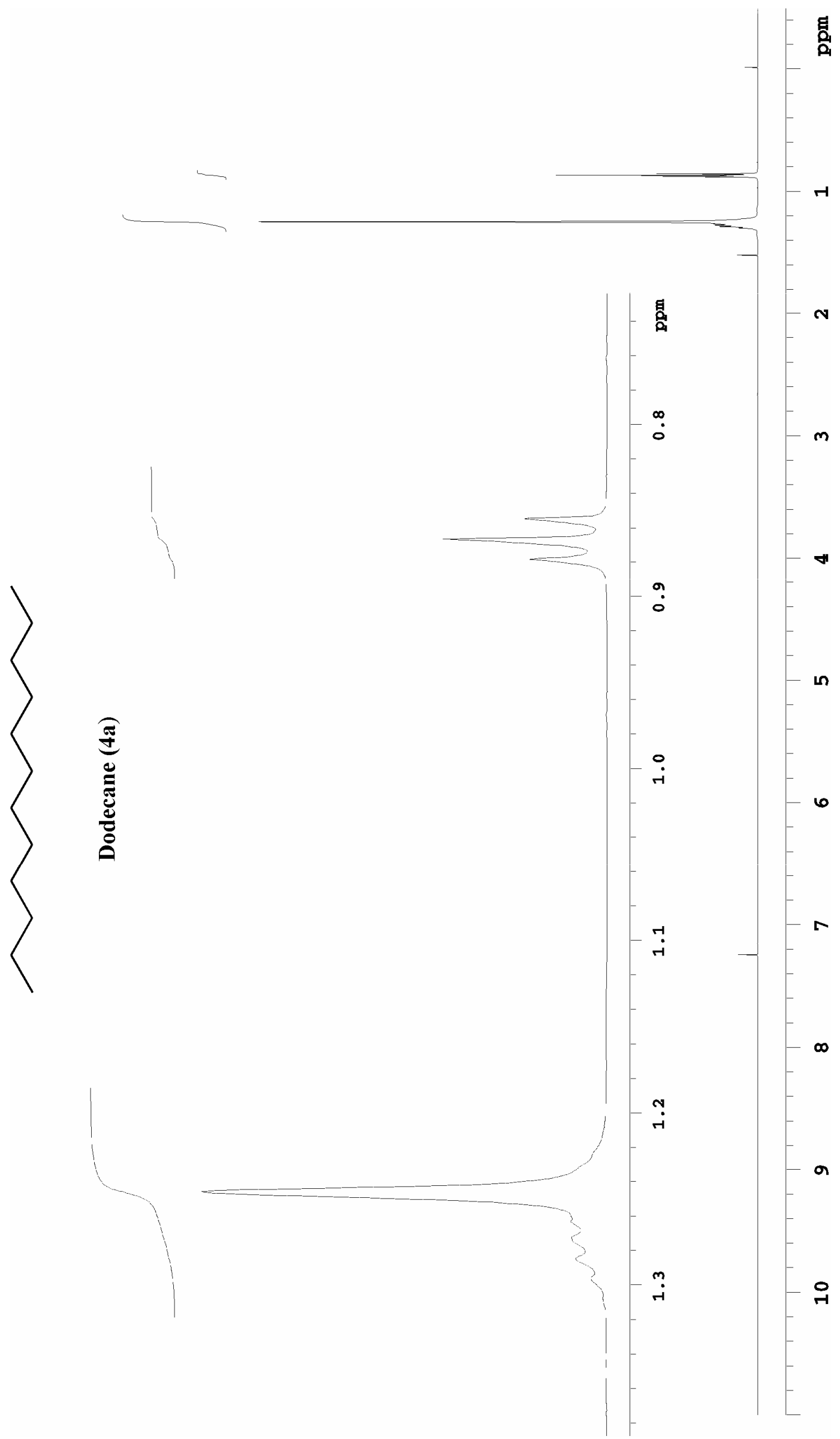




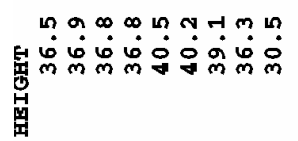

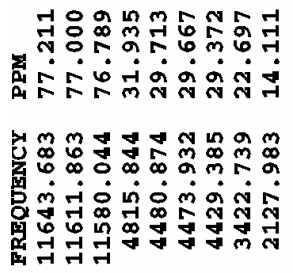

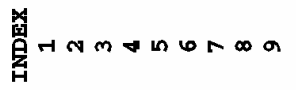

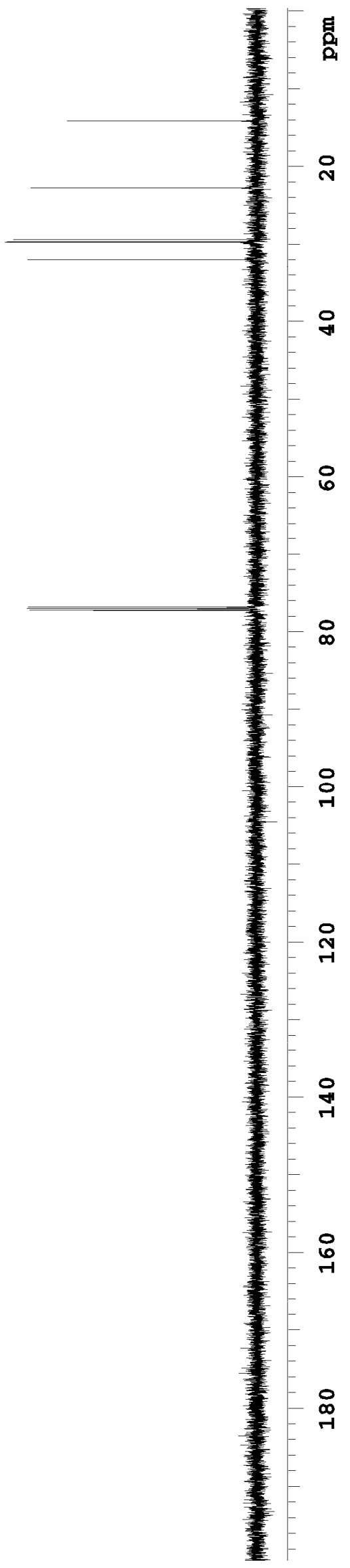

29 


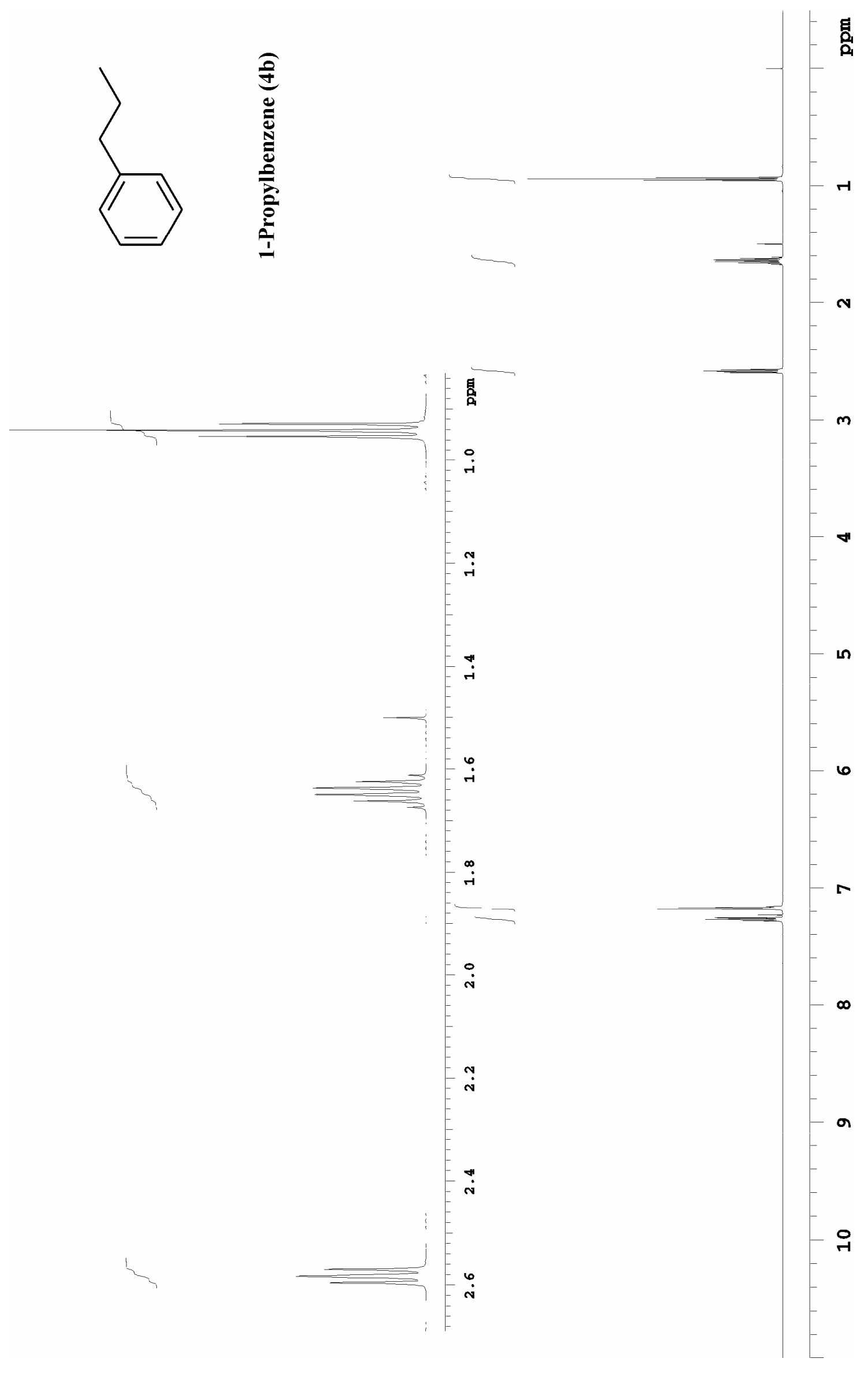




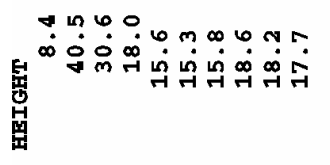

눈

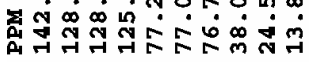

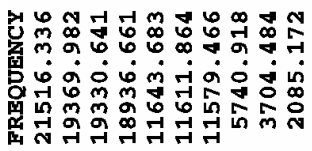

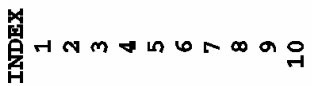
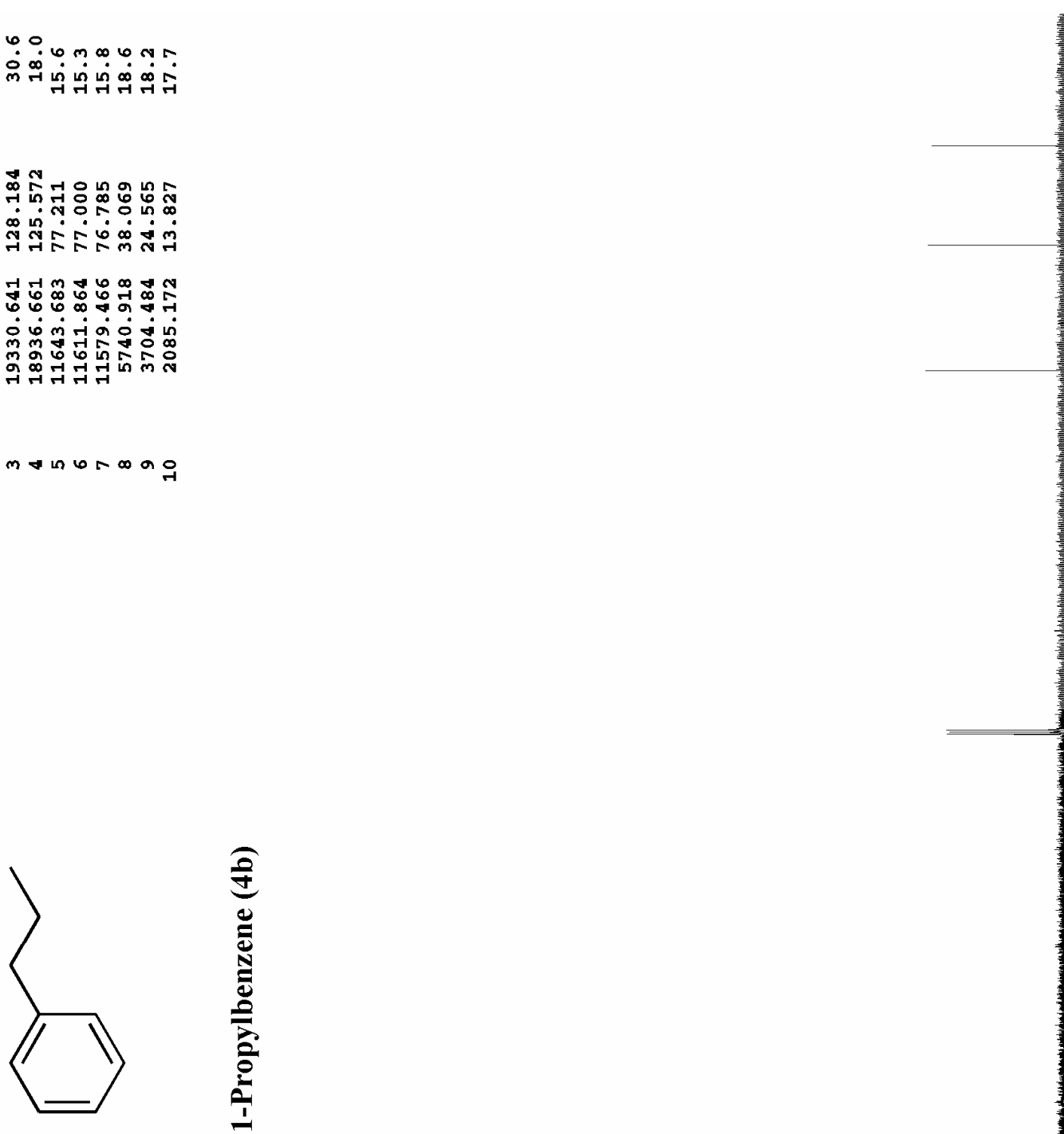

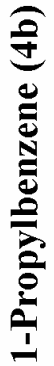




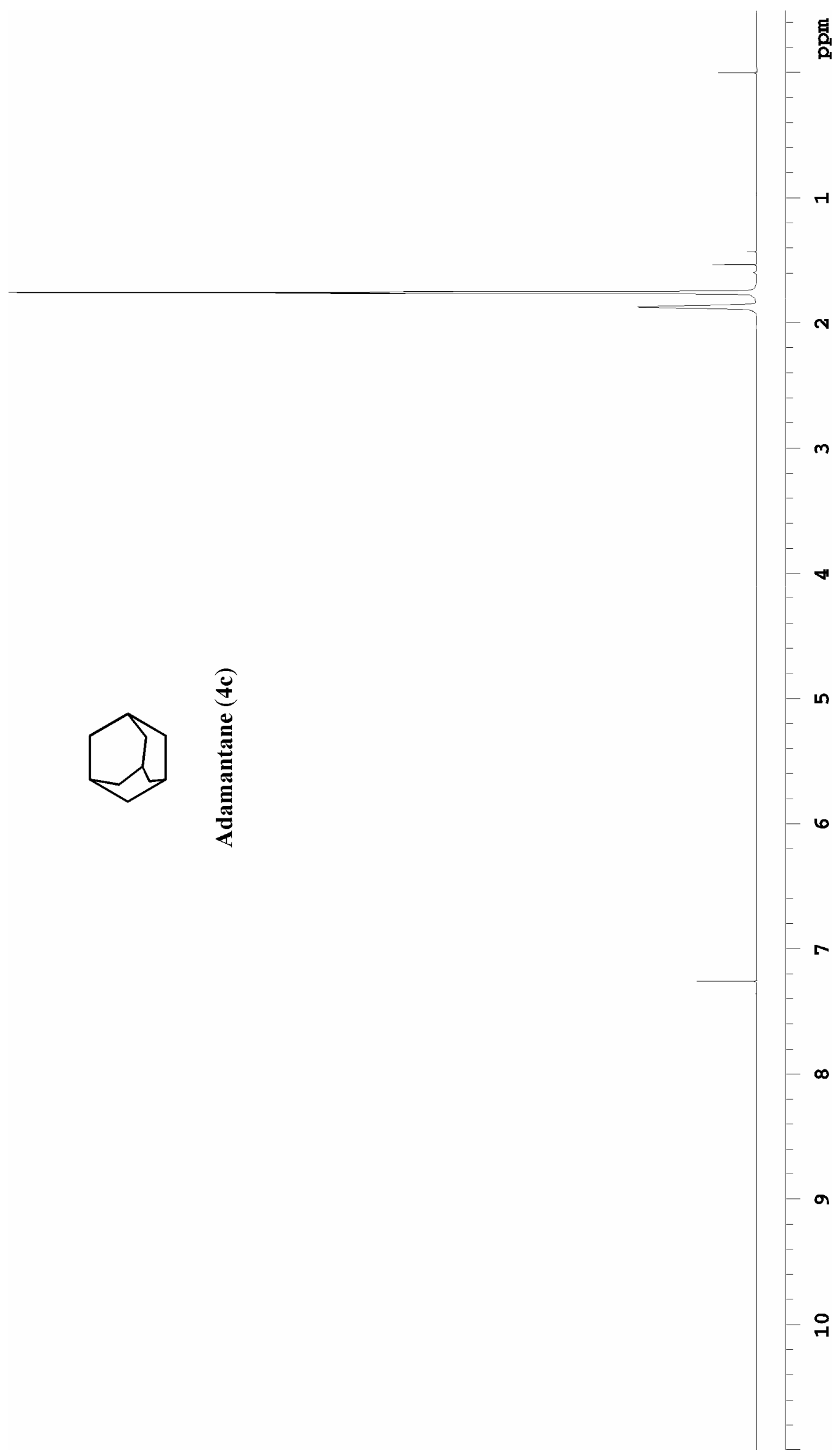




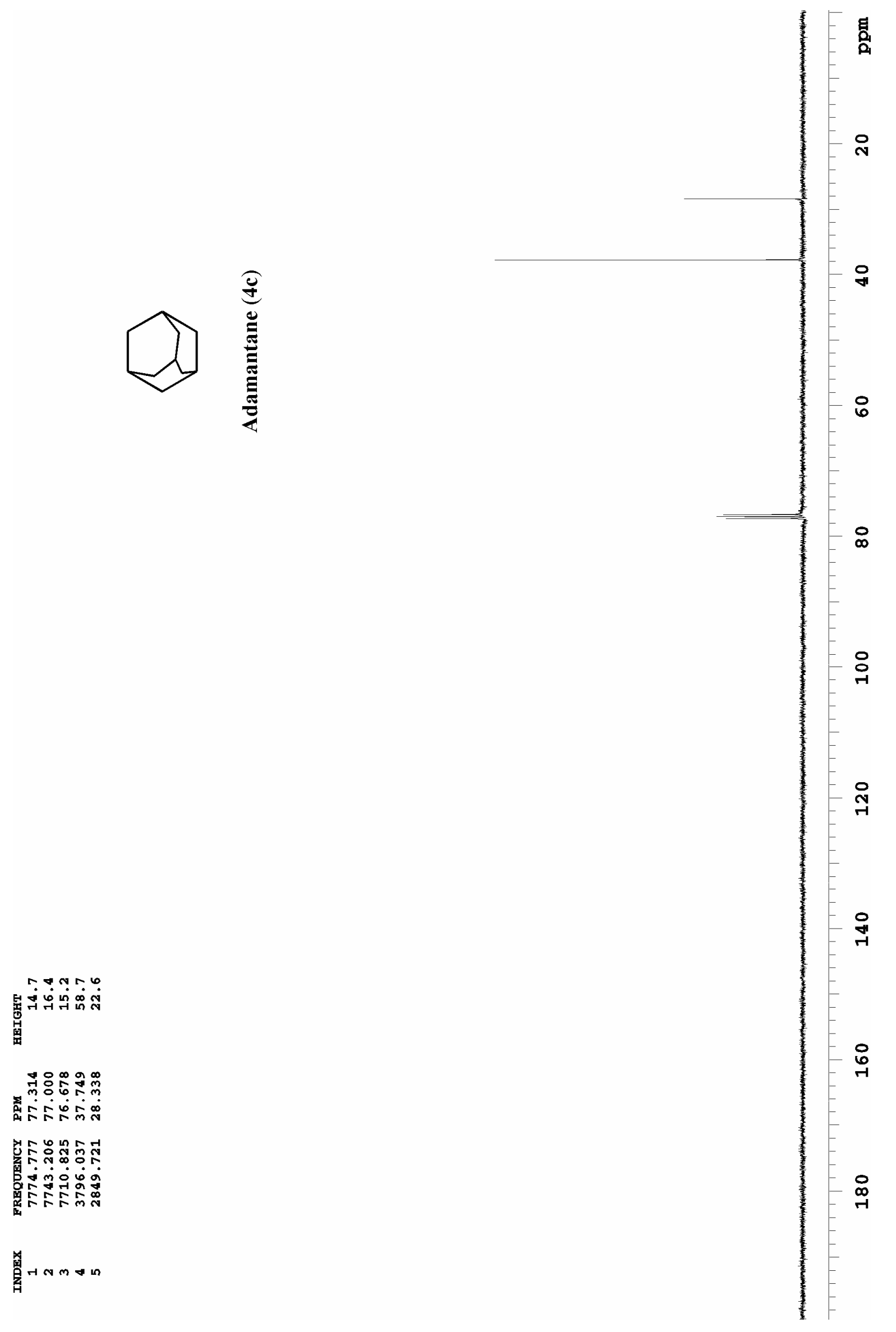




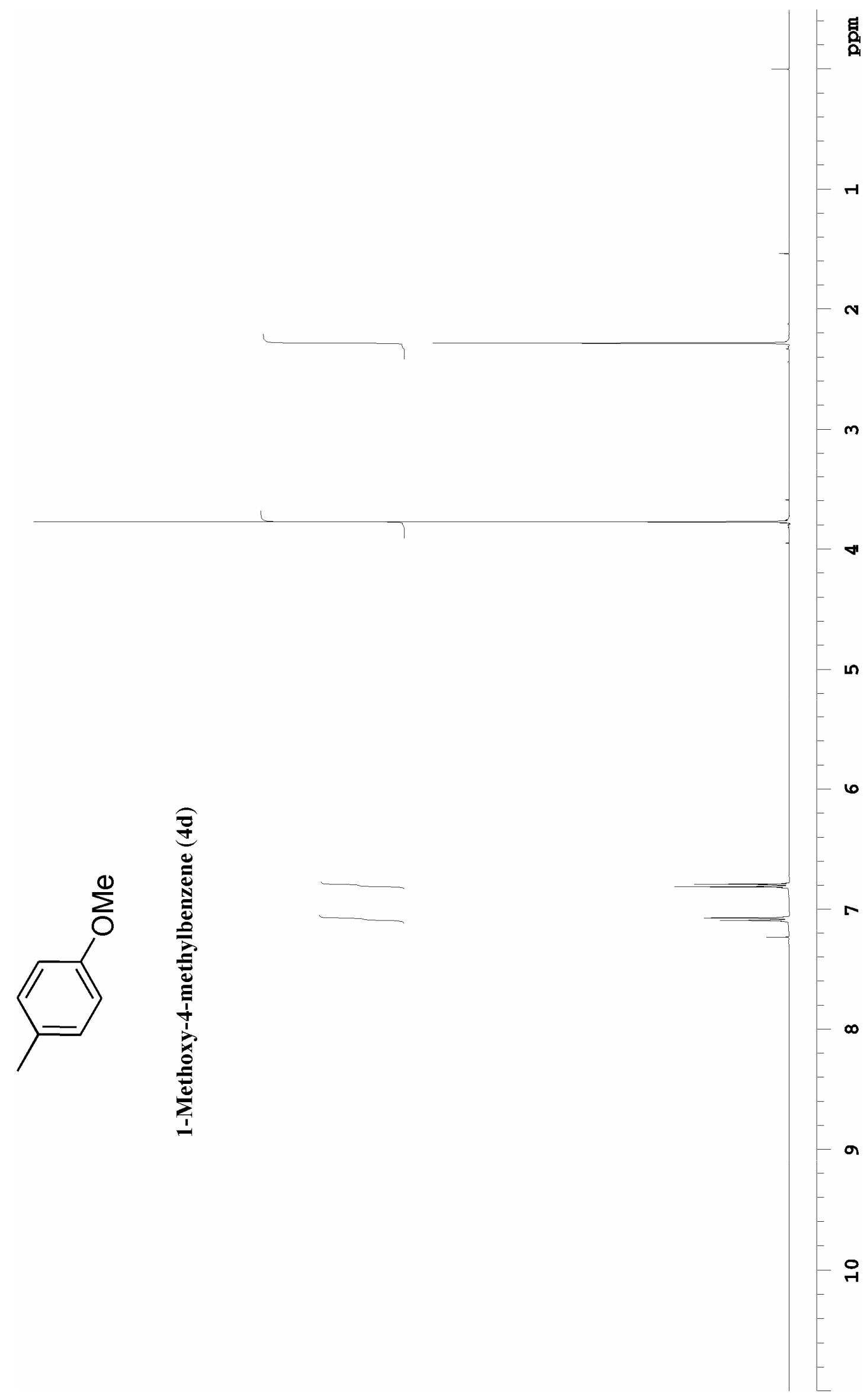




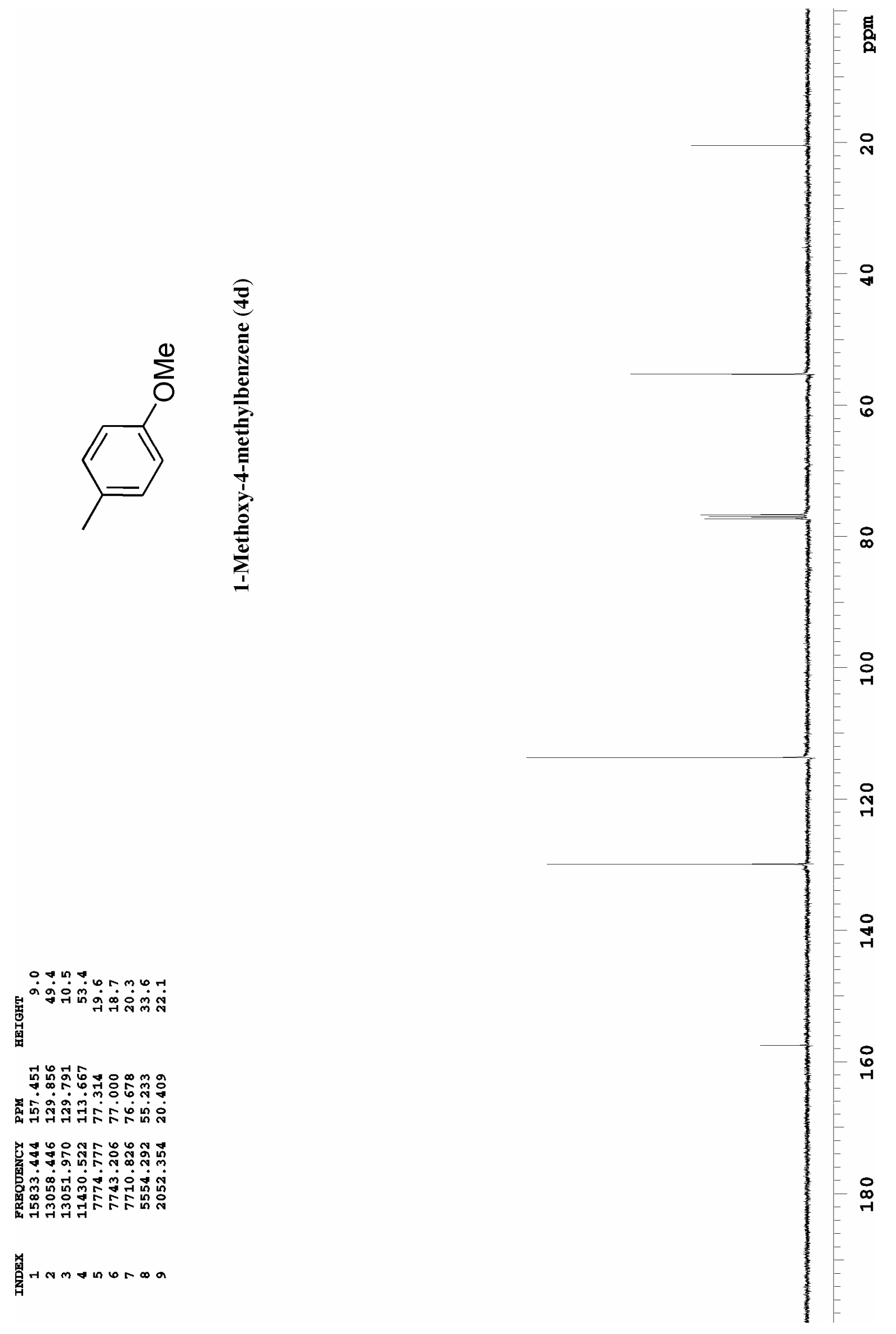




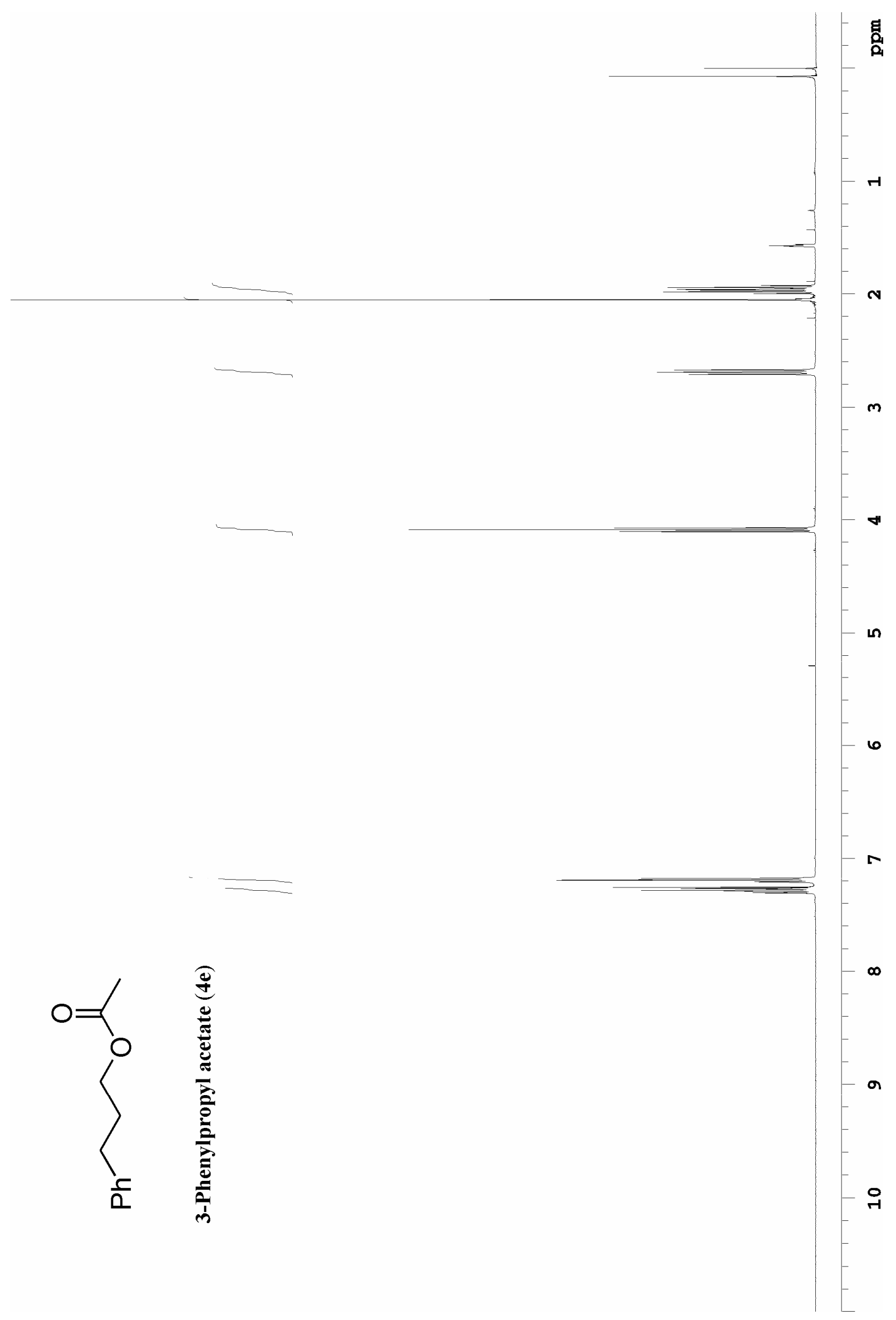




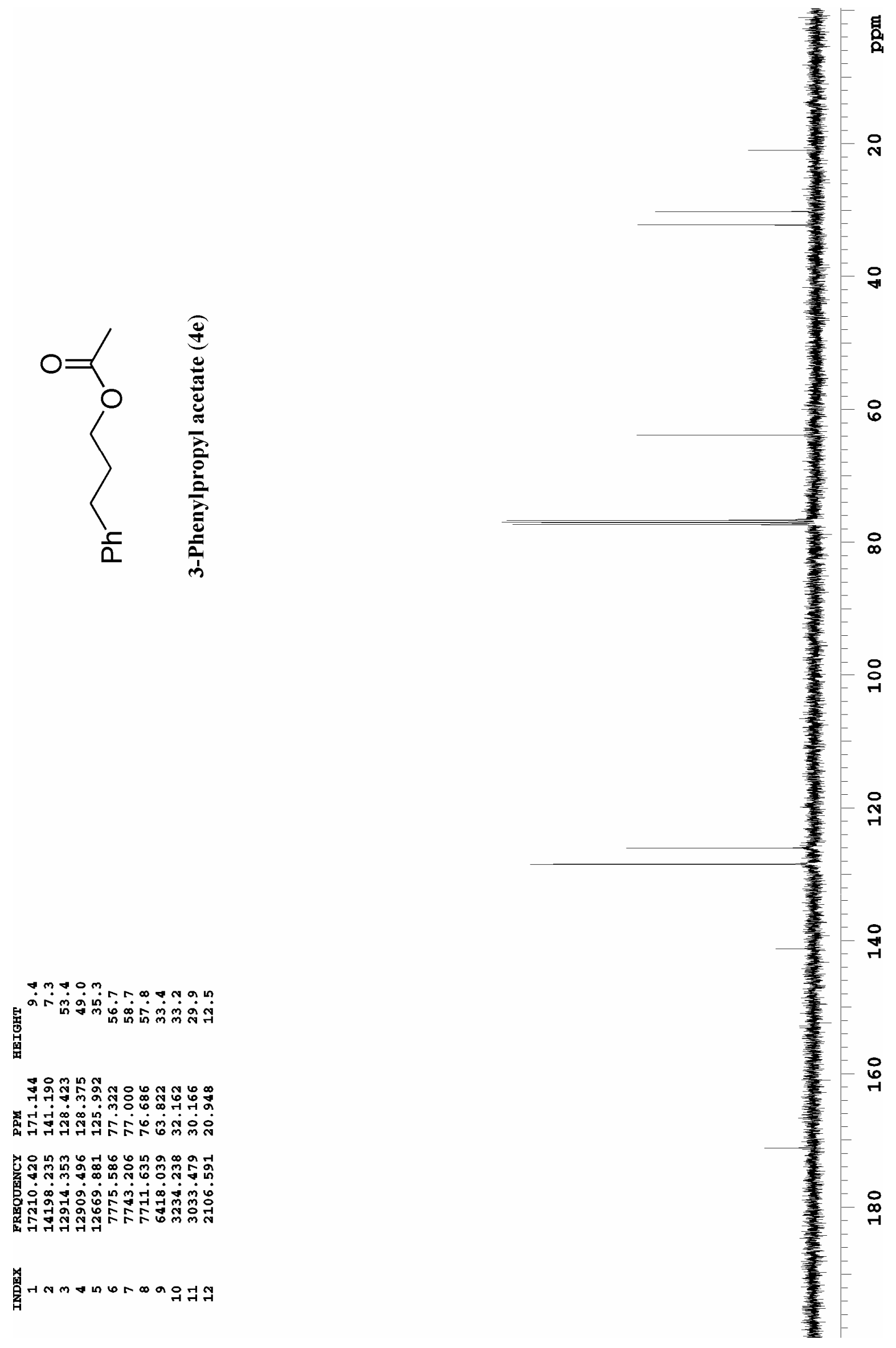




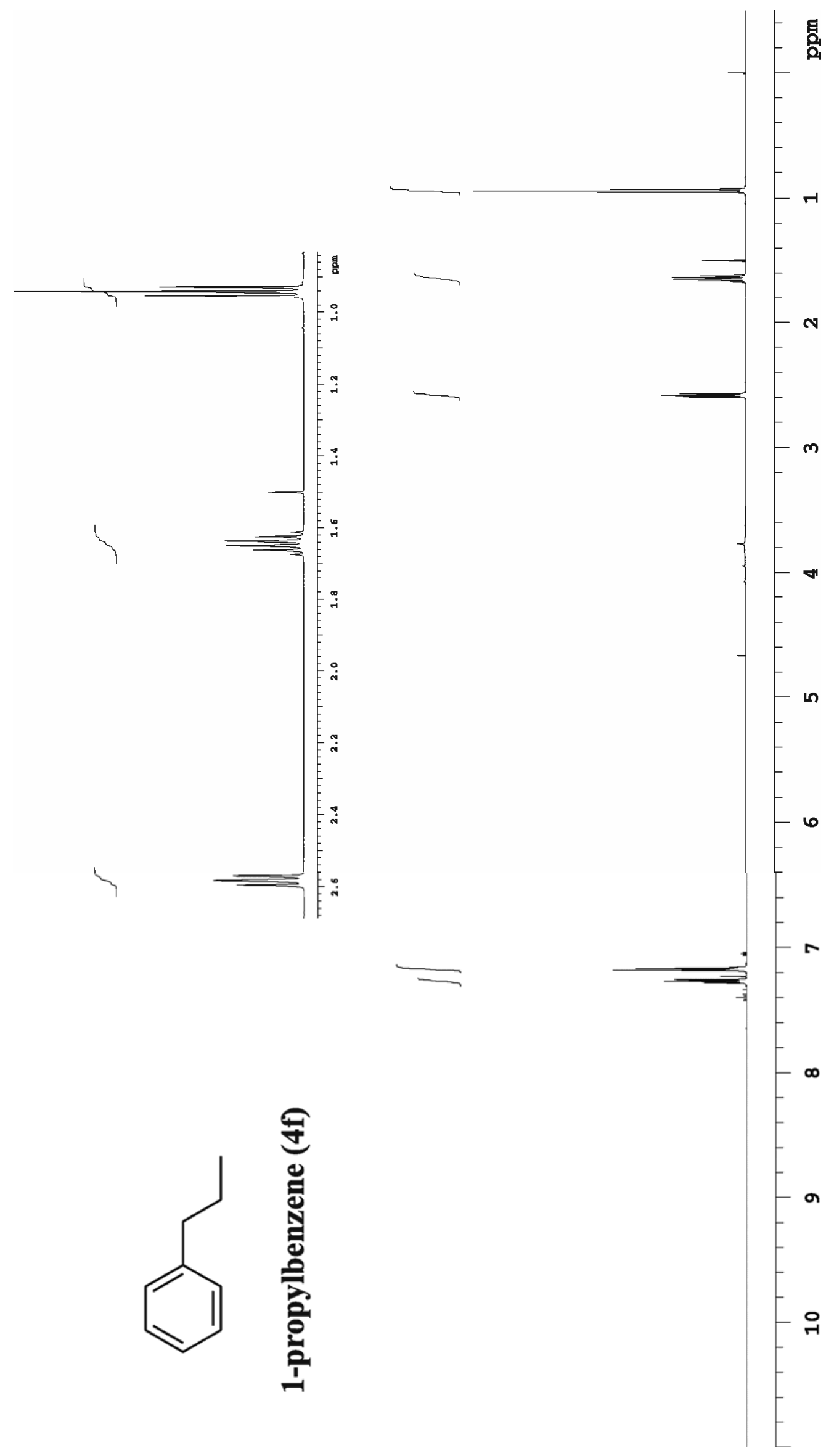



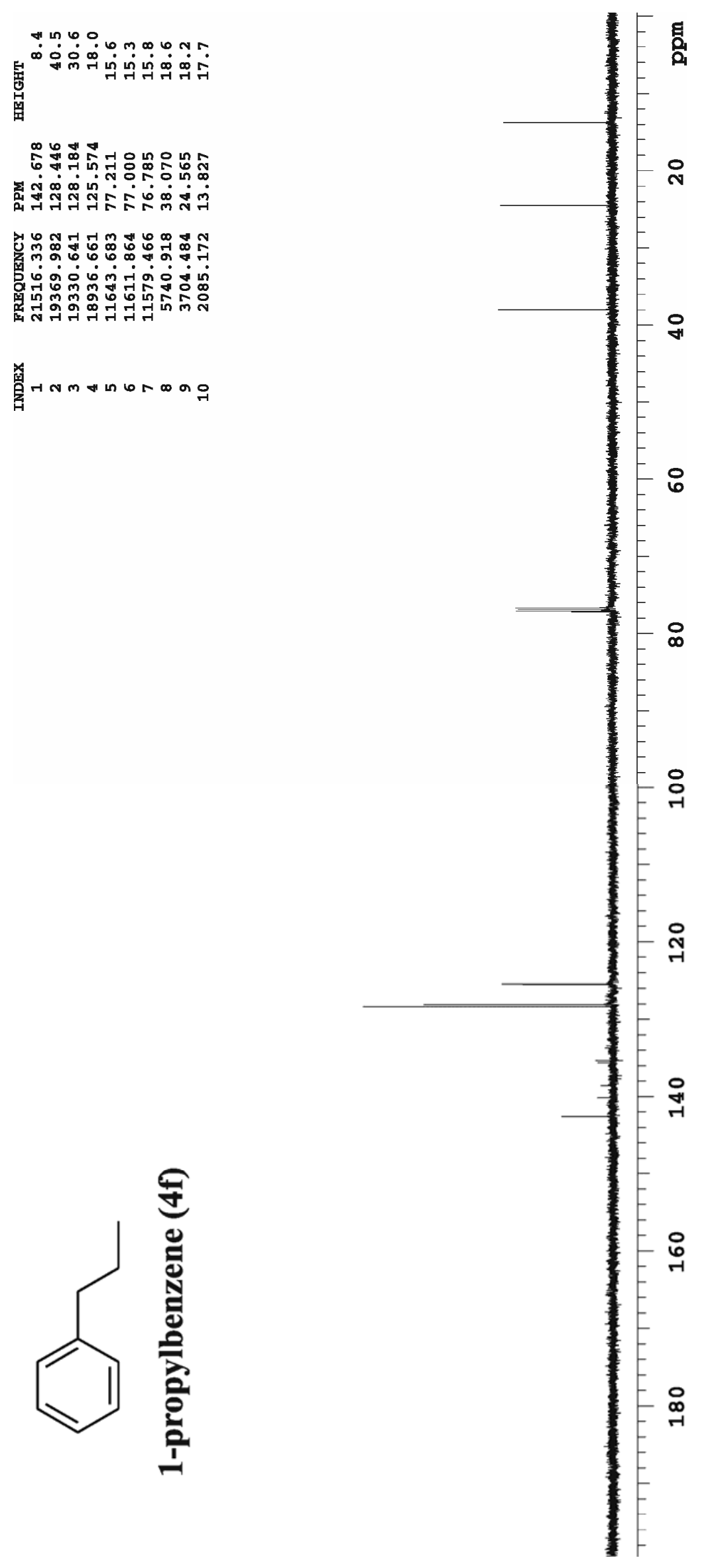


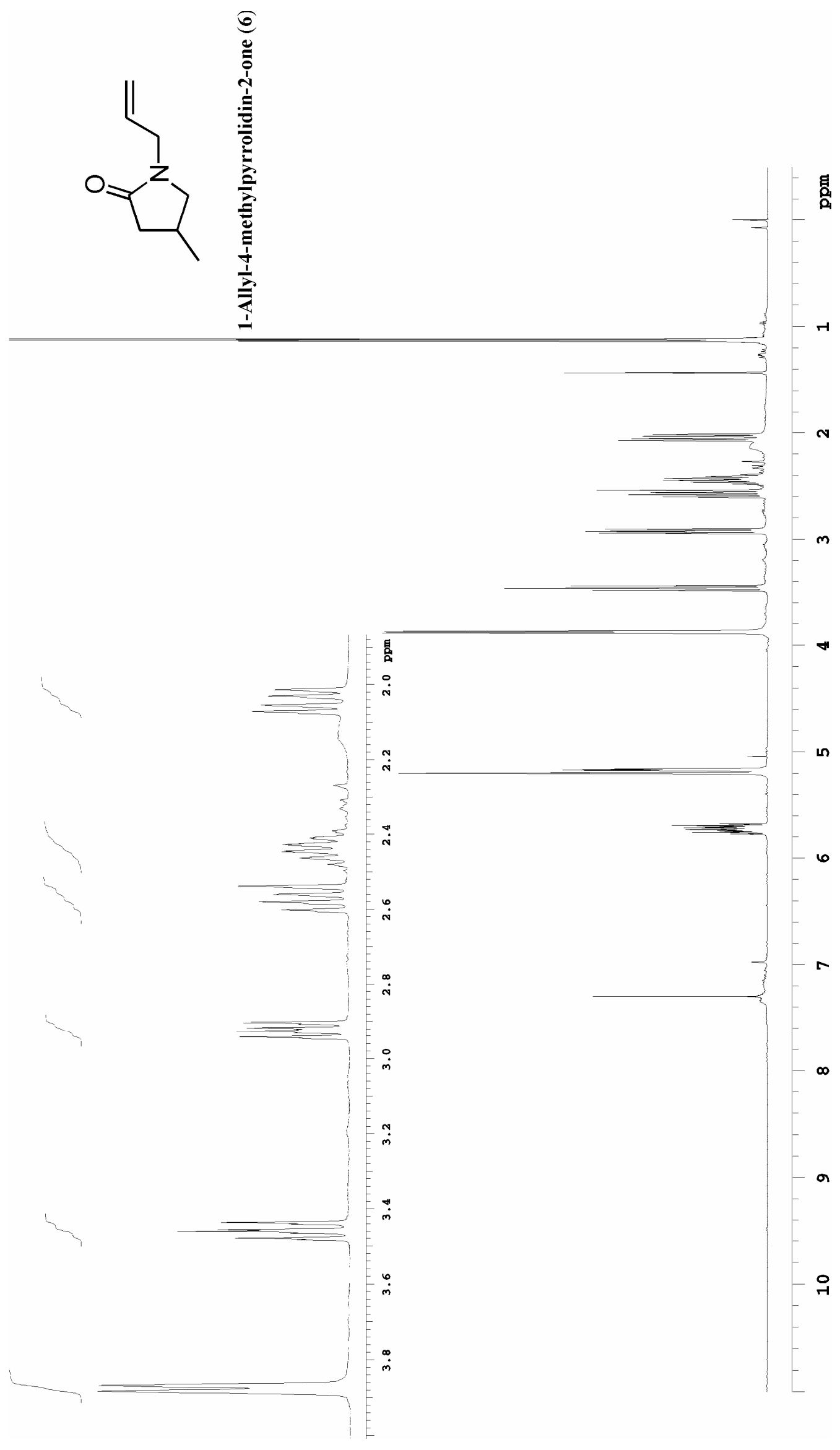




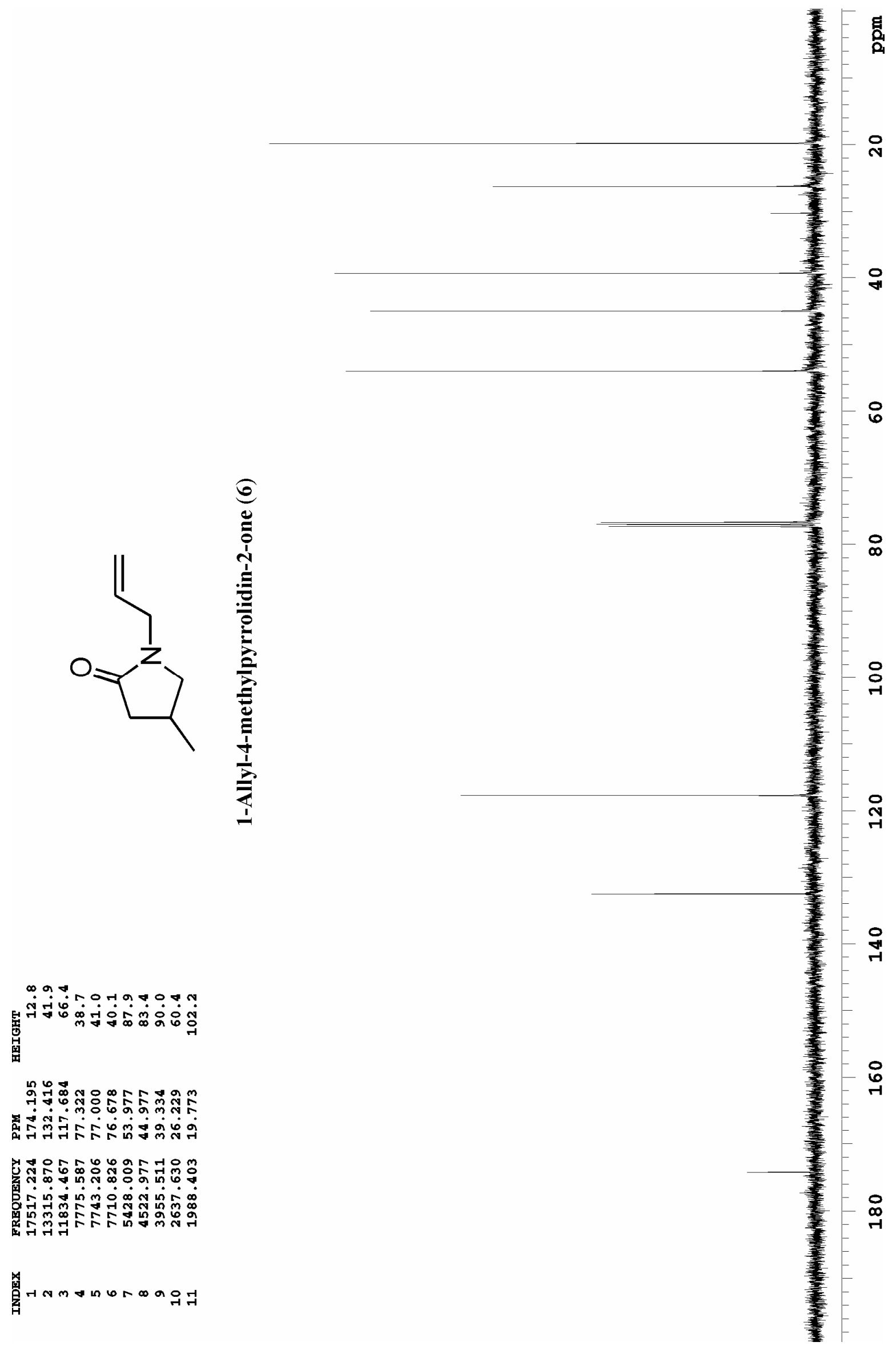




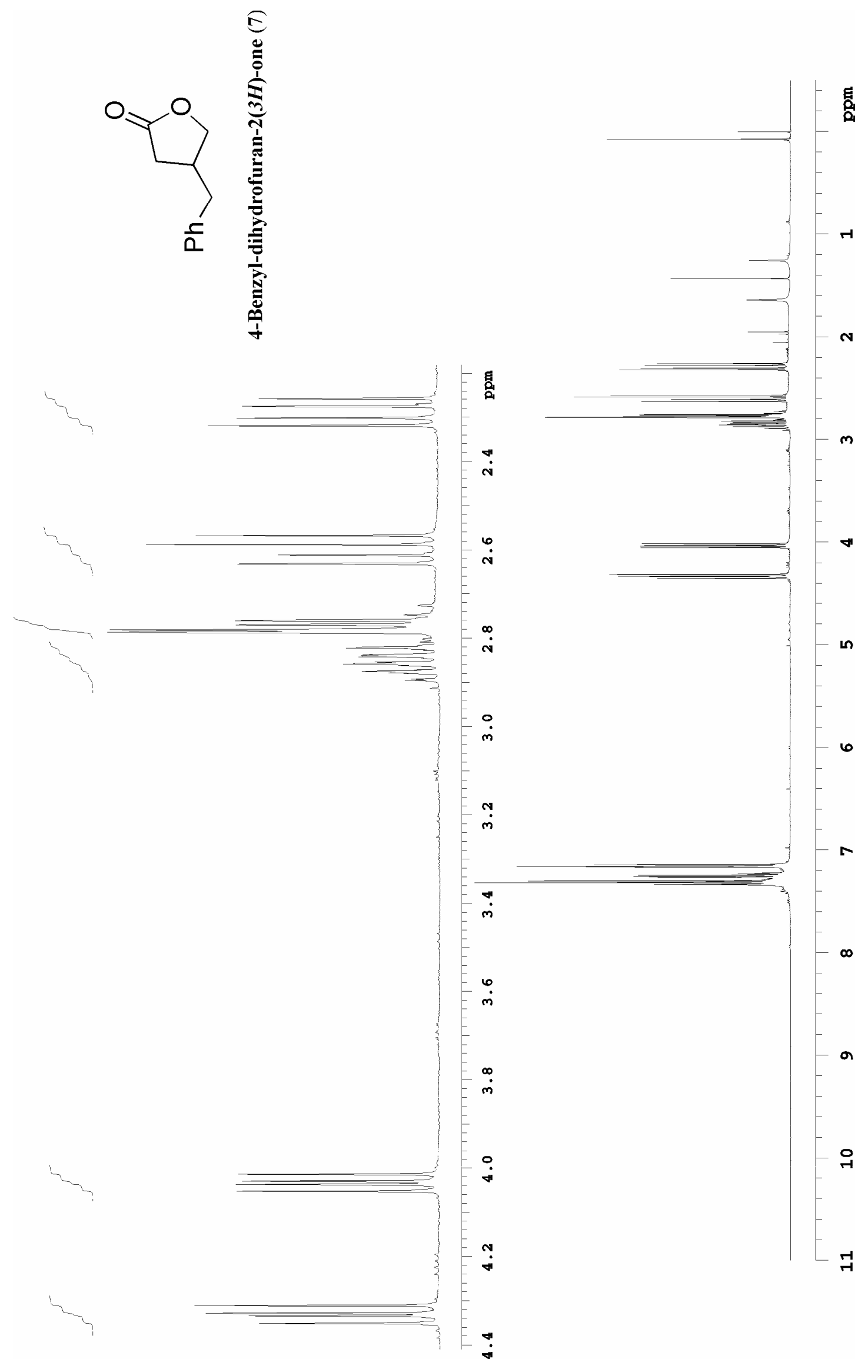




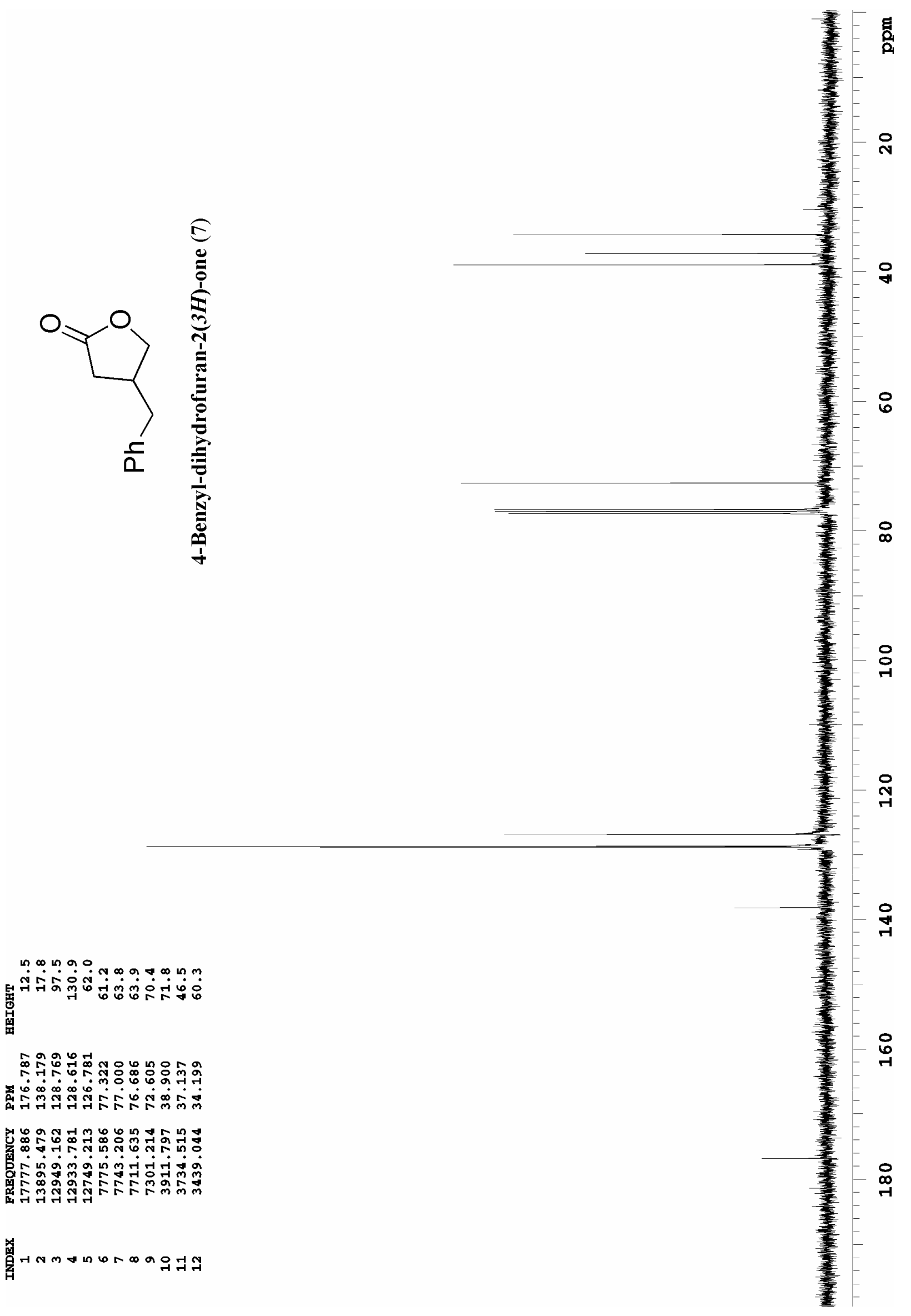




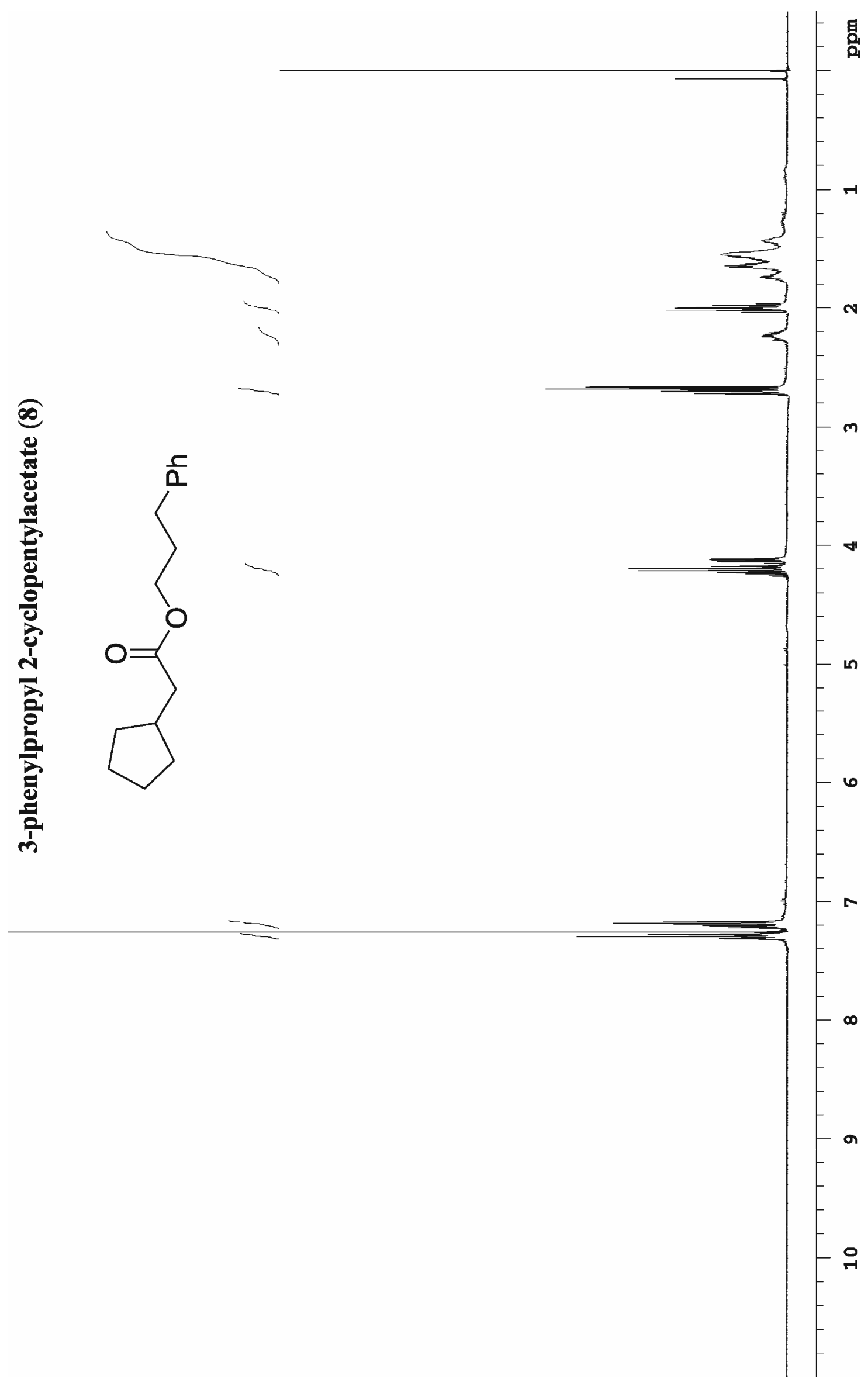




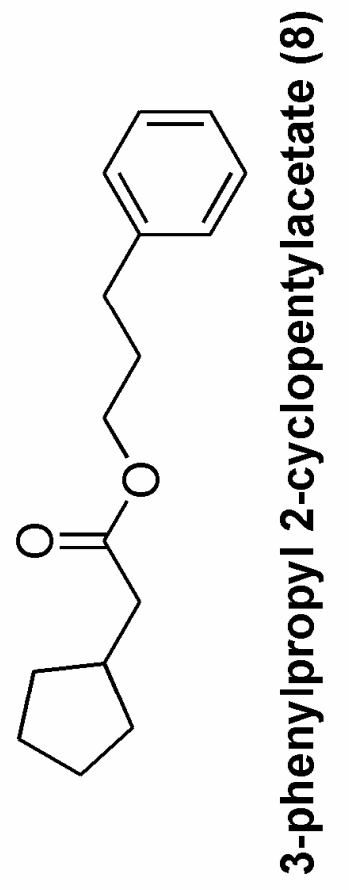

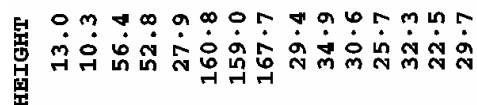

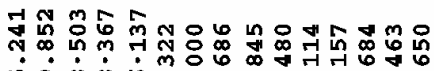

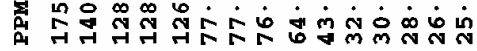

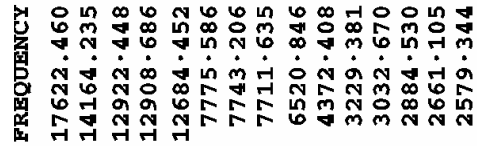

葸 


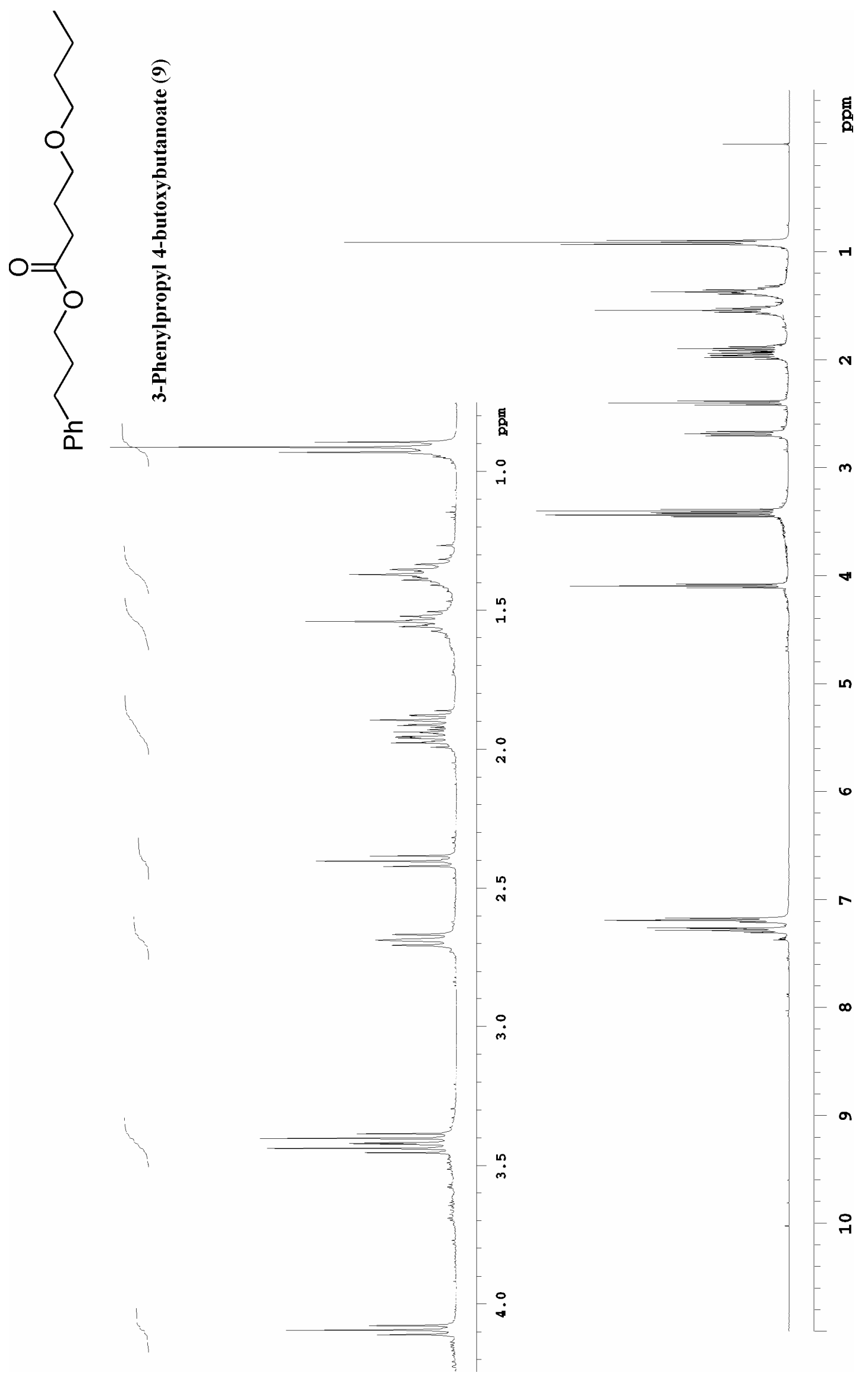




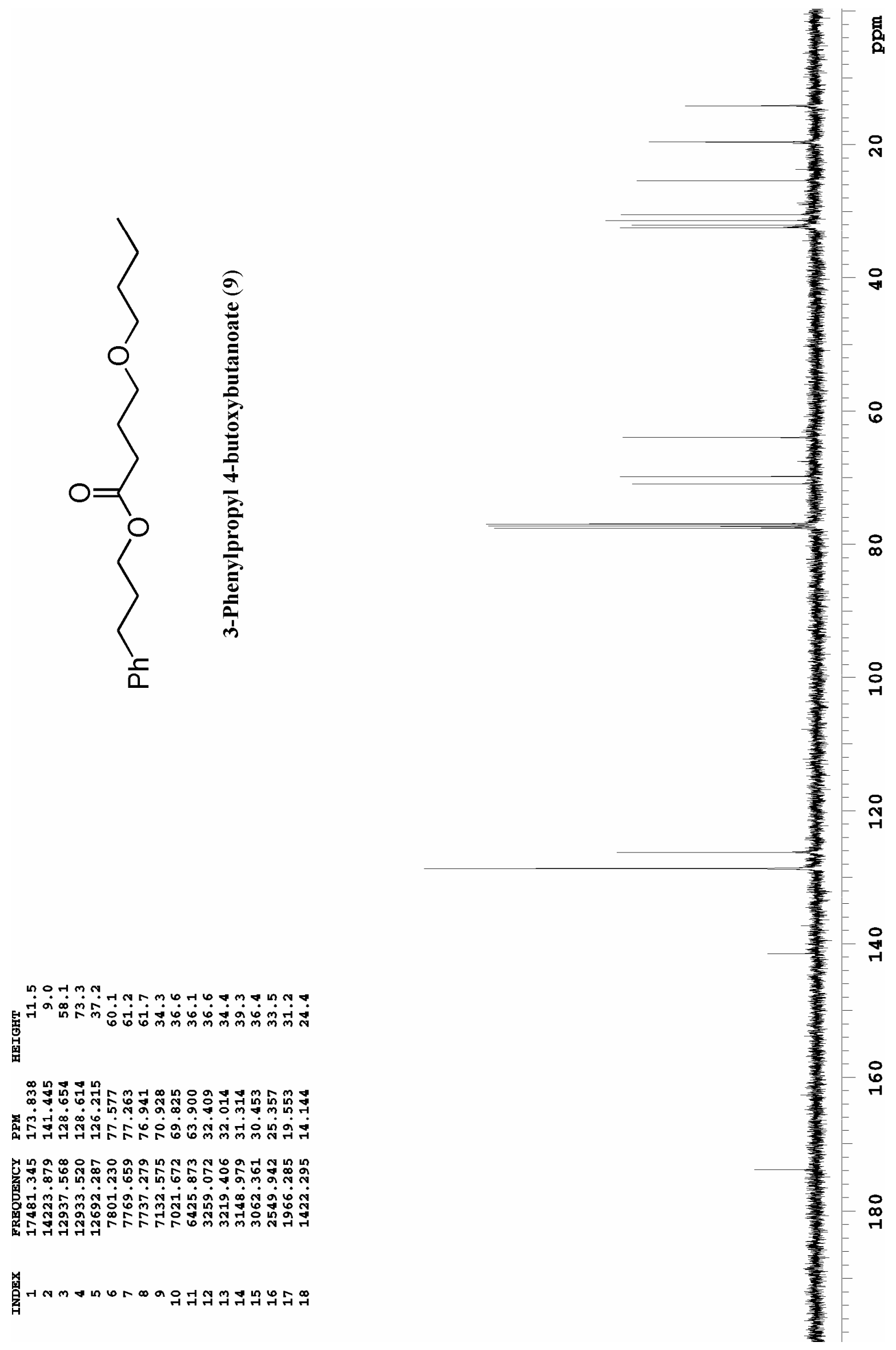




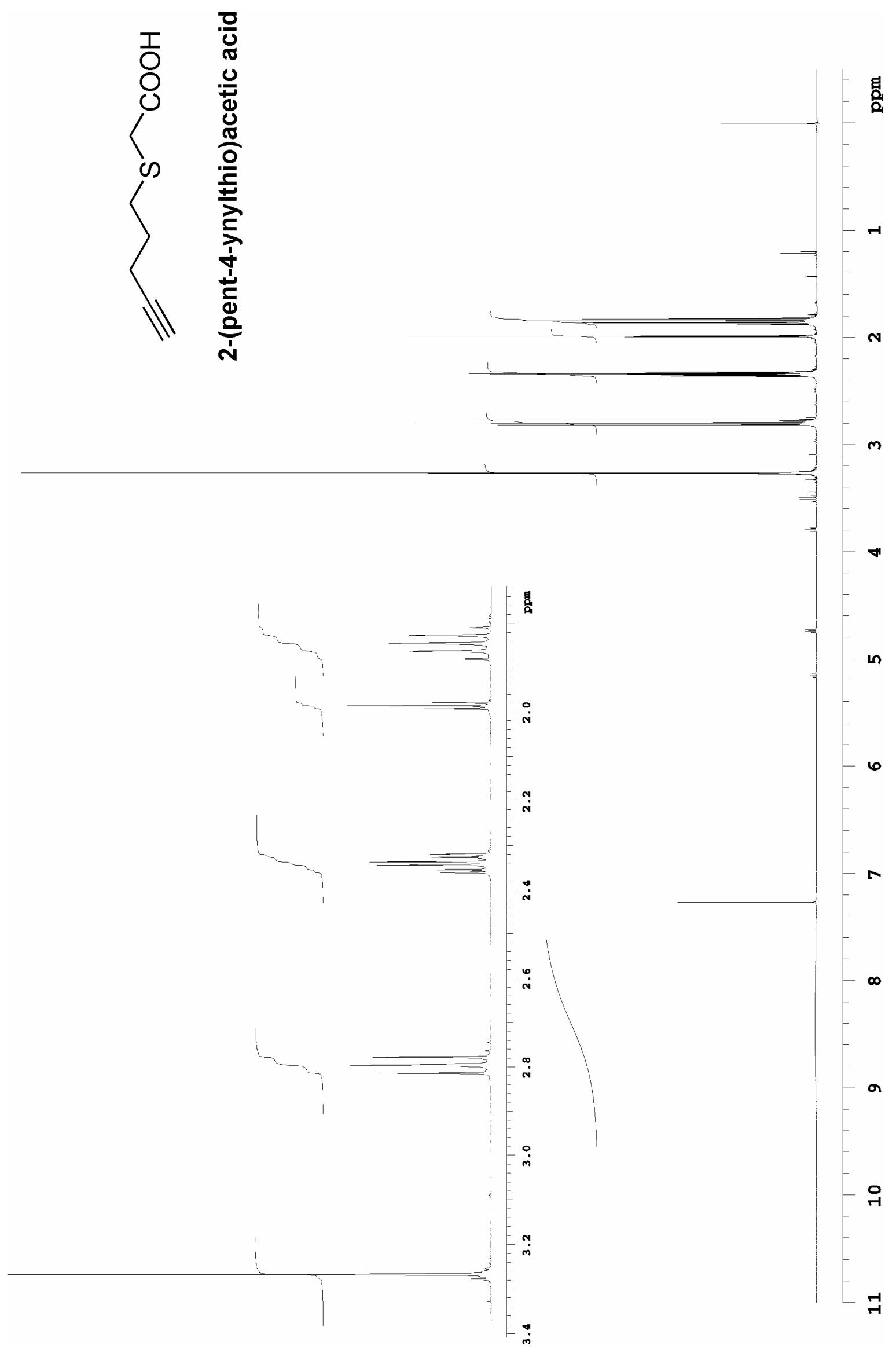




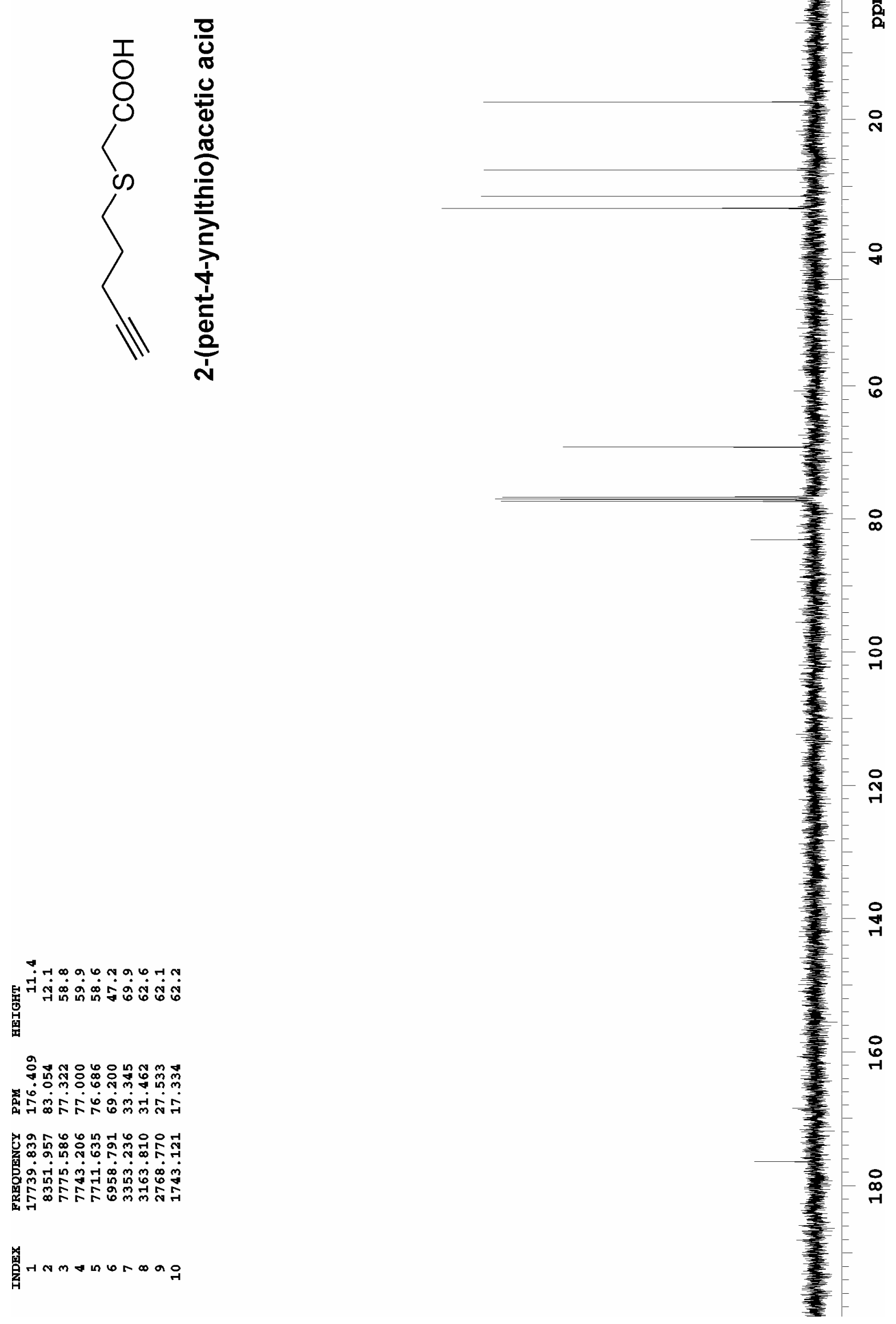




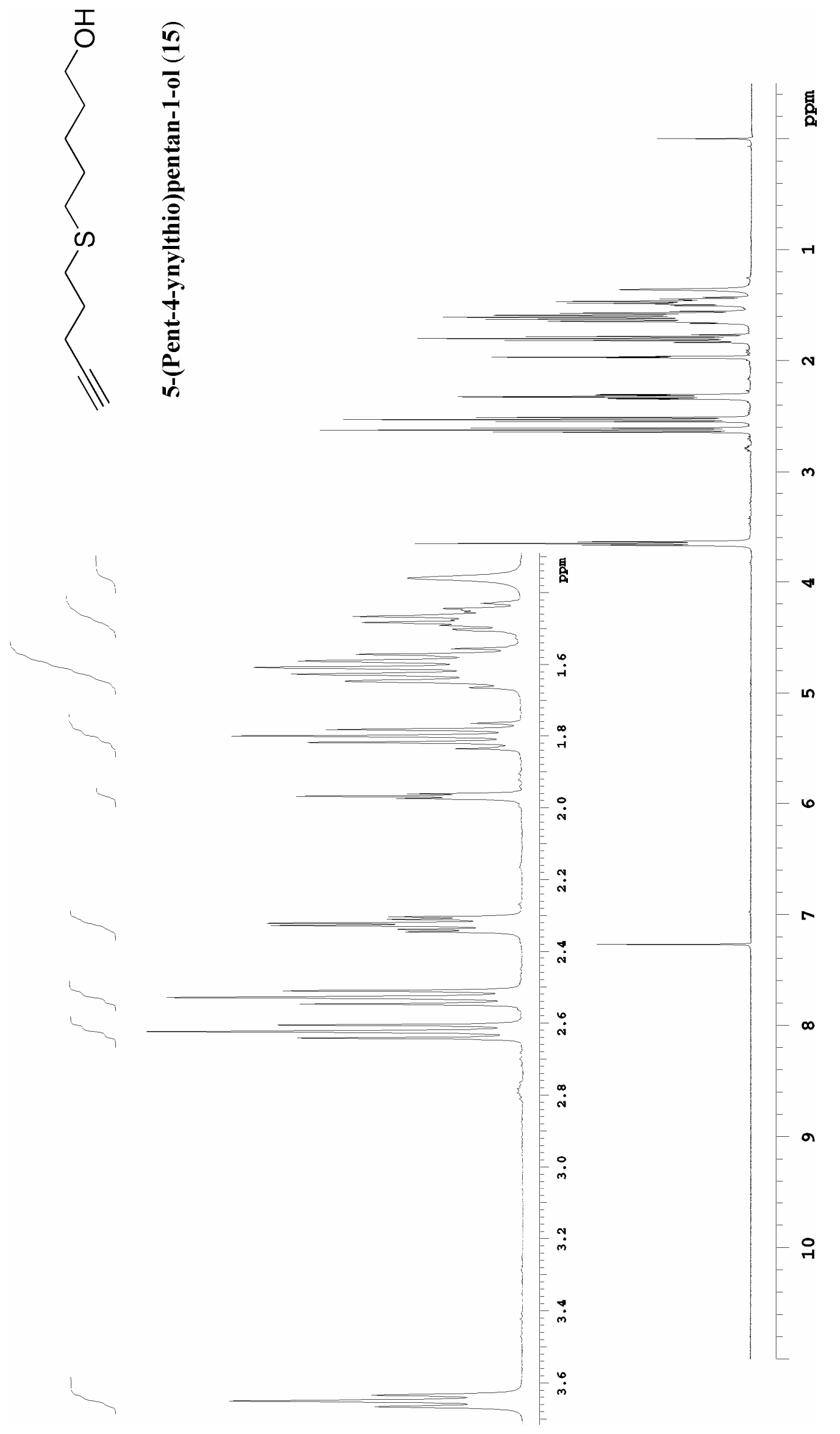




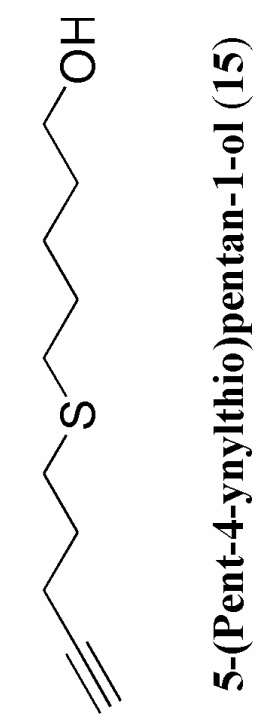

90 n 붕

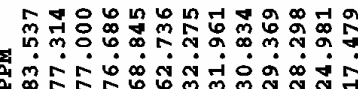

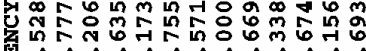

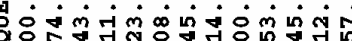

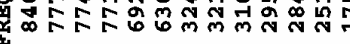

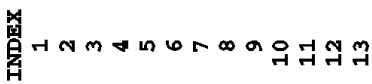

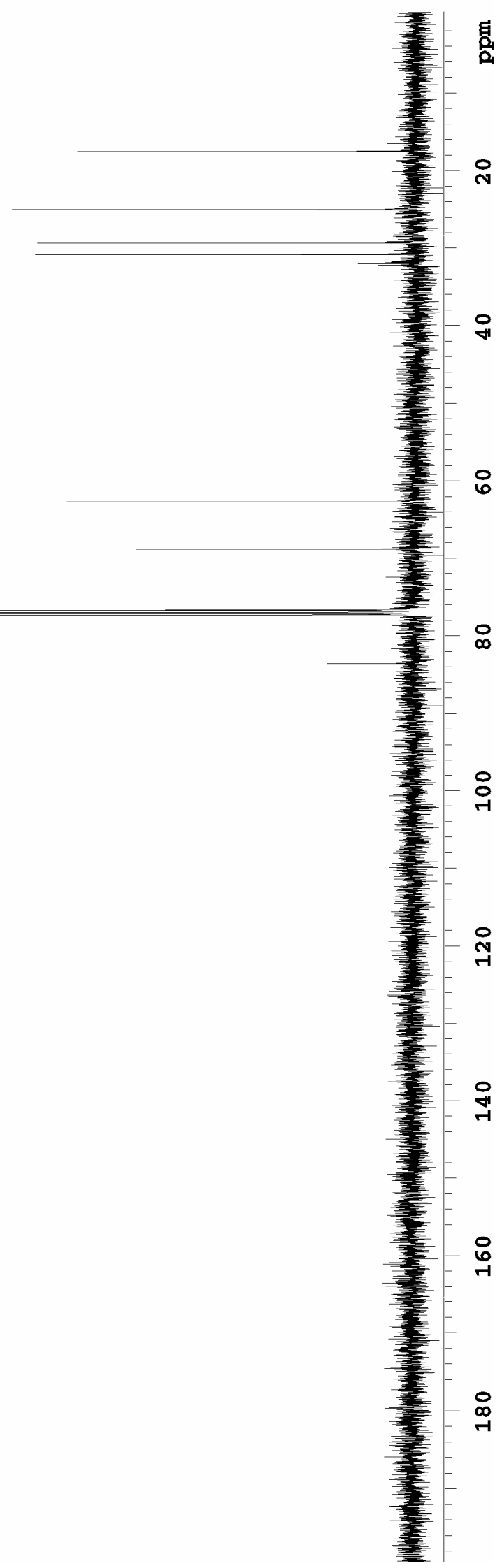



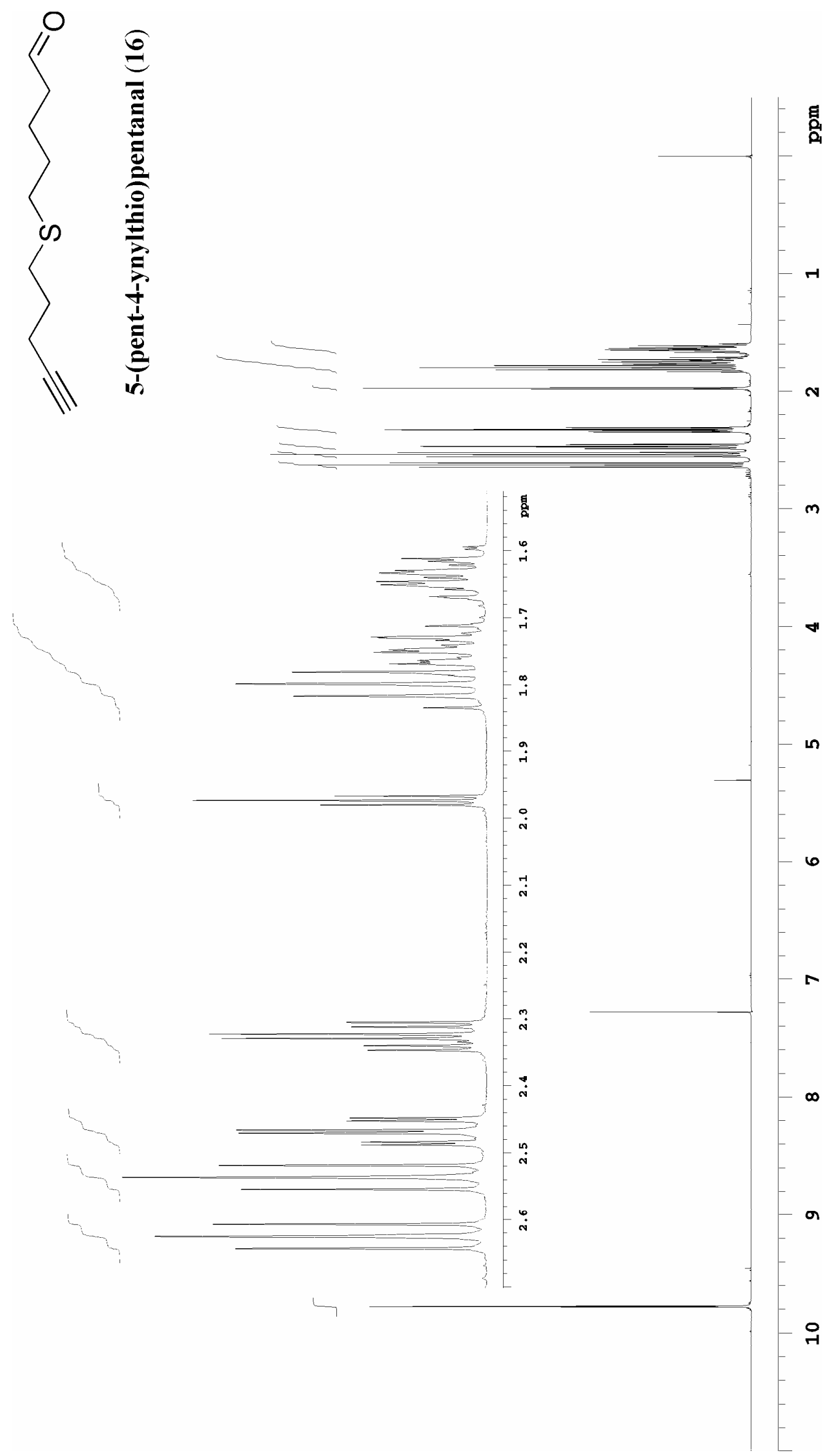


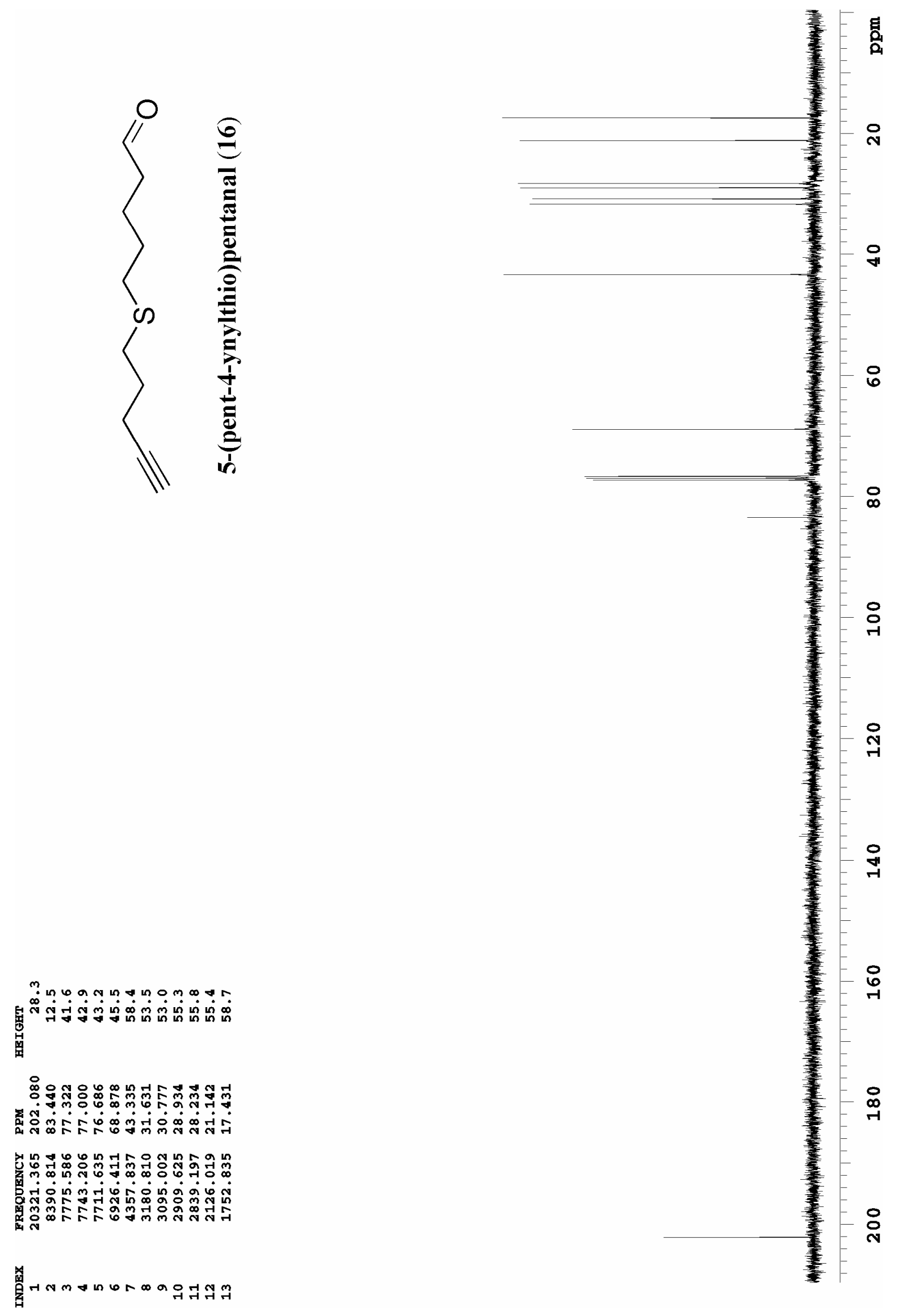




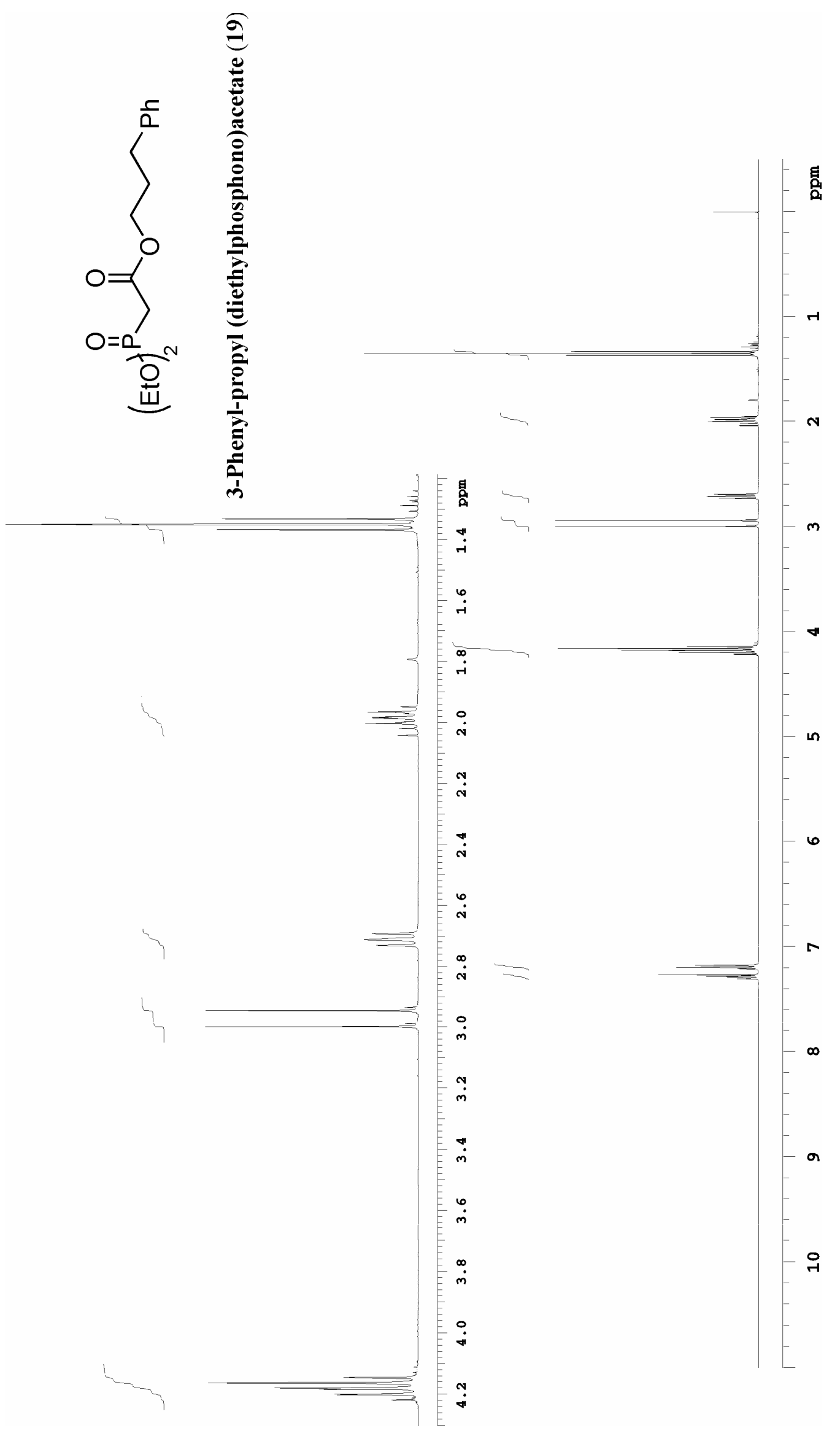




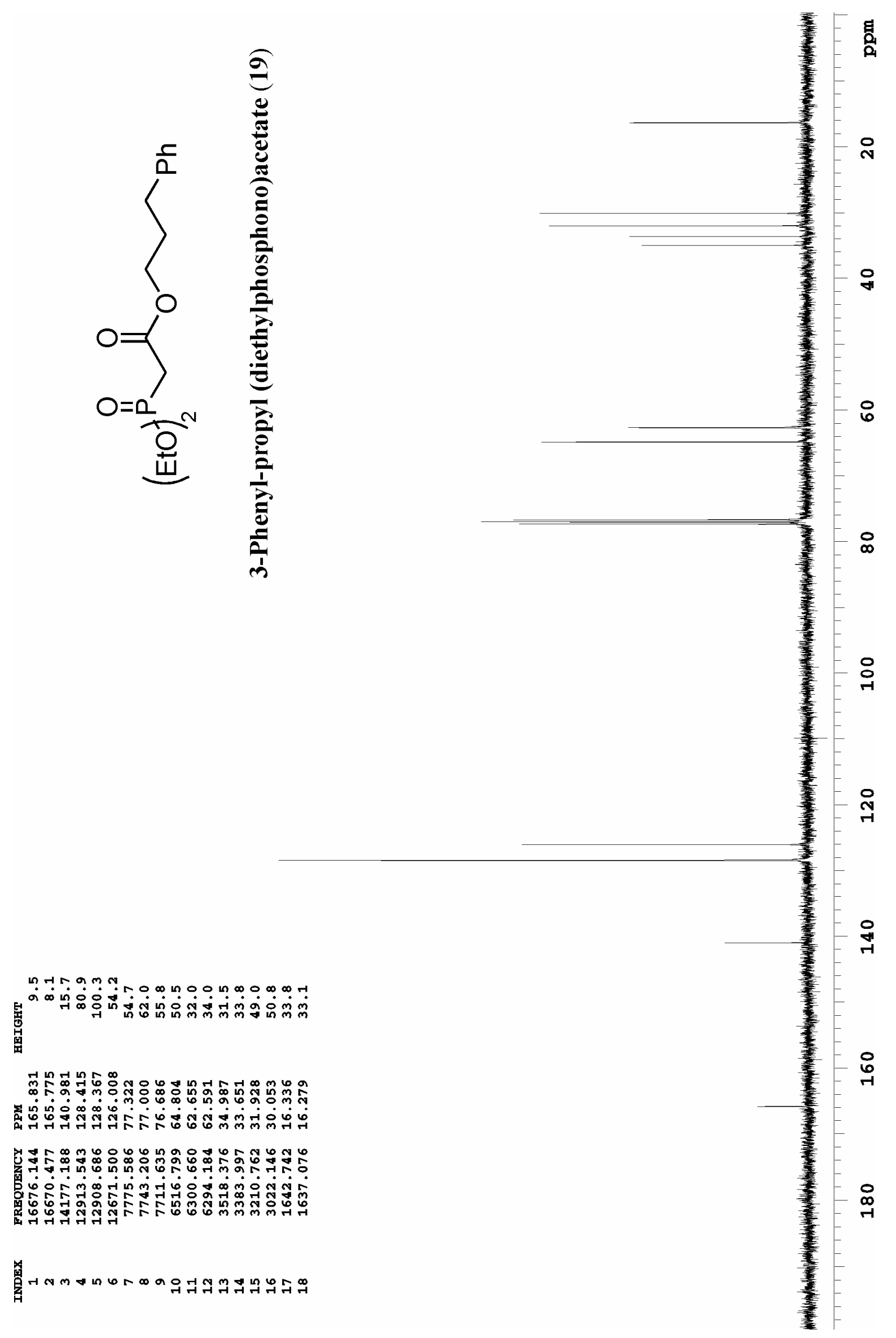

